Florida International University FIU Digital Commons

6-30-2017

\title{
Wireless Positioning and Tracking for Internet of Things in GPS-denied Environments
}

Bekir Sait Ciftler

Florida International University, bcift001@fiu.edu

DOI: 10.25148 /etd.FIDC001959

Follow this and additional works at: https://digitalcommons.fiu.edu/etd

Part of the Systems and Communications Commons

\section{Recommended Citation}

Ciftler, Bekir Sait, "Wireless Positioning and Tracking for Internet of Things in GPS-denied Environments" (2017). FIU Electronic Theses and Dissertations. 3377.

https://digitalcommons.fiu.edu/etd/3377

This work is brought to you for free and open access by the University Graduate School at FIU Digital Commons. It has been accepted for inclusion in FIU Electronic Theses and Dissertations by an authorized administrator of FIU Digital Commons. For more information, please contact dcc@fiu.edu. 


\section{FLORIDA INTERNATIONAL UNIVERSITY \\ Miami, Florida}

\section{WIRELESS POSITIONING AND TRACKING FOR INTERNET OF THINGS IN GPS-DENIED ENVIRONMENTS}

A dissertation submitted in partial fulfillment of the requirements for the degree of DOCTOR OF PHILOSOPHY

in

ELECTRICAL ENGINEERING

by

Bekir Sait Ciftler 


\section{To: Interim Dean Ranu Jung}

College of Engineering and Computing

This dissertation, written by Bekir Sait Ciftler, and entitled Wireless Positioning and Tracking for Internet of Things in GPS-denied Environments, having been approved in respect to style and intellectual content, is referred to you for judgment.

We have read this dissertation and recommend that it be approved.

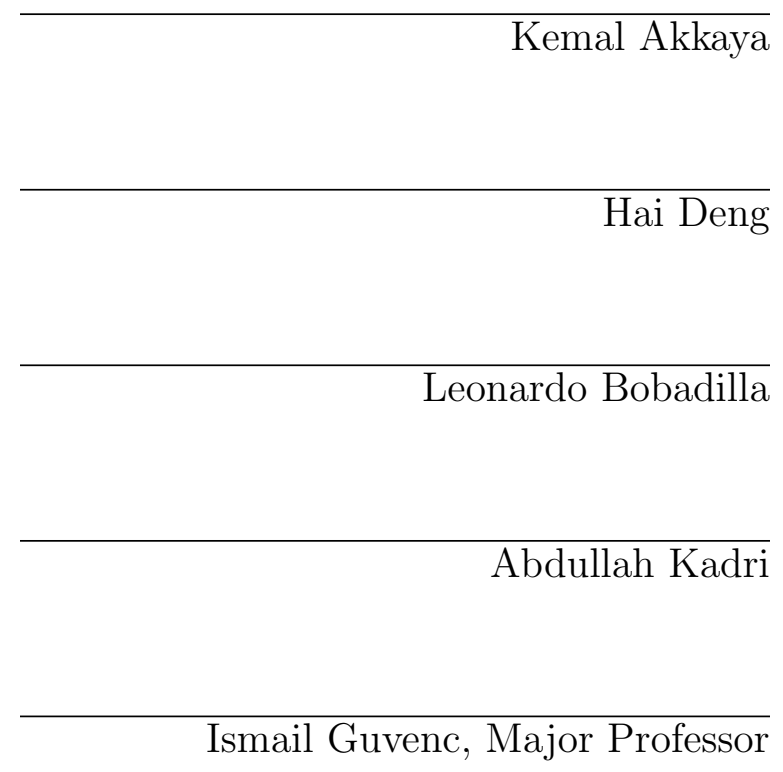

Date of Defense: June 30, 2017

The dissertation of Bekir Sait Ciftler is approved.

Interim Dean Ranu Jung
College of Engineering and Computing

College of Engineering and Computing

Andres G. Gil

Vice President for Research and Economic Development and Dean of the University Graduate School

Florida International University, 2017 
(C) Copyright 2017 by Bekir Sait Ciftler

All rights reserved. 


\section{DEDICATION}

To my beloved wife Fatma... 


\section{ACKNOWLEDGMENTS}

I wish to thank and pay my gratitude to the members of my committee for their insightful comments, encouragement, and generous support. I am extremely thankful and express my deepest gratitude to my major professor, Dr. Ismail Guvenc, for his valuable guidance and immense support for completing this research. He steered me in the right direction during my research and has always been there for help whenever I ran into troubles. His guidance helped me in all the time of research and writing of this dissertation. I intend to follow his example in many respects throughout my career. I would like to thank my fellow lab mates for the stimulating discussions, and for all the fun we have had in the last four years. My special sincere thanks have to go to my parents, Ahmet Necip and Donay Yildiz, for giving me the best of what parents can give. I would like to extend my thanks to my brothers, Mustafa and Faruk, and to my sister, Zeynep, for too many things to mention. Finally, my wholeheartedly thanks go to my beloved wife, Fatma. Her support, encouragement, quiet patience and unwavering love were undeniably the bedrock upon which the past four years of my life have been built. 


\author{
ABSTRACT OF THE DISSERTATION \\ WIRELESS POSITIONING AND TRACKING FOR INTERNET OF THINGS \\ IN GPS-DENIED ENVIRONMENTS \\ by \\ Bekir Sait Ciftler \\ Florida International University, 2017 \\ Miami, Florida \\ Professor Ismail Guvenc, Major Professor
}

Wireless positioning and tracking have long been a critical technology for various applications such as indoor/outdoor navigation, surveillance, tracking of assets and employees, and guided tours, among others. Proliferation of Internet of Things (IoT) devices, the evolution of smart cities, and vulnerabilities of traditional localization technologies to cyber-attacks such as jamming and spoofing of GPS necessitate development of novel radio frequency $(\mathrm{RF})$ localization and tracking technologies that are accurate, energy-efficient, robust, scalable, non-invasive and secure. The main challenges that are considered in this research work are obtaining fundamental limits of localization accuracy using received signal strength (RSS) information with directional antennas, and use of burst and intermittent measurements for localization. In this dissertation, we consider various RSS-based techniques that rely on existing wireless infrastructures to obtain location information of corresponding IoT devices. In the first approach, we present a detailed study on localization accuracy of UHF RF IDentification (RFID) systems considering realistic radiation pattern of directional antennas. Radiation patterns of antennas and antenna arrays may significantly affect RSS in wireless networks. The sensitivity of tag antennas and receiver antennas play a crucial role. In this research, we obtain the fundamental limits of localization accuracy considering radiation patterns and sensitivity of the antennas 
by deriving Cramer-Rao Lower Bounds (CRLBs) using estimation theory techniques. In the second approach, we consider a millimeter Wave (mmWave) system with linear antenna array using beamforming radiation patterns to localize user equipment in an indoor environment. In the third approach, we introduce a tracking and occupancy monitoring system that uses ambient, bursty, and intermittent WiFi probe requests radiated from mobile devices. Burst and intermittent signals are prominent characteristics of IoT devices; using these features, we propose a tracking technique that uses interacting multiple models (IMM) with Kalman filtering. Finally, we tackle the problem of indoor UAV navigation to a wireless source using its Rayleigh fading RSS measurements. We propose a UAV navigation technique based on Q-learning that is a model-free reinforcement learning technique to tackle the variation in the RSS caused by Rayleigh fading. 


\section{TABLE OF CONTENTS}

1. INTRODUCTION . . . . . . . . . . . . . . . . . . . . 1

1.1 What is Localization? . . . . . . . . . . . . . . . . . . . . . . . . . 1

1.2 Localization Techniques . . . . . . . . . . . . . . . . . . . . 2

1.3 Emerging Applications for Localization and Tracking . . . . . . . . . . 3

1.4 Organization and Contributions of this Dissertation . . . . . . . . . 5

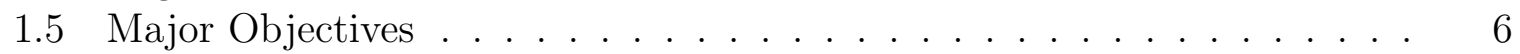

2. LOCALIZATION WITH RFID TECHNOLOGIES $\ldots \ldots \ldots \ldots$

2.1 Literature Review . . . . . . . . . . . . . . . . . . . . . . . . . . . . . . 9

2.2 System Model . . . . . . . . . . . . . . . . . . . . . . . . . . . . . . . . . . 12

$2.2 .1 \quad$ Bistatic RFID Configuration . . . . . . . . . . . . . . . . . . . 13

2.2 .2 Translation to Cartesian Coordinate System . . . . . . . . . . . . . . 15

$2.2 .3 \quad$ Monostatic RFID Configuration . . . . . . . . . . . . . . . . . 15

2.3 Tag / Reader Antenna Sensitivity and Localization Coverage . . . . . . . 16

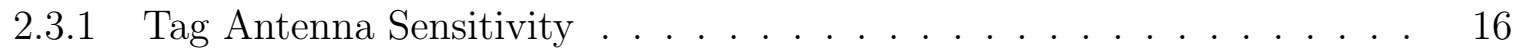

2.3 .2 Reader Antenna Sensitivity . . . . . . . . . . . . . . . . . . . . 18

2.3 .3 Coverage Areas for Localization . . . . . . . . . . . . . . . . . . . 18

2.4 Cramer-Rao Lower Bound and MLE . . . . . . . . . . . . . . . . 20

2.4 .1 Likelihood Function for Unknown RFID Tag Localization . . . . . . . 20

$2.4 .2 \quad$ CRLB Analysis . . . . . . . . . . . . . . . . . . . . . . . . . . . . . . . 21

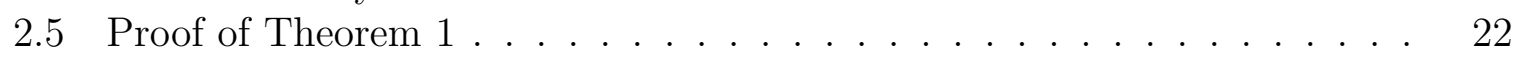

2.6 Example Derivation for CRLB . . . . . . . . . . . . . . . . . . . . . . . . 23

2.6 .1 Maximum Likelihood Estimator . . . . . . . . . . . . . . . . . . . . . . 25

2.7 Numerical Results $\ldots \ldots \ldots \ldots \ldots$

2.7 .1 Localization Coverage . . . . . . . . . . . . . . . . . . . . . . . 27

2.7 .2 Localization Accuracy $\ldots \ldots \ldots$

3. RSS-BASED MMWAVE LOCALIZATION . . . . . . . . . . . 35

3.1 System Model . . . . . . . . . . . . . . . . . . . . . . . . . . . 36

3.2 Sector Level Sweeping . . . . . . . . . . . . . . . . . . . . . . . . . 38

3.3 RSS-Based Localization $\ldots \ldots \ldots$

3.4 Cramer-Rao Lower Bound . . . . . . . . . . . . . . . . . . . . 41

3.5 Derivation of the CRLB . . . . . . . . . . . . . . . . . . . . . 41

3.5 .1 Asymptotic Behavior of CRLB $\ldots \ldots \ldots \ldots$

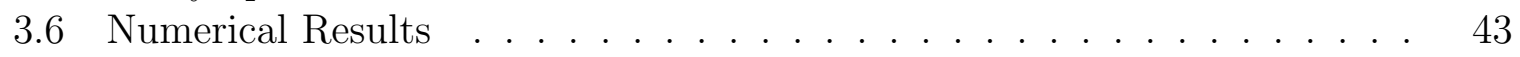

3.7 Conclusion $\ldots \ldots \ldots \ldots \ldots$ 
4. OCCUPANCY COUNTING USING PROBE REQUESTS . . . . . . . . . 49

4.1 Literature Review . . . . . . . . . . . . . . . . . . . . . . 53

4.2 System Model . . . . . . . . . . . . . . . . . . . . . . . . . . . . 54

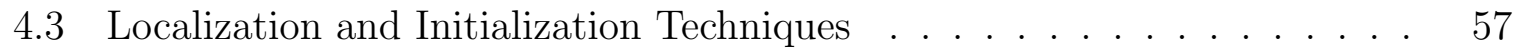

4.3 .1 RSS Available at Three or Less Reference Nodes . . . . . . . . . . . . 58

4.3 .2 RSS Available at Four or More Reference Nodes . . . . . . . . . . . . . 61

4.4 IMM Tracking and Occupancy Counting . . . . . . . . . . . . . . . . . . 63

4.4 .1 Review of a Generic Kalman Filter . . . . . . . . . . . . . . . . . . . . 64

4.4 .2 IMM Based Kalman Tracking of Target Location . . . . . . . . . . . . 65

$4.4 .3 \quad$ Occupancy Counting . . . . . . . . . . . . . . . . . . . . 68

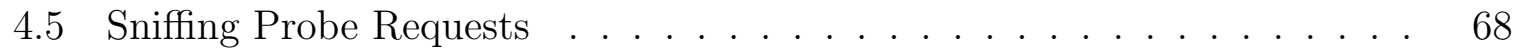

4.6 Numerical Results $\ldots \ldots \ldots$. . . . . . . . . . . . . . . . . . . . . . . . . 70

4.6 .1 Simulation Results . . . . . . . . . . . . . . . . . . . . . . . 70

4.6 .2 Experimental Results . . . . . . . . . . . . . . . . . . . 73

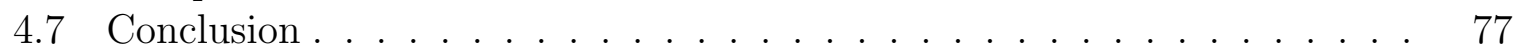

5. INDOOR UAV NAVIGATION . . . . . . . . . . . . . . . . . . . . . 79

$5.1 \quad$ Q-Learning Based Source Tracking . . . . . . . . . . . . . . . . . . . . . 82

5.2 Simulation Results and Conclusion . . . . . . . . . . . . . . . . . . . . . 84

6. CONCLUDING REMARKS \& FUTURE WORK . . . . . . . . . . . . 87

BIBLIOGRAPHY . . . . . . . . . . . . . . . . . . . . . 90

VITA . . . . . . . . . . . . . . . . . . . . . . . . . . 101 


\section{LIST OF TABLES}

TABLE PAGE

$2.1 \quad$ Literature for RFID-based localization systems . . . . . . . . . . . . . . 12

2.2 Passive UHF RFID system parameters. . . . . . . . . . . . . . . . . . . 26

$3.1 \quad$ Simulation parameters. $\ldots \ldots \ldots$. . . . . . . . . . . . . . . . . . 43

4.1 Tracking models under various circumstances. . . . . . . . . . . . . . . . 59

$4.2 \quad$ Simulation parameters of WiFi Probe Request-based tracking. . . . . . . 70

$5.1 \quad$ UAV states with respect to source RSS. . . . . . . . . . . . . . . . . . . 82 


\section{LIST OF FIGURES}

FIGURE

PAGE

\begin{tabular}{|c|c|}
\hline $1.1 \quad$ Occupancy monitoring using various different IoT technologies. Darker \\
\hline colors represent a better occupancy monitoring accuracy, and white \\
\hline colors represent no occupancy monitoring in particular building zone. \\
\hline
\end{tabular}

$2.1 \quad$ Passive RFID localization system with bistatic configuration. In monostatic configuration, reverse link is available only for the RFID antenna establishes forward link. . . . .......... 8

2.2 (a) In the monostatic configuration, the signal transmitted by reader antenna (tilted by angle $\theta_{i}$ ) powers the tag in the forward link. The backscatter link signal, which carries the information of the tag, is received back at the same reader antenna. (b) In the bistatic configuration, the signal transmitted by reader antenna $i$ (tilted by angle $\left.\theta_{i}\right)$ powers the tag in the forward link. The backscatter link signal, which caries the information of the tag, is received at reader antenna $j$

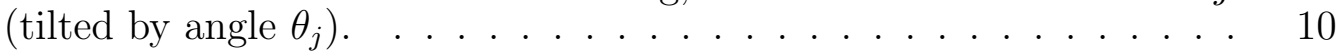

2.3 (a) Maximum achievable RSS at any possible tag location for the system. (b) Localization coverage for monostatic configuration. (c) Localization coverage for bistatic configuration. Areas where $M \geq 2$ are considered to be localizable, and the deployment parameters are $\theta_{i}=\pi / 4$,

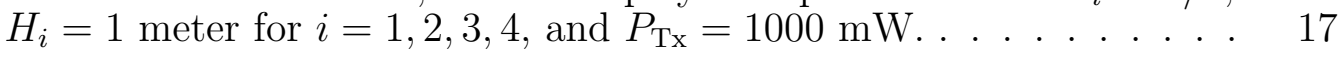

2.4 Localization coverage percentage for $\theta_{i}=\pi / 4, \pi / 3, \pi / 2$, and $i=1,2,3,4$, (a) monostatic and (b) bistatic configurations. . . . . . . . . . . 27

2.5 Average MLE and CRLB RMSE for monostatic and bistatic configurations for $\theta_{i}=\pi / 4, H_{i}=1$ meter, for $i=1, \ldots, 4$. $\ldots . . . .2929$

2.6 Deployment with $\boldsymbol{\theta}_{\boldsymbol{i}}=\boldsymbol{\pi} / \mathbf{4}$ for $i=1,2,3,4$, (a) Median localization RMSE of MLE and CRLB for various transmit powers, (b) CDF' of RMSE of MLE and CRLB with side placement, (c) CDF of RMSE of MLE and CRLB with corner placement. . . . . . . . . . . . 30

2.7 Deployment with $\boldsymbol{\theta}_{\boldsymbol{i}}=\boldsymbol{\pi} / \mathbf{3}$ for $i=1,2,3,4$, (a) Median localization RMSE of MLE and CRLB for various transmit powers, (b) CDF of RMSE of MLE and CRLB with side placement, (c) CDF of RMSE of MLE and CRLB with corner placement. . . . . . . . . . 31

2.8 Deployment with $\boldsymbol{\theta}_{\boldsymbol{i}}=\boldsymbol{\pi} / \mathbf{2}$ for $i=1,2,3,4$, (a) Median localization RMSE of MLE and CRLB for various transmit powers, (b) CDF of RMSE of MLE and CRLB with side placement $\left(P_{\mathrm{Tx}}=3000 \mathrm{~mW}\right)$, (c) CDF of RMSE of MLE and CRLB with corner placement and $\left(P_{\mathrm{Tx}}=3000 \mathrm{~mW}\right) . \ldots \ldots \ldots \ldots \ldots \ldots$

2.9 CDF of RMSE of MLE and CRLB with linear placement of antennas for deployment with (a) $\boldsymbol{\theta}_{\boldsymbol{i}}=\boldsymbol{\pi} / \mathbf{4}$, (b) $\boldsymbol{\theta}_{\boldsymbol{i}}=\boldsymbol{\pi} / \mathbf{3}$, and (c) $\boldsymbol{\theta}_{\boldsymbol{i}}=\boldsymbol{\pi} / \mathbf{2}$ for $i=1,2,3,4\left(P_{\mathrm{Tx}}=3000 \mathrm{~mW}\right) . \cdots \ldots . \ldots . \cdots 33$ 
3.1 An omnidirectional transmitter antenna at UE and $N$-element receiver antenna array at BS as IEEE 802.11ad mmWave communication system. 37

3.2 Azimuthal radiation pattern of linear antenna array and locations of BS

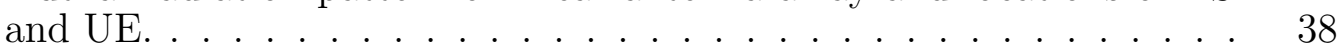

$3.3 \quad$ Sector Level Sweeping. . . . . . . . . . . . . . . . . . . . . . . . . . . . . 39

3.4 (a) Maximum achievable RSS at any possible UE location with 32 antenna elements at transmitter using SLS measurements. (b) CRLB for 32 antenna elements based on SLS measurements. (c) NLSE for 32 antenna elements based on SLS measurements. . . . . . . . . . .

3.5 (a) Median Localization RMSE for various number of antenna elements. (b) 1 meter error bound percentage for various number of antenna elements. . . . . . . . . . . . . . . . . . . . . . . . 45

3.6 CDF of Localization RMSE for (a) $N=8$, (b) $N=16$, and (c) $N=32$. $\quad 46$

4.1 Occupancy counting using probe requests: (a) Capturing burst and intermittent probe requests at multiple WiFi Pineapples; (b) Zone-based real-time occupancy counting. . . . . . . . . . . . . . . 50

$4.2 \quad$ Tracking by using varying number of reference nodes. . . . . . . . . . . 54

4.3 Burst and intermittent probe requests from a mobile device, observed at three different $\mathrm{WiFi}$-PAs within different sampling windows, with

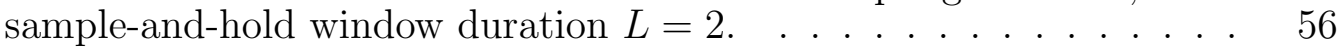

4.4 Tracking scenarios under the different circumstances, (a) initialization with $\mid \mathcal{P}_{k}=1$ and (b) Model-1, (c) initialization with $\mid \mathcal{P}_{k}=2$ and (d) Model-2, and (e) both Initialization with $\left|\mathcal{P}_{k}\right| \geq 3$ and Model-3. . 58

4.5 Map of building floor used in experiments with eight different building zones, as well as the locations of the WiFi sniffers placed across the different zones. . . . . . . . . . . . . . . . . .

4.6 Distribution of passing-by, static and tracked devices by number of probe

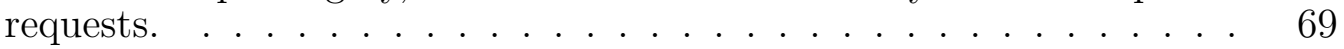

$4.7 \quad$ A sample simulation for a given map (Fig. 4.5p. . . . . . . . . . . . . . . 71

4.8 CDF of mean tracking RMSE for the simulation. . . . . . . . . . . . . . 72

4.9 Zone-level localization performance. . . . . . . . . . . . . . . . . . 73

\begin{tabular}{|cc|c|}
\hline 4.10 & Simulation and experiment performance comparison of zone-level local- \\
\hline ization with and without IMM. . . . . . . . . . . . . . 74
\end{tabular} 
4.11 Total number of occupants on a weekday for sample-and-hold window lengths $L=1$ and $L=15$. Scenario with $N_{\min }=4$ necessitates at least four reference nodes to localize a target device, while $N_{\min }=1$ can localize a target device within a building zone using a single reference

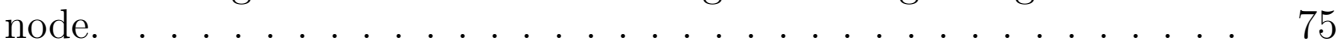

4.12 Total number of occupants within the individual zones given in Fig. 4.5 throughout a weekday. . . . . . . . . . . . . . . 76

\begin{tabular}{|lll|l|l|}
\hline 4.13 Total number of occupants within individual zones given in Fig. & 4.5 & at
\end{tabular} peak hours. . . . . . . . . . . . . . . . . . . 77

4.14 The distribution of the occupancy hours for the individual occupants. $\quad 78$

$5.1 \quad$ Navigation to a Rayleigh fading wireless source. . . . . . . . . . . . . . . 81

5.2 Rayleigh fading RSS for three UAV velocities. . . . . . . . . . . . . . . . 81

5.3 Map of FIU Engineering Center 3rd floor. . . . . . . . . . . . . . . . . . 84

5.4 Convergence time of (a) Q-learning (varying learning rate); (b) Q-learning (fixed learning rate); (c) RL (single state). . . . . . . . . . 85 


\section{CHAPTER 1 \\ INTRODUCTION}

Wireless localization has long been a critical technology for various applications such as indoor/outdoor navigation, surveillance, tracking of assets and employees, and guided tours, among others. Wireless localization based on radio frequency (RF) signal observations have therefore been studied extensively in the existing literature $[1-3]$.

The proliferation of Internet of Things (IoT) devices, evolution of smart cities, and vulnerabilities of traditional localization technologies to cyber-attacks such as jamming and spoofing of GPS necessitate development of novel RF localization technologies that are accurate, energy-efficient, robust, scalable, non-invasive and secure. The main goals of this dissertation are to address several of these challenges considering applications in emerging IoT networks, smart buildings, and GPS-denied environments.

In this dissertation, we first introduce the concept of localization and tracking. Then we review the trends in IoT technologies and smart cities to have a better context on required localization techniques in such environments. We also discuss disadvantages of GPS, and why we strongly need alternative localization techniques that can work effectively in GPS-denied environments.

\subsection{What is Localization?}

Localization is the task of estimating a target node's location using the measurements obtained from the target node at various reference nodes. These reference nodes are commonly referred as anchor nodes, and their locations are assumed known a priori. There are four general types of measurements that can be used in RF localization 
systems [3,4]: received signal strength (RSS), time-of-arrival (TOA), time-differenceof-arrival (TDOA), and angle-of-arrival (AOA).

\subsection{Localization Techniques}

As the name suggests, TOA is the propagation time of the signal between the target node and the reference nodes. Assuming known positions of base nodes and a coplanar scenario, three base nodes and three measurements of distances (TOA) are required to localize a target node. Potential target location will lie on a circle with radius estimated by TOA. Final target location will be the point that is the intersection of the circles.

TDOA is the difference of arrival times between reference nodes. Similar to TOA estimation, this method assumes that the locations of base nodes are known. Final target location will be the point of intersection of two hyperbolas, which are formed using two TDOA measurements with respect to the reference node.

The AOA typically requires directional antennas to detect the respective angle of the target node to reference nodes. Potential target location will lie on a line whose direction is determined by peak incoming energy signal using antenna array. Final target location will be a point that passed through the intersection of two lines whose directions are determined by peak incoming energy signals using antenna arrays at two reference nodes.

RSS is based on signal power which is received either at the target node or reference

nodes. RSS-based localization does not require additional or sophisticated software as others. It employs received power level to extract ranging information to locate the target node based on reference nodes' a priori known positions. 


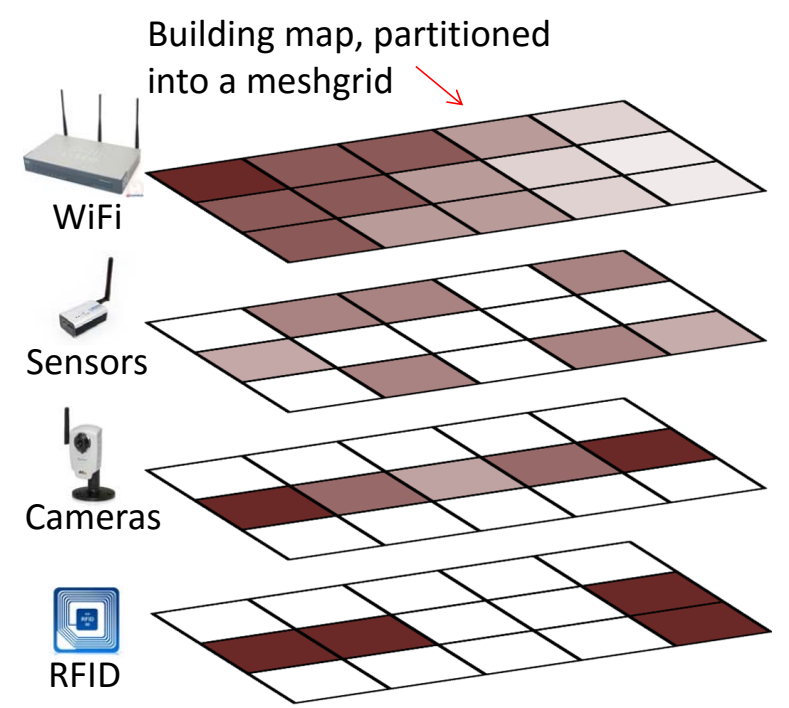

Figure 1.1: Occupancy monitoring using various different IoT technologies. Darker colors represent a better occupancy monitoring accuracy, and white colors represent no occupancy monitoring in particular building zone.

\subsection{Emerging Applications for Localization and Tracking}

Internet of Things: According to Cisco, during 2008, the number of things connected to the Internet surpassed the number of people on earth. In 2020, 50 to 200 billion devices are projected to be connected to the Internet [5]. Such proliferation of IoT devices including smartphones, sensors, cameras, and RFIDs, bluetooth devices, will introduce a critical need to localize and track them effectively, and will necessitate development of energy efficient, accurate, and scalable localization technologies. Connectivity aspects of IoT devices would allow use of cloud computing. Cloud is a great enabler for scaling of the application and innovative approaches with big data.In this dissertation, one of our focus areas is RFID-based wireless localization for IoT technologies. Since passive RFID tags do not operate with any battery power (energy is harvested from RFID antenna that powers the RFID tag), and since they have low deployment cost, they are ideal candidates for localization in IoT applications. 
Smart Cities and Smart Buildings: In addition to IoT, Smart City is another emerging paradigm, where main goals include better use of public resources, increasing the quality of service offered to citizens, and reducing operational costs of public administrators [6]. RF localization is one of the major enablers for future smart cities, and it can support a plethora of services, such as building health inspection, waste management, noise/air quality monitoring, traffic congestion control, city energy consumption reduction, smart parking/lighting, and automation of smart buildings $[7 \sqrt{9}$. Buildings are among the largest consumers of electricity in the United States: they account for $40 \%$ of primary energy consumption and $72 \%$ of electricity consumption [6]. Occupancy tracking that relies on RF localization can help in achieving significant energy savings in smart buildings such as by dynamically scheduling HVAC activity based on real-time building occupancy levels at different areas [10]. Effective occupancy monitoring in smart buildings will be another major application area in this dissertation, for which we will rely on processing of non-invasive RF localization techniques using ambient wireless signals radiating from smart-phones of building occupants. An example use case that considers multiple wireless technologies for occupancy tracking in smart buildings is shown in Fig. 1.1.

GPS Denied Environments. While GPS has been extensively used for outdoor navigation during the past decades, it does not work well in urban canyons (such as in downtown areas) and indoor environments due to weak penetration of GPS signals to such environments. More importantly, GPS has been recently more vulnerable to spoofing and jamming attacks. Due to such vulnerabilities of GPS against cyber attacks, department of defense in the United States has been looking into developing of localization technologies that can operate effectively in the absence of GPS location information. Study of wireless localization technologies in GPS-denied environments, 
such as in indoor environments or under jamming attacks, will be another important research component of this dissertation.

Not only non-line of sight situations but also jamming and spoofing attacks are a problem for GPS. GPS signals are not encrypted, thus, it is very easy to jam or spoof them. GPS is also susceptible to record-and-replay attacks if it is not secured with other countermeasures. There are several measures existing in our devices though they are still unreliable since most of their techniques are known and obsolete. It is relatively easier after one develops a GPS emulator algorithm and uses a softwaredefined radio to implement [2]. As a result, indoor and urban environments are GPSdenied areas which require different solutions for localization and tracking problems.

A major application of wireless localization in GPS-denied environments is locationbased services in emergency and public safety applications. For example, in the case of a 911 call, FCC requires that 80 percent of the calls should be located within 50 meters by 2021. Police and firefighters also require seamless localization and tracking for public safety applications. In case of a fire in a large building, it becomes difficult to navigate in the building to save a victim or to find an exit. Accurate localization in such GPS-denied indoor environments is still an open research challenge, which can affect the lives of first responders and victims.

\subsection{Organization and Contributions of this Dissertation}

In previous subsection, we pointed out applications enabled with indoor localization systems based on IoT and why we need them. This leads us to a research question, as stated below

Starting with limited prior information, is it possible to detect, locate and track the user equipped with Internet of Things devices? 


\subsection{Major Objectives}

In this project, we developed accurate RSS-based localization and tracking techniques in GPS-denied environments, with applications to IoT technologies and smart cities. The performance of prior localization algorithms in such scenarios were mediocre and far from achievable performance limits, and our goal was increasing the localization accuracy as close as possible to achievable limits using estimation theory techniques and machine learning methods. Based on the fundamental research problems that are considered, the objectives of this research can be categorized into three main thrusts as follows:

- Localization with Directional RF Transmissions: Realistic 3D path-loss and radiation models will be used to study fundamental lower bounds on the localization accuracy for RFID and mmWave technologies with applications to IoT scenarios. Both these technologies are recently becoming more widely deployed, and they both utilize directional transmissions. Comparison of different localization algorithms for such directional transmissions will be made with respect to fundamental lower bounds such as the CRLB.

- Ubiquitous Localization and Tracking in Indoor Environments: Using the tools of estimation theory, localization and tracking of the target nodes will be ubiquitously available in a heterogeneous environment where number of available anchor nodes and RF propagation parameters are dynamically varying. Heuristic techniques and nonlinear least square estimators will be implemented to localize the target node in smart building environments. A interacting multiple Kalman Filters are developed to track the target node for occupancy tracking. Machine-learning techniques will be used to learn optimum propagation and occupancy parameters and maximize localization accuracy. 


\section{CHAPTER 2}

\section{LOCALIZATION WITH RFID TECHNOLOGIES}

Radio Frequency IDentification (RFID) is a promising technology for the proliferation of Internet of Things (IoT) applications, and it can be used to detect and identify the items in the proximity 11 15. Due to their cost effective, durable, and energy efficient operation [16], RFID technology has been used in wide range of applications such as asset management [17], access control [18], public safety [19], localization [20], and tracking [21]. Among these, enabling high accuracy localization for massively deployed IoT devices carries critical importance for a diverse set of IoT applications 22].

Localization using radio frequency $(\mathrm{RF})$ signals has been actively researched in the literature over the past decades $[23[26]$. Outdoor localization is mostly handled with Global Positioning System (GPS) technology whereas indoor localization

requires alternative approaches since GPS needs a line-of-sight connection between user equipment and satellites. Moreover, massive deployment of IoT devices necessitates energy and cost efficient localization methods for prolonged durations. The RFID technology hence becomes a promising alternative for cost-effective, energy efficient indoor identification and localization for massively deployed IoT.

An Ultra High Frequency (UHF) RFID communication is fundamentally different from the conventional RF communication since it has two distinct links: the forward (power-up) and the reverse (backscatter) link. The forward link powers the passive RFID tags and the reverse link carries the information of tags. Ability to power-up tags in the forward link enables battery-less operation of RFID tags [27], which is a major advantage of RFID systems for low-power IoT applications. In general, there are two configurations for UHF RFID systems: 1) monostatic configuration, and 2) bistatic configuration. In the monostatic configuration, a single reader antenna trans- 


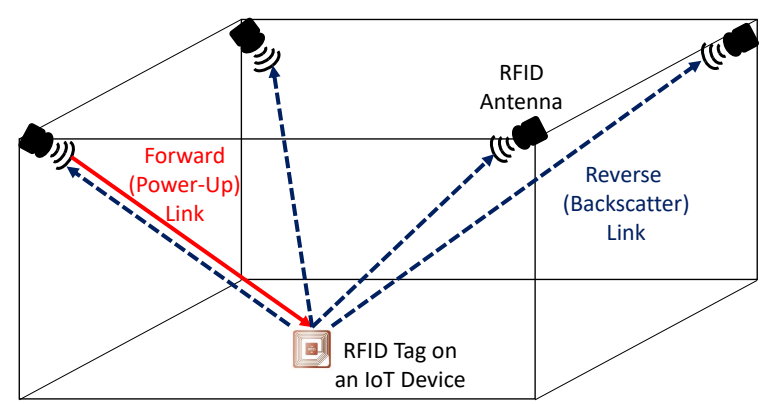

Figure 2.1: Passive RFID localization system with bistatic configuration. In monostatic configuration, reverse link is available only for the RFID antenna establishes forward link.

mits the continuous wave, which powers up the passive tag, and subsequently receives the backscattered information signal from the tag. In the bistatic configuration the transmission and reception are handled by different reader antennas as shown in Fig. 2.1. These antennas might be co-located (i.e., at same location, closely spaced) or dislocated (at separate locations). A particular challenge with both configuration is that complex, directional, and three dimensional RFID propagation models need to be explicitly taken into account to accurately characterize the real-world forward/backward propagation channels.

In this paper, we use sophisticated and realistic 3D path-loss and radiation models to study fundamental lower bounds on the localization accuracy of Received Signal Strength (RSS) based UHF RFID localization systems for both monostatic and bistatic configurations. The main contributions of this work are as follows: 1) CramerRao Lower Bound (CRLB) on the localization accuracy are derived in closed-form considering an enhanced RSS model, using the directional and $3 D$ radiation pattern from UHF RFID reader antennas, and the concept of localization coverage; 2) Tag and reader sensitivity is incorporated into analytic derivations both for monostatic and bistatic scenarios, to derive localizability and localization coverage metrics; 3) Extensive computer simulations are carried out to compare the localization accuracy 
of the maximum likelihood (ML) technique with the CRLBs, considering directional radiation patterns and using different configurations for RFID reader antennas.

Our analysis and simulation results show that for certain scenarios, using bistatic antenna configuration as in Fig. 2.1 may increase the average localization coverage by $38 \%$ when compared to monostatic RFID configuration. Another important parameter in the antenna configurations is the elevation angle $\theta$. Especially with lower transmit powers, it affects the localization coverage and accuracy. Corner placement of antennas for $\theta=\pi / 4$ with $1000 \mathrm{~mW}$ gives $29 \%$ localization coverage, while $\theta=\pi / 3$ and $\theta=\pi / 2$ results in $78 \%$. Our results for the specific RFID configuration show that it is possible to locate a tag within 1 meter error with a probability of 0.76 with corner placement of antennas, whereas this probability drastically reduces to 0.53 when side placement is used for $\theta=\pi / 4$ with bistatic configuration.

The rest of this paper is organized as follows. Literature review for RSS-based localization in passive UHF RFID systems is provided in Section 2.1. In Section 4.2. the system model is described in detail which involves a 3D radio propagation model for RFID systems. The concept of localizability is defined, as well as localization coverage percentage in Section 2.3. Section 2.4 derives the CRLBs and the Maximum Likelihood Estimator (MLE) based on the likelihood function for an RFID tag's location for the considered RFID scenario. Numerical results are provided in Section 2.7. and concluding remarks are given in Section 4.7 .

\subsection{Literature Review}

Although there are several studies in the literature that investigate RSS-based localization with RFID technology [28 32], fundamental lower bounds on RFID-based wireless localization are relatively unexplored. In [33], authors used a mobile robot with RFID reader antennas to generate map of an indoor environment with RFID 


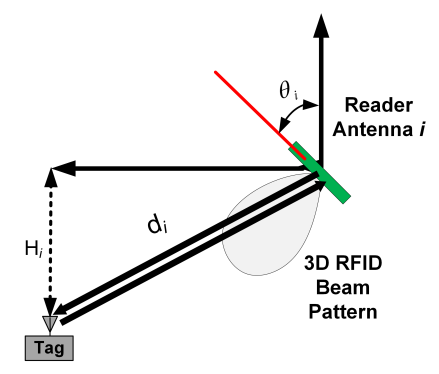

(a)

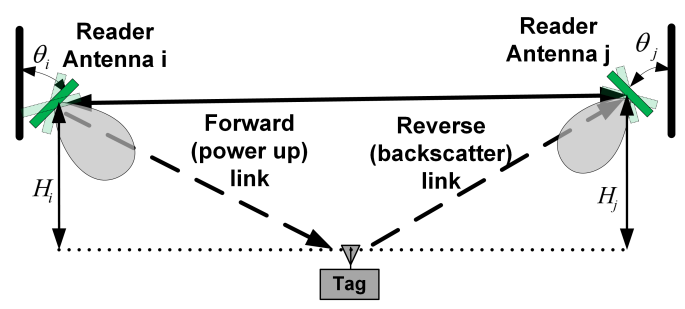

(b)

Figure 2.2: (a) In the monostatic configuration, the signal transmitted by reader antenna (tilted by angle $\theta_{i}$ ) powers the tag in the forward link. The backscatter link signal, which carries the information of the tag, is received back at the same reader antenna. (b) In the bistatic configuration, the signal transmitted by reader antenna $i$ (tilted by angle $\theta_{i}$ ) powers the tag in the forward link. The backscatter link signal, which caries the information of the tag, is received at reader antenna $j$ (tilted by angle $\theta_{j}$ ).

tags on the walls. After the mapping phase, the robot may locate itself inside the building based on the closest tag information. In the LANDMARC localization technique introduced in [34], reference RFID tags are used for implementing RSS-based indoor localization method, where fixed-location reference tags with known locations are used to localize the tags. In [35], authors improve LANDMARC approach to tackle with multipath effects and RF interference. A probabilistic RFID map-based technique with Kalman filtering is used to enhance the location estimation of the RFID tags in [36]. Another approach to localize the RFID tags is studied in [37], which uses the phase difference information of backscattered signal of the RFID tags. In [38], authors consider a multipath environment to derive the CRLBs on the position error of an RFID based wireless localization system. Geometry of the deterministic multipath components and the interfering diffuse multipath components are considered in the backscatter channel model.

Typically a simple path-loss model is used for RFID propagation models in the existing literature [34, 39, 40], which employs free-space path-loss signal strength model. These models are not capable of accurately capturing the radiation pattern of RFID 
reader antennas since they are highly directional. There are also several experimental studies in the literature related to RSS-based UHF RFID localization systems. In [39], an experimentation with passive UHF RFID system is conducted to investigate the relationship between RSS and distance. Recently in [40], CRLB of RSS-based localization are derived considering a frequency dependent path-loss propagation model, where the model explicitly depends on the transmit power level and the transmission frequency. Accuracy of several localization techniques are compared to CRLB with given path-loss model via simulations and experiments. In [41, authors used $k$-Nearest Neighbor $(\mathrm{kNN})$ algorithm to estimate the location of the target tag from RSS information. An experiment involving four antennas and seventy tags is conducted, which resembles to the simulation scenario in our manuscript. It is shown that power control techniques may significantly improve localization accuracy.

Effects of multipath propagation and signal scattering are considered in 42 for passive UHF RFID localization, using MLE and linear least square techniques. A localization algorithm using the differences of RSS values from various tags under same conditions is also proposed. Its performance, which is shown to outperform the kNN algorithm used in LANDMARC [34]. A two-parameter path-loss model for UHF RFID systems is constructed in [43], which shows that the RSS of RFID systems are slightly more stable than WiFi RSS values, and this yields more precise location estimates for RFID RSS-based localization. A summary of the existing RFID-based localization papers in the literature categorized based on the specific localization techniques that are used is provided in Table 2.1.

In our earlier work [44], we have studied the bounds on RFID localization for monostatic RFID configuration. In this study, our additional contributions include: 1) use of bistatic antenna configuration and different antenna placement which provides a more generalized framework, 2) use of an enhanced RSS model with lognormal 
Table 2.1: Literature for RFID-based localization systems

\begin{tabular}{|c|c|}
\hline Category & References \\
\hline "Time-of-Flight & $|28| \overline{30}$ \\
\hline Number of readings & 31 \\
\hline Fingerprinting & $33 \quad 36$ \\
\hline Phase difference & $37, \overline{38}$ \\
\hline RSS Ranging & $32,34,3943,45$ \\
\hline
\end{tabular}

distributed noise which yields different CRLB formulations, 3) incorporation of reader antenna and tag sensitivity into theoretical analysis, 4) study of localization coverage for RFID tags, outside of which they can not be localized with a reasonable accuracy, and 5) extensive new simulations to study the effects of various parameters and configurations.

\subsection{System Model}

In the rest of this paper, we consider the RFID localization scenario as shown in Fig. 2.2. In particular, Fig. 2.2(a) illustrates a monostatic antenna configuration, where the reader antenna is both the transmitter and the receiver. On the other hand, the bistatic antenna configuration is shown in Fig. 2.2(b), where one antenna transmits the power-up signal for RFID tag, and the other antenna receives the backscattered signal from the tag. We will consider the more general case of bistatic antenna configuration, and study the monostatic configuration as a special case. For the considered scenario, let $N$ RFID reader antennas be mounted on the walls, located at a height of $z_{i}$ meters from the ground for the $i$ th antenna. As shown in Fig. 2.2(b), RFID reader antennas $i$ and $j$ (which are the forward and reverse antennas, respectively) are tilted by an angle $\theta_{i}$ and $\theta_{j}$, respectively, with reference to the 
azimuth plane. The goal is to localize an RFID tag, which is located at a distance $H_{i}$ below a reader antenna $i$.

\subsubsection{Bistatic RFID Configuration}

The total backscattered received power $P_{i j}$ at a bistatic configuration of reader antenna $i$ and antenna $j$, which are located at $\left(x_{i}^{\prime}, y_{i}^{\prime}, z_{i}^{\prime}\right)$ and $\left(x_{j}^{\prime}, y_{j}^{\prime}, z_{j}^{\prime}\right)$, respectively while the position of the tag is $\left(x_{0}, y_{0}, z_{0}\right)$, is given by [46]:

$$
\widetilde{P}_{i j}\left(x_{0}, y_{0}, z_{0}\right)=\tau \mu_{\mathrm{T}} \rho_{\mathrm{L}} P_{\mathrm{TX}} G_{\mathrm{T}}^{2}\left|G_{\mathrm{R}}^{i} G_{\mathrm{R}}^{j} L\left(d_{i}\right) L\left(d_{j}\right)\right|\left|h_{i} h_{j} \Gamma\right|^{2},
$$

which can be written in logarithmic scale as

$$
\begin{gathered}
P_{i j}\left(x_{0}, y_{0}, z_{0}\right)[\mathrm{dBm}]=20 \log _{10}\left(\tau \mu_{\mathrm{T}} \rho_{\mathrm{L}} P_{\mathrm{Tx}} G_{\mathrm{T}}^{2}\left|h_{i} h_{j} \Gamma\right|^{2}\right) \\
+20 \log _{10}\left(G_{\mathrm{R}}^{i}\right)+20 \log _{10}\left(G_{\mathrm{R}}^{j}\right) \\
+20 \log _{10}\left(L\left(d_{i}\right)\right)+20 \log _{10}\left(L\left(d_{j}\right)\right),
\end{gathered}
$$

$\forall i, j \in\{1, \ldots, N\}$ where $\tau$ is a coefficient that quantifies the specific data encoding modulation details that can be calculated using power density distribution of the tag's signal.

According to the EPCglobal C1G2 specifications [47], any tag in the interrogation zone of the reader can send back its information by reflecting the incoming continuous

wave. The power transfer efficiency $\mu_{T} \in[0,1]$ in $(2.2)$ quantifies how well the tag is impedance-matched to the antenna. Polarization loss factor $\rho_{\mathrm{L}}$ captures the loss due to the mismatch between the polarization of a transmitter antenna and a receiver antenna. The effective isotropic radiated power (EIRP) of the RFID reader antenna is shown as $P_{\mathrm{Tx}}$, while $G_{\mathrm{R}}^{i}$ and $G_{\mathrm{T}}$ are the gain of the RFID reader antenna $i$ and tag antenna, respectively, and $L\left(d_{i}\right)$ is the channel pathloss defined by:

$$
L\left(d_{i}\right)=\left(\frac{\lambda}{4 \pi d_{i}}\right)^{2},
$$


where $\lambda$ is the wavelength of the signal, and $d_{i}$ is the distance between the tag and the reader antenna $i$. The transmit power limit of RFID reader antennas, which is critical for coverage of the reader, is $2 \mathrm{~W}$ in effective radiated power (ERP) as stated in EPC Gen2 protocol for UHF RFID systems. This makes the EIRP limit for RFID readers $35.15 \mathrm{dBm}$, which is larger than the highest transmit power of $3 \mathrm{~W}$ that was used in our simulations 47,48.

The forward-link and backscatter-link channels are represented with $\left|h_{i}\right|^{2}$ and $\left|h_{j}\right|^{2}$. The parameter $\Gamma$ in $(2.2)$ is the differential reflection coefficient of the tag which is a function of the tag antenna gains $G_{\mathrm{T}}$, the radar cross section RCS denoted by $\sigma_{\mathrm{RCS}}$, and the communication wavelength $\lambda$ as follows $46,48,49$ :

$$
|\Gamma|^{2}=\frac{4 \pi}{\lambda^{2}\left|G_{\mathrm{T}}\right|^{2}} \sigma_{\mathrm{RCS}}
$$

In passive UHF RFID applications, the goal is to maximize RCS, which characterizes the scattered power, while still absorbing sufficient power to operate the chip of the tag. In our study, we have utilized statistical models for RCS and $\Gamma$ which we obtained from $43,48,50$.

Assuming a scenario as illustrated in Fig. 2.2, and adopting the expression provided by [46], a modified directional gain of a patch antenna for a 3D propagation environment can be expressed as follows:

$$
\begin{aligned}
G_{\mathrm{R}}^{i}\left(\alpha_{i}, \phi_{i}\right) & =3.136\left[\tan \left(\alpha_{i}\right) \sin \left(0.5 \pi \cos \left(\alpha_{i}\right)\right)\right. \\
& \left.\times \cos \left(0.5 \pi \sin \left(\alpha_{i}\right) \sin \left(\phi_{i}\right)\right)\right]^{2},
\end{aligned}
$$

where $\alpha_{i}=\theta_{i}-\arcsin \left(\frac{H_{i}}{d_{i}}\right)$, with $\theta_{i}$ and $\phi_{i}$ being the elevation and azimuthal angles of the patch antenna $i$, respectively. The parameter $H_{i}$ in 2.5$)$ is the difference between height of the reader antenna and the height of tag. 


\subsubsection{Translation to Cartesian Coordinate System}

Location of a tag with respect to reader antenna is defined with relative elevation and azimuthal angles, and distance between tag and reader antenna. On the other hand, derivation of CRLB requires translation from polar coordinate system to the Cartesian coordinate system. Gain of patch antenna is defined in (2.5), which can be represented in the Cartesian coordinate system as

$$
\begin{aligned}
& G_{\mathrm{R}}^{i}\left(x_{i}, y_{i}, z_{i}, x_{0}, y_{0}, z_{0}, \theta_{i}, \phi_{i}\right) \\
& =3.136 \times\left(\frac{\tan \theta_{i}-\frac{z_{i}-z_{0}}{d_{i}}}{1-\frac{z_{i}-z_{0}}{d_{i}} \tan \theta_{i}}\right)^{2} \\
& \times \sin ^{2}\left(\frac{\pi}{2}\left(\frac{l_{i}}{d_{i}} \cos \theta_{i}+\frac{z_{i}-z_{0}}{d_{i}} \sin \theta_{i}\right)\right) \\
& \times \cos ^{2}\left(\frac{\pi}{2}\left(\frac{l_{i}}{d_{i}} \sin \theta_{i}-\frac{z_{i}-z_{0}}{d_{i}} \cos \theta_{i}\right)\right. \\
& \left.\times\left(\frac{\left(x_{i}-x_{0}\right) \cos \phi_{i}+\left(y_{i}-y_{0}\right) \sin \phi_{i}}{l_{i}}\right)\right),
\end{aligned}
$$

where $\left(x_{i}, y_{i}, z_{i}\right)$ is coordinate of the antenna- $i$, and $\left(x_{0}, y_{0}, z_{0}\right)$ is the location of tag. The distance between tag and antenna- $i$ is defined with

$$
d_{i}=\sqrt{\left(x_{0}-x_{i}\right)^{2}+\left(y_{0}-y_{i}\right)^{2}+\left(z_{0}-z_{i}\right)^{2}} \text {, }
$$

while its projection on the $x y$-plane is given with

$$
l_{i}=\sqrt{\left(x_{0}-x_{i}\right)^{2}+\left(y_{0}-y_{i}\right)^{2}} .
$$

\subsubsection{Monostatic RFID Configuration}

As in Fig. 2.2(a), monostatic RFID is a special case of bistatic RFID configuration, there the transmitter and receiver antenna are identical. This makes $G_{R}^{i}$ and $G_{R}^{j}$ equal $(i=j)$. Therefore, the received power in $\mathrm{dBm}$ at the reader antenna with 
monostatic configuration simplifies to:

$$
\begin{aligned}
P_{i i}\left(x_{0}, y_{0}, z_{0}\right) & =20 \log _{10}\left(\tau \mu_{\mathrm{T}} \rho_{\mathrm{L}} P_{\mathrm{Tx}} G_{\mathrm{T}}^{2}\left|h_{i}\right|^{4}|\Gamma|^{2}\right) \\
& +40 \log _{10}\left(G_{\mathrm{R}}^{i}\right)+40 \log _{10}\left(L\left(d_{i}\right)\right)
\end{aligned}
$$

for $i=1, \cdots, N$. Note that, for monostatic configuration, $P_{i j}=0$ when $i \neq j$, for all $i, j \in\{1, \ldots, N\}$. Therefore, monostatic configuration essentially uses a subset of the antenna reader pairs in bistatic configuration during localization. In the rest of this paper, bistatic configuration as defined in 2.2 will be assumed to capture measurements at all pairwise combinations of antenna readers, including those corresponding to monostatic configurations.

\subsection{Tag / Reader Antenna Sensitivity and Localization Cov- erage}

In this section, we will first introduce the concepts of tag antenna sensitivity, reader antenna sensitivity, and localization coverage of an RFID system, which corresponds to the spatial region in which an RFID tag will be considered localizable.

\subsubsection{Tag Antenna Sensitivity}

The passive tags do not have an internal power structure to modulate or transmit any signal. They use the received power to both modulate the signal with the internal chip, and backscatter modulated signal to reader. As one can expect, RFID tags have certain power requirements. State-of-art tags are able to modulate signals with RSS as low as $-20 \mathrm{dBm}[51]$, and will not be able to detect the received signal at lower power levels. The RSS from $i$ th reader at tag is defined with

$$
P_{i}\left(x_{0}, y_{0}, z_{0}\right)=20 \log _{10}\left(\rho_{\mathrm{L}} P_{\mathrm{Tx}} G_{\mathrm{T}} G_{\mathrm{R}}^{i} L\left(d_{i}\right)\left|h_{i}\right|^{2}\right)
$$




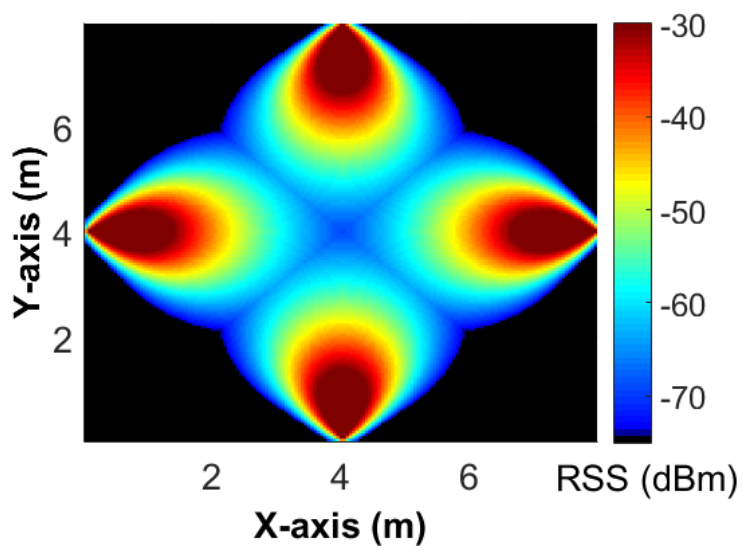

(a)

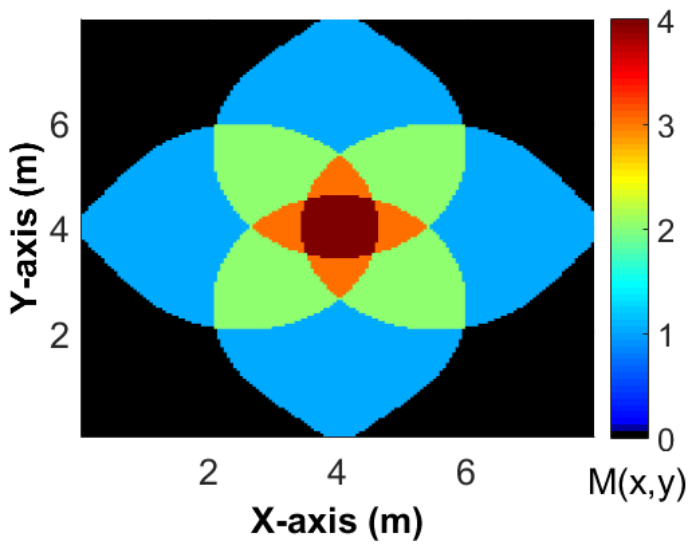

(b)

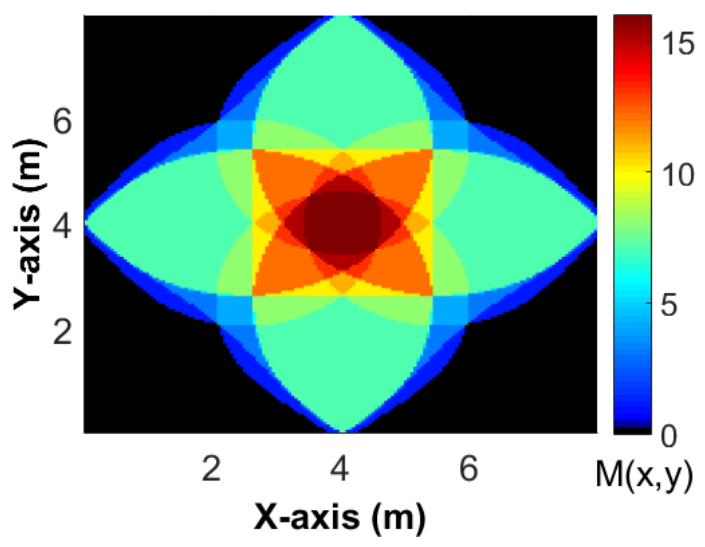

(c)

Figure 2.3: (a) Maximum achievable RSS at any possible tag location for the system. (b) Localization coverage for monostatic configuration. (c) Localization coverage for bistatic configuration. Areas where $M \geq 2$ are considered to be localizable, and the deployment parameters are $\theta_{i}=\pi / 4, H_{i}=1$ meter for $i=1,2,3,4$, and $P_{\mathrm{Tx}}=$ $1000 \mathrm{~mW}$. 
for $i=1, \cdots, N$. Note that $(2.10)$ is a subset of $(2.2)$ and $(2.9)$ which characterize the RSS after round-trip signal propagation, since 2.10 represents only the forward-link.

\subsubsection{Reader Antenna Sensitivity}

In either monostatic or bistatic configuration, an RFID reader must correctly detect the backscattered modulation from the tag, which relies on the reader antenna sensitivity. Therefore, the received power as in 2.2 and 2.9 must be larger than the reader antenna sensitivity in order to be detected. The detection coverage of an RFID configuration is defined as detectability of a tag at a certain location with that configuration. The detectability is assumed deterministic with respect to RSS and sensitivity of RFID reader antenna.

\subsubsection{Coverage Areas for Localization}

In this subsection, we investigate the impact of the sensitivity of the tag and reader antennas on localization performance. We introduce below several new metrics for characterizing tag/reader sensitivities and localization coverage.

Definition 1: The coverage for a given antenna pair $(i, j)$ at a given location $(x, y)$ is captured by a binary deterministic parameter $C_{i j}(x, y)$, which is defined as:

$$
C_{i j}(x, y)= \begin{cases}1, & \text { if } P_{i j} \geq-75 \mathrm{dBm} \text { and } P_{i} \geq-20 \mathrm{dBm} \\ 0, & \text { otherwise }\end{cases}
$$

using 2.2 and 2.10 .

Due to nonlinearity of antenna propagation model, in order to localize a tag, at least two different RSS measurements from that tag at a particular position $(x, y)$ are needed. On the other hand, there might be some tags which are detected from only a monostatic antenna or a bistatic antenna pair, and those tags cannot be localized 
due to limited information. Note that for monostatic configuration, $C_{i j}(x, y)=0$ if $i \neq j$. Then we can define the localizability of a tag as follows.

Definition 2: A tag at a given location $(x, y)$ is localizable if the following condition is satisfied

$$
M(x, y)=\sum_{i=1}^{N} \sum_{j=i}^{N} C_{i j}(x, y) \geq 2,
$$

where $N$ is the number of antennas in the system, and $M(x, y)$ is the total number antenna configurations that can detect the tag at location $(x, y)$.

Definition 3: The localization coverage of a tag at position $(x, y)$ is defined with $L(x, y)$ as follows:

$$
L(x, y)=\left\{\begin{array}{ll}
1, & \text { if } M(x, y) \geq 2 \\
0, & \text { otherwise }
\end{array} .\right.
$$

The RFID tag can be localized at position $(x, y)$ when $L(x, y)=1$, and is not localizable when $L(x, y)=0$.

Definition 4: The localization coverage percentage at a physical area $\mathcal{A}$ can be formally expressed as follows:

$$
L_{p}(\mathcal{A})=\frac{\iint_{x, \mathcal{A}} L(x, y) \mathrm{d} x \mathrm{~d} y}{\iint_{x, y \in \mathcal{A}} \mathrm{d} x \mathrm{~d} y} \times 100 \% .
$$

Note that 2.14 defines the percentage of localizable area to total area.

In Fig. 2.3, results from an example deployed scenario for parameters $\theta_{i}=\pi / 4$, $H_{i}=1$ meter for $i=1,2,3,4$, and $P_{\mathrm{Tx}}=1000 \mathrm{~mW}$ are shown. Maximum achievable RSS at any possible tag location is represented in Fig. 2.3(a), while monostatic and bistatic localization coverage are shown in Fig. 2.3(b) and Fig. 2.3(c), respectively. The localization coverage percentage is $21 \%$ for monostatic configuration, while it is above $50 \%$ for bistatic configuration. The number of maximum measurements increases from 4 for monostatic configuration to 16 for bistatic configuration. 


\subsection{Cramer-Rao Lower Bound and MLE}

The CRLB is a bound on the variance of any unbiased estimator for an unknown variable, such as the location of an RFID tag, based on a set of observations. In this section, we define likelihood function, and derive the CRLB on the accuracy of RSSbased UHF RFID localization systems as a function of various parameters of interest. We consider both monostatic and bistatic cases for CRLB analysis. Subsequently, the MLE for unknown RFID tag location is also defined. Comparison of CRLB and MLE for monostatic and bistatic configurations in various scenarios will be presented through numerical results in Section 2.7.

\subsubsection{Likelihood Function for Unknown RFID Tag Localiza- tion}

When a tag is localizable, then its exact location can be estimated using the measurements obtained at different antenna pairs. The probability of an RFID tag being at a certain location can be characterized by its likelihood function [52]. Let $\mathbf{x}=[x, y]$ denote the unknown location of the tag, assuming that the received power in log scale at an RFID reader antenna is subject to Gaussian noise [23]. Consider that the observations of received power in (2.2) from different RFID antennas mounted on the walls are stacked in a vector $\hat{\mathbf{p}}[\mathrm{dBm}]$. Then, this vector can be modeled as follows

$$
\hat{\mathbf{p}}=\mathbf{p}+\boldsymbol{\omega}, \boldsymbol{\omega}=\left[\omega_{11}, \ldots, \omega_{i j}, \ldots, \omega_{N N}\right]^{T}, \omega_{i j} \sim \mathcal{N}\left(0, \sigma^{2}\right),
$$

where $i=1, \ldots, N, j=1, \ldots, N$, and $\mathbf{p}$ is a vector of true RSS values which has a size of $N^{2}$ for a bistatic configuration, and a size of $N$ for a monostatic configuration. The additive noise on received power, which is assumed independent and identically distributed (iid), is captured by $\omega_{i j}$, corresponding to the measurement at 
antenna couple $i$ and $j$, with $\mathcal{N}\left(\mu, \sigma^{2}\right)$ denoting the Gaussian distribution with mean $\mu$ and variance $\sigma^{2}$. Then, for the general case of bistatic antenna configuration, the respective likelihood function for the received power at a location $\mathbf{x}$ can be written as:

$$
\begin{aligned}
\mathcal{L}(\hat{\mathbf{p}} ; \mathbf{x}) & =\frac{1}{\left(2 \pi \sigma^{2}\right)^{\frac{N^{2}}{2}}} \\
& \times \exp \left(-\frac{1}{2 \sigma^{2}} \sum_{i=1}^{N} \sum_{j=1}^{N} C_{i j}(x, y)\left(P_{i j}-\hat{P}_{i j}\right)^{2}\right),
\end{aligned}
$$

where $P_{i j}$ is the value of RSS for reader antennas $i$ and $j$, and it depends on the unknown tag location $\mathbf{x}=(x, y)$ as defined in 2.2 . For the monostatic configuration, it can be easily shown that 2.16 simplifies to the following likelihood function:

$$
\mathcal{L}(\hat{\mathbf{p}} ; \mathbf{x})=\frac{1}{\left(2 \pi \sigma^{2}\right)^{\frac{N}{2}}} \exp \left(-\frac{1}{2 \sigma^{2}} \sum_{i=1}^{N} C_{i i}(x, y)\left(P_{i i}-\hat{P}_{i i}\right)^{2}\right) .
$$

\subsubsection{CRLB Analysis}

Based on the 3D and directional propagation model defined in $2.1-(2.6)$, the localization coverage parameter $C_{i j}(x, y)$ defined in 2.11 , and the likelihood function defined in (2.16) the CRLB on the variance of an unbiased estimator for $\mathbf{x}$ can be defined as follows.

Theorem 1. The CRLB on the root mean square error (RMSE) of an unbiased position estimator $\hat{\mathbf{x}}$ based on the measurements model in 2.15 and the likelihood function in 2.16 is given by:

$$
\operatorname{RMSE}_{\mathrm{loc}}(x, y) \geq \sqrt{\mathbf{I}_{11}^{-1}+\mathbf{I}_{22}^{-1}}
$$

where $[\mathbf{I}(\mathbf{x})]$ is the Fisher Information Matrix (FIM) for $\mathbf{x}$,

$$
[\mathbf{I}(\mathbf{x})]=\left[\begin{array}{ll}
\mathbf{I}_{11} & \mathbf{I}_{12} \\
\mathbf{I}_{21} & \mathbf{I}_{22}
\end{array}\right]
$$


whose elements are as derived in (2.20)-(2.26).

\subsection{Proof of Theorem 1}

In this appendix we will show derivation of CRLB through obtaining FIM. Individual elements of the FIM in (2.19) can be calculated using the likelihood function $\mathcal{L}(\hat{\mathbf{p}} ; \mathbf{x})$ in (2.16) as follows [52]:

$$
\mathbf{I}_{m n}=-E\left[\frac{\partial^{2} \ln \mathcal{L}(\hat{\mathbf{p}} ; \mathbf{x})}{\partial \mathbf{x}_{m} \partial \mathbf{x}_{n}}\right],
$$

where $\mathbf{I}_{m n}$ is the $m n$-th element of the FIM for $m, n=1,2$. As in [52], using (2.16) the FIM element in 2.20 can be derived as follows. First, the natural logarithm of the likelihood function can be calculated as:

$$
\begin{aligned}
\ln \mathcal{L}(\hat{\mathbf{p}} ; \mathbf{x})= & \ln \left(\frac{1}{\left(2 \pi \sigma^{2}\right)^{\frac{N}{2}}}\right) \\
& -\frac{1}{2 \sigma^{2}} \sum_{i=1}^{N} \sum_{j=1}^{N} C_{i j}(x, y)\left(P_{i j}-\hat{P}_{i j}\right)^{2} .
\end{aligned}
$$

The partial derivatives of 2.21 should be taken with respect to each unknown pa-

rameter to get individual elements of the FIM. The derivative of (2.21) with respect to the first unknown parameter $\mathrm{x}_{m}$ can be written as

$$
\frac{\partial \ln \mathcal{L}(\hat{\mathbf{p}} ; \mathbf{x})}{\partial \mathbf{x}_{m}}=\frac{1}{\sigma^{2}} \sum_{i=1}^{N} \sum_{j=1}^{N} C_{i j}(x, y)\left(P_{i j}-\hat{P}_{i j}\right) \frac{\partial \hat{P}_{i j}}{\partial \mathbf{x}_{m}} .
$$

Then, the partial derivative of 2.22 with respect to $\mathrm{x}_{n}$ be calculated as

$$
\begin{aligned}
\frac{\partial^{2} \ln \mathcal{L}(\hat{\mathbf{p}} ; \mathbf{x})}{\partial \mathrm{x}_{m} \partial \mathrm{x}_{n}} & =\frac{1}{\sigma^{2}} \sum_{i=1}^{N} \sum_{j=1}^{N} C_{i j}(x, y) \\
\times & \left(-\frac{\partial \hat{P}_{i j}}{\partial \mathrm{x}_{n}} \frac{\partial \hat{P}_{i j}}{\partial \mathrm{x}_{m}}+\left(P_{i j}-\hat{P}_{i j}\right) \frac{\partial^{2} \hat{P}_{i j}}{\partial \mathrm{x}_{m}{ }^{2}}\right) .
\end{aligned}
$$

Getting the expectation of (2.23) with respect to $\mathrm{x}$ results

$$
E\left[\frac{\partial^{2} \ln \mathcal{L}(\hat{\mathbf{p}} ; \mathbf{x})}{\partial \mathbf{x}_{m} \partial \mathrm{x}_{n}}\right]=\frac{-1}{\sigma^{2}} \sum_{i=1}^{N} \sum_{j=1}^{N} C_{i j}(x, y)\left(\frac{\partial \hat{P}_{i j}}{\partial \mathbf{x}_{m}} \frac{\partial \hat{P}_{i j}}{\partial \mathbf{x}_{n}}\right) .
$$


Thus, the CRLB is a function of the first derivative of $(2.2)$ with respect to the unknown position parameter in logarithmic scale. Derivative of each element in received power is calculated separately since it can be written as summation of different functions in logarithmic scale. Partial derivative of 2.2 can be represented as

$$
\begin{aligned}
\frac{\partial \hat{P}_{i j}}{\partial \mathrm{x}_{m}} & =\frac{\partial\left(20 \log _{10}\left(\tau \mu_{\mathrm{T}} \rho_{\mathrm{L}} P_{\mathrm{Tx}} G_{\mathrm{T}}^{2}\left|h_{i} h_{j} \Gamma\right|^{2}\right)\right)}{\partial \mathrm{x}_{m}} \\
& +\frac{\partial\left(20 \log _{10} G_{\mathrm{R}}^{i}\right)}{\partial \mathrm{x}_{m}}+\frac{\partial\left(20 \log _{10} G_{\mathrm{R}}^{j}\right)}{\partial \mathrm{x}_{m}} \\
& +\frac{\partial\left(20 \log _{10} L\left(d_{i}\right)\right)}{\partial \mathrm{x}_{m}}+\frac{\partial\left(20 \log _{10} L\left(d_{j}\right)\right)}{\partial \mathrm{x}_{m}}
\end{aligned}
$$

The (unknown) location of the tag ( $\mathbf{x})$ does not affect the parameters $\tau \mu_{\mathrm{T}} \rho_{\mathrm{L}} P_{\mathrm{Tx}} G_{\mathrm{T}}^{2}\left|h_{i} h_{j} \Gamma\right|^{2}$ of received power, and hence the resulting partial derivative of $(2.2)$ is then given by

$$
\frac{\partial \hat{P}_{i j}}{\partial \mathrm{x}_{m}}=\frac{20}{\ln 10}\left(\frac{\partial G_{\mathrm{R}}^{i}}{\partial \mathrm{x}_{m}}+\frac{\partial G_{\mathrm{R}}^{j}}{\partial \mathrm{x}_{m}}+\frac{\partial L\left(d_{i}\right)}{\partial \mathrm{x}_{m}}+\frac{\partial L\left(d_{j}\right)}{\partial \mathrm{x}_{m}}\right) .
$$

\subsection{Example Derivation for CRLB}

In this appendix we will derive the CRLB for parameters $\theta_{i}=\pi / 4$ and $\phi_{i}=\pi / 2$ for $i=1,2,3,4$. The gain function in (2.6) for those particular values of $\theta_{i}$ and $\phi_{i}$ becomes

$$
G_{R}^{i}=3.136 \sin ^{2}\left(\frac{\sqrt{2} \pi\left(l_{i}+z_{i}-z_{0}\right)}{4 d_{i}}\right) \cos ^{2}\left(\frac{\sqrt{2} \pi\left(l_{i}-z_{i}+z_{0}\right)}{4 d_{i}} \frac{\left(y_{i}-y_{0}\right)}{l_{i}}\right) .
$$

First derivative of (2.27) with respect to $\mathrm{x}_{m}$, for $m=1,2$, is

$$
\frac{\partial G_{R}^{i}}{\partial \mathrm{x}_{m}}=\frac{3.136}{4} \times \frac{\partial B}{\partial \mathrm{x}_{m}} \sin A \sin (2 B) \times \frac{\partial A}{\partial \mathrm{x}_{m}} \sin (2 A) \cos B
$$

where

$$
\begin{aligned}
A & =\frac{\sqrt{2} \pi\left(l_{i}+z_{i}-z_{0}\right)}{4 d_{i}}, \\
B & =\frac{\sqrt{2} \pi\left(l_{i}-z_{i}+z_{0}\right)}{4 d_{i}} \frac{\left(y_{i}-y_{0}\right)}{l_{i}} .
\end{aligned}
$$


Then for $\mathrm{x}_{1}=x$ in $2.28, \frac{\partial A}{\partial x}$ and $\frac{\partial B}{\partial x}$ can be solved as

$$
\begin{aligned}
\frac{\partial A}{\partial x} & =\frac{\pi}{2 \sqrt{2}}\left(\frac{x-x_{i}}{l_{i} d_{i}}-\frac{\left(x-x_{i}\right)\left(l_{i}+z-z_{i}\right)}{d_{i}^{3}}\right) \\
\frac{\partial B}{\partial x} & =\frac{\pi\left(x-x_{i}\right)\left(y-y_{i}\right)}{2 \sqrt{2} l_{i} d_{i}}\left(\frac{1}{l_{i}}-\frac{\left(l_{i}-z+z_{i}\right)\left(l_{i}^{2}+d_{i}^{2}\right)}{l_{i}^{2} d_{i}^{2}}\right) .
\end{aligned}
$$

The same solution for $\mathrm{x}_{2}=y$ is given in

$$
\begin{aligned}
\frac{\partial A}{\partial y} & =\frac{\pi}{2 \sqrt{2}}\left(\frac{y-y_{i}}{l_{i} d_{i}}-\frac{\left(y-y_{i}\right)\left(l_{i}+z-z_{i}\right)}{d_{i}^{3}}\right), \\
\frac{\partial B}{\partial y} & =\frac{\pi\left(y-y_{i}\right)\left(l_{i}-z+z_{i}\right)}{2 \sqrt{2} l_{i} d_{i}} \\
& \times\left(\frac{1}{l_{i}\left(l_{i}-z+z_{i}\right)}-\frac{1}{l_{i}^{2}}-\frac{1}{d_{i}^{2}}-\frac{1}{\left(y-y_{i}\right)^{2}}\right) .
\end{aligned}
$$

The path loss function $L\left(d_{i}\right)$ does not change with $\theta$ and $\phi$, and it only depends on the distance between the reader antenna and the tag. Then, the derivative of $L\left(d_{i}\right)$ with respect to $x$ and $y$ is as follows

$$
\begin{aligned}
& \frac{\partial L\left(d_{i}\right)}{\partial x}=\frac{\lambda^{2}}{(4 \pi)^{2}}\left(\frac{x-x_{i}}{d_{i}^{3}}\right) \\
& \frac{\partial L\left(d_{i}\right)}{\partial y}=\frac{\lambda^{2}}{(4 \pi)^{2}}\left(\frac{y-y_{i}}{d_{i}^{3}}\right) .
\end{aligned}
$$

Based on these derivations, using (2.18)-(2.26), the CRLB for any location can be calculated with known set $x_{i}$ and $y_{i}$ for $i=1, \ldots, N$ with given parameters $\theta_{i}=\pi / 4$ and $\phi_{i}=\pi / 2$. In Fig. 2.5(c) and Fig. 2.5(d), CRLB for monostatic and bistatic configurations respectively are calculated for any possible location of tag.

\subsubsection{Maximum Likelihood Estimator}

While the CRLB gives a lower bound on the localization RMSE, an effective estimator is needed to find an RFID tag's location as accurate as possible, ideally with an RMSE close to the CRLB. In here, we will define a simple MLE estimator for comparison 
purposes with the CRLB. Using the likelihood function defined in (2.16), the MLE can be formulated as follows [52]

$$
\hat{\mathbf{x}}=\arg \max _{\mathbf{X}} \mathcal{L}(\mathbf{P} ; \hat{\mathbf{x}}) .
$$

Having a closed form solution for the MLE in (2.29) is not mathematically tractable due to the complexity of the directional antenna radiation pattern as captured through (2.2)-2.6). In particular, due to entangled sines and cosines, after equating differentiation of the likelihood function as in (2.26) to zero, one cannot obtain a closed form solution. Thus our problem could be solved with MLE grid search, which can be represented as follows

$$
\hat{\mathbf{x}}=\arg \min _{\mathbf{X}} \sum_{i=1}^{N} \sum_{j=1}^{N}\left(P_{i j}(x, y)-\hat{P}_{i j}(x, y)\right)^{2} .
$$

In our computer simulations in Section 2.7, we consider a densely sampled grid of nearly 15000 uniformly spaced points. The granularity of the grid is set to 5 $\mathrm{cm}$. Then, the MLE solution corresponds to the grid position that maximizes the likelihood function in (2.16) and can be found using exhaustive search. To reduce complexity, the MLE solution is found by a constrained search over the region that is defined by the number of RSS measurements and corresponding antennas. When there are only two RSS measurements available, the search is conducted only over the positions where $M(x, y)=2$. As it is stated in Section 2.3.3, a grid location with only two RSS measurements is still localizable, although the accuracy is relatively limited when compared to locations where more than two RSS measurements are available. Based on our numerical results that will be shown in Section 2.7, overall localization accuracy is still acceptable. Accuracy of the MLE will be compared with the CRLB in various scenarios in the next section. 
Table 2.2: Passive UHF RFID system parameters.

\begin{tabular}{|l|l|}
\hline Parameter & Value \\
\hline \hline Operating Frequency & $865.7 \mathrm{MHz}$ \\
\hline Operating Bandwidth & $300 \mathrm{kHz}$ \\
\hline Transmit Power $\left(P_{\mathrm{Tx}}\right)($ EIRP $)$ & $1000 \mathrm{~mW}$ to $3000 \mathrm{~mW}$ \\
\hline Modulation Efficiency $(\tau)$ & 0.5 \\
\hline Polarization Loss Factor $\left(\rho_{\mathrm{L}}\right)$ & 0.5 \\
\hline Power Transfer Efficiency $\left(\mu_{\mathrm{T}}\right)$ & 0 to 1 \\
\hline Differential Reflection Coefficient $\left(|\Gamma|^{2}\right)$ & 0 to 1 \\
\hline Tag Antenna Gain $\left(G_{\mathrm{T}}\right)$ & $0 \mathrm{dBi}$ \\
\hline Tag Antenna Sensitivity $\left(G_{\mathrm{T}}\right)$ & $-18 \mathrm{dBm}$ \\
\hline Reader Antenna Sensitivity $\left(R_{\mathrm{S}}\right)$ & $-75 \mathrm{dBm}$ \\
\hline Antenna Height & $2 \mathrm{~m}$ \\
\hline Tag Height & $1 \mathrm{~m}$ \\
\hline Room Width and Length & $8 \mathrm{~m} \mathrm{x} 8 \mathrm{~m}$ \\
\hline Granularity of Simulations & $1 \mathrm{~cm}$ \\
\hline Reader Antenna Elevation Angle $(\theta)$ & $\pi / 4$ to $\pi / 2$ \\
\hline
\end{tabular}

\subsection{Numerical Results}

Numerical results are provided to validate analytic derivations with computer simulations and to compare the performance of the MLE with the CRLB for RFID based IoT localization. The simulation parameters for the passive UHF RFID system is given in Table 2.2, As stated in Section 2.4, the received power at the RFID reader antenna is subject to lognormal noise. The noise variance is adopted from the statistical models in [43,53], which were derived from RFID propagation measurements.

Our computer simulation considers RFID antennas that are installed in a square shaped room with 8 meters width, and the height of the reader antennas are 2 meters above floor level. The channel is assumed to be frequency flat slow fading channel in our system. There are three antenna placement configurations, first is placing the antennas to centers of side walls which is referred as 'Side', the second is placing them on the corners of the room which is referred as 'Corner' in figures, and the 

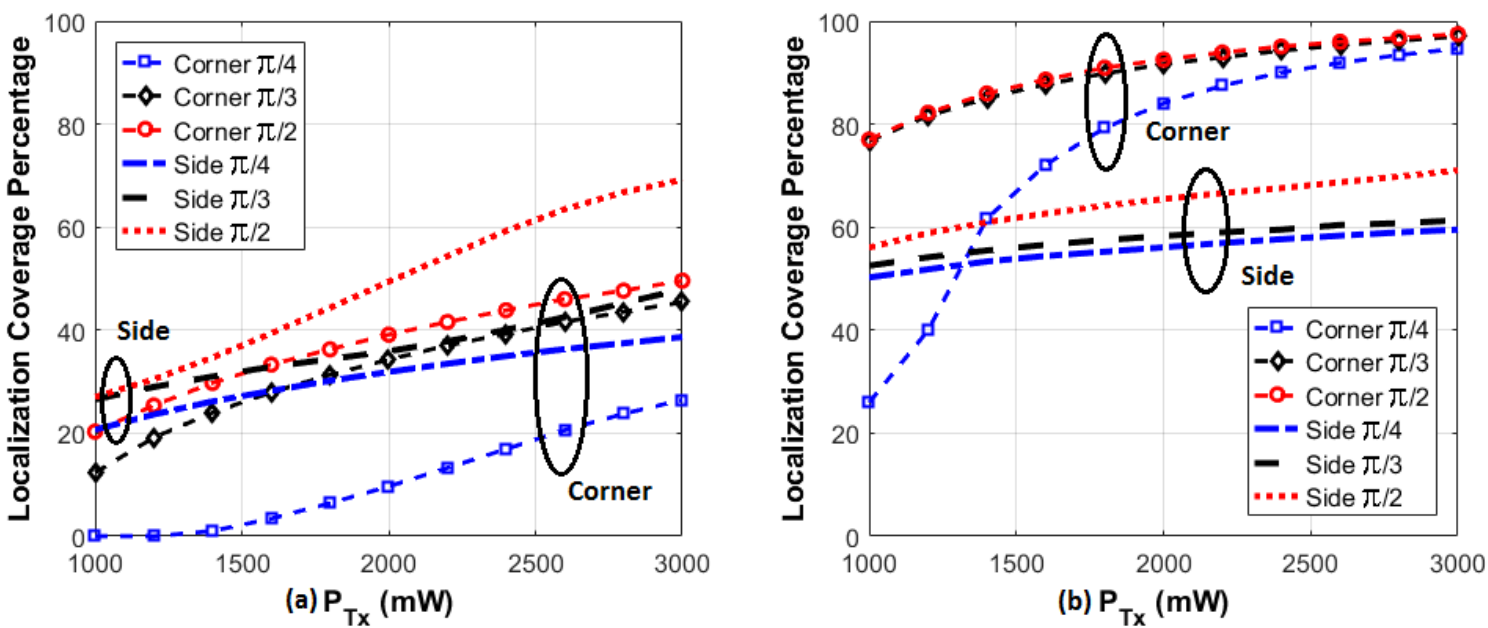

Figure 2.4: Localization coverage percentage for $\theta_{i}=\pi / 4, \pi / 3, \pi / 2$, and $i=1,2,3,4$, (a) monostatic and (b) bistatic configurations.

third is placing them on the line of a side wall as an equally spaced array which is referred as 'Array' in results. The reader uses circularly polarized antennas which have a radiation pattern as defined in (2.5), and the tag antennas are assumed to be vertically polarized. The height of the tag is assumed to be known and 1 meter. Elevation angles of reader antennas are defined as $\pi / 4, \pi / 3$, and $\pi / 2$. Elevation angles lower than $\pi / 4$ are not considered due to lack of localization coverage for those angles.

\subsubsection{Localization Coverage}

In Fig. 2.4, localization coverage percentage in (2.14) is illustrated for different elevation angles, antenna placement configurations, and transmit power levels for monostatic (Fig. 2.4(a)) and bistatic (Fig. 2.4(b)) antenna configuration. The localization coverage is below $50 \%$ for monostatic cases other than Side $\pi / 2$. The coverage percentage for monostatic configuration increases rapidly with increasing transmit power from $17.8 \%$ on the average for $P_{\mathrm{Tx}}=1000 \mathrm{~mW}$ to $46.2 \%$ for $P_{\mathrm{Tx}}=3000 \mathrm{~mW}$ 
transmit power. Localization coverage for bistatic cases show improvement with increased transmit power as well. The mean localization coverage percentage for $P_{\mathrm{Tx}}=1000 \mathrm{~mW}$ is $56.4 \%$, while increasing transmit power to $P_{\mathrm{Tx}}=3000 \mathrm{~mW}$ substantially boosts it to $80.2 \%$.

The elevation angle also plays a critical role in localization coverage of the system. In monostatic and bistatic configurations, $\theta=\pi / 2$ is superior to other angles for both Corner and Side placement of antennas. In general, the coverage is increased with increased elevation angle. Corner placement of the antennas is better in bistatic configuration, whereas in monostatic configuration side placement has larger coverage area in general. The corner placement of the antennas covers $85 \%$ of the area for bistatic configuration on the average for all available transmit powers, whereas side placement enables to localize the tags in $59.4 \%$ of the area. Things are different for monostatic case, where corner placement has $27 \%$ coverage, while side placement achieves better performance with $38.9 \%$. This is expected since side placement increases the overlap possibility of monostatic antenna coverages with less distances between antennas, whereas corner placement exploits the radiation coverage with increased distances between antennas.

\subsubsection{Localization Accuracy}

In Fig. 2.5, Average MLE and CRLB RMSE for monostatic and bistatic configurations with $\theta=\pi / 4$, for $P_{\mathrm{Tx}}=1000 \mathrm{~mW}$ at each possible tag location is given. The localization coverage for monostatic configuration is just above $20 \%$, while in bistatic configuration it is above $50 \%$ as represented in Fig. 2.4. Monostatic configuration has localization coverage above $50 \%$ for only side placement of antennas with $\theta=\pi / 2$, thus they are not represented in median localization RMSE results which they do not have. 


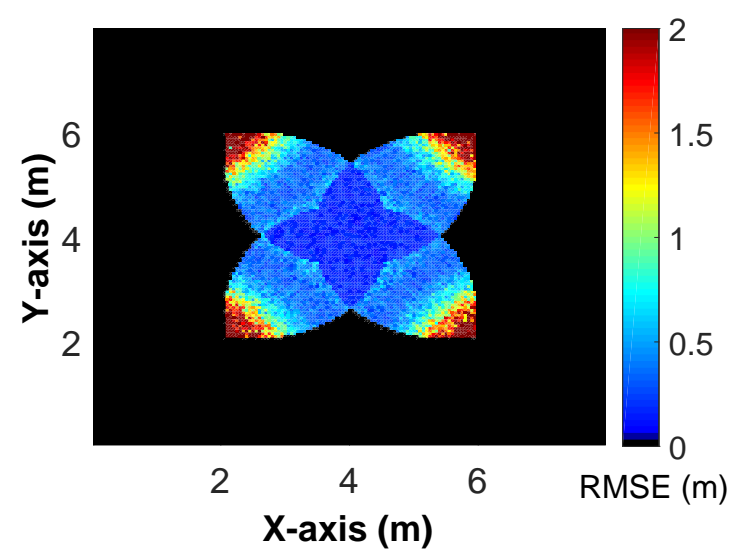

(a) MLE Monostatic

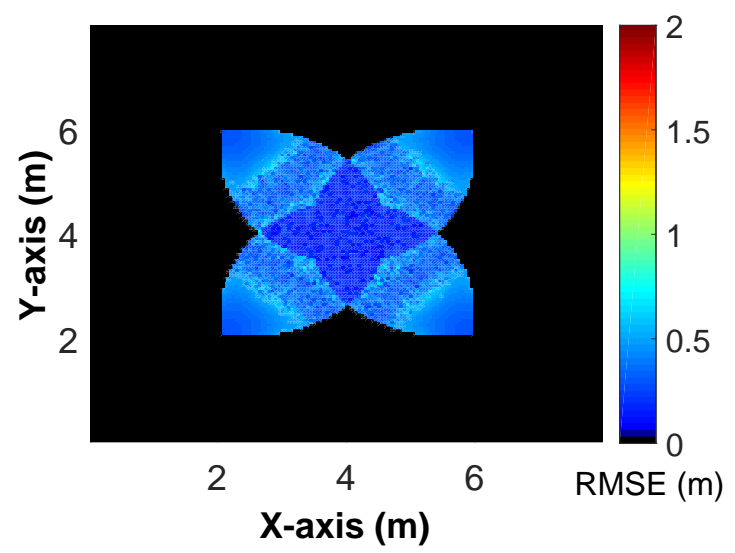

(c) CRLB Monostatic

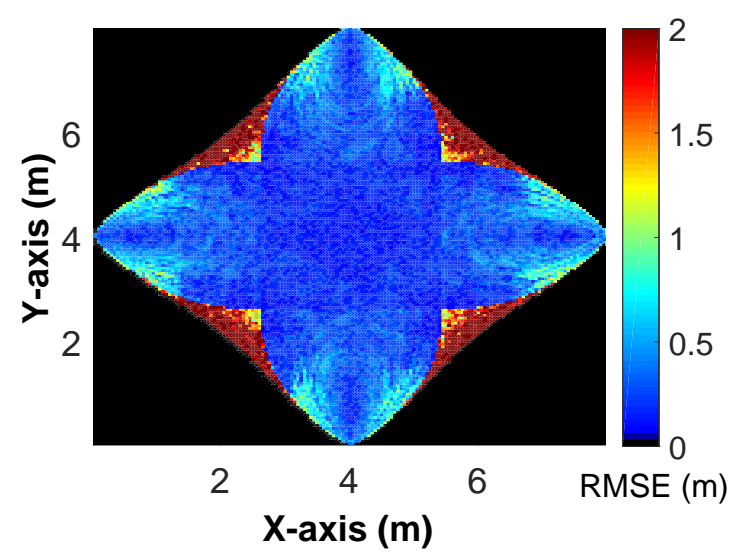

(b) MLE Bistatic

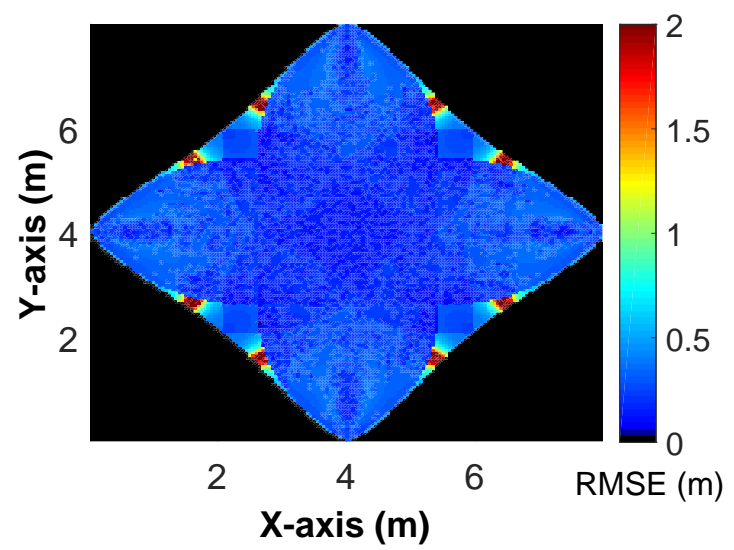

(d) CRLB Bistatic

Figure 2.5: Average MLE and CRLB RMSE for monostatic and bistatic configurations for $\theta_{i}=\pi / 4, H_{i}=1$ meter, for $i=1, \ldots, 4$.

The median localization RMSE of CRLB and MLE are compared in Fig. 2.6(a), for elevation angle of $\theta=\pi / 4$. Monostatic configuration is not in the results since it does not have a coverage above $50 \%$ as in Fig. 2.4. Median RMSE of CRLB for side placement of antennas begin with 1.07 meters at $P_{\mathrm{Tx}}=1200 \mathrm{~mW}$ and gets as low as 0.72 meters, while corner placement has lower median error in general from 0.61 meters at $P_{\mathrm{Tx}}=1400 \mathrm{~mW}$ to 0.43 meters at $P_{\mathrm{Tx}}=3000 \mathrm{~mW}$. As expected, MLE gets closer performance to the CRLB as transmit power increases. Median RMSE of 

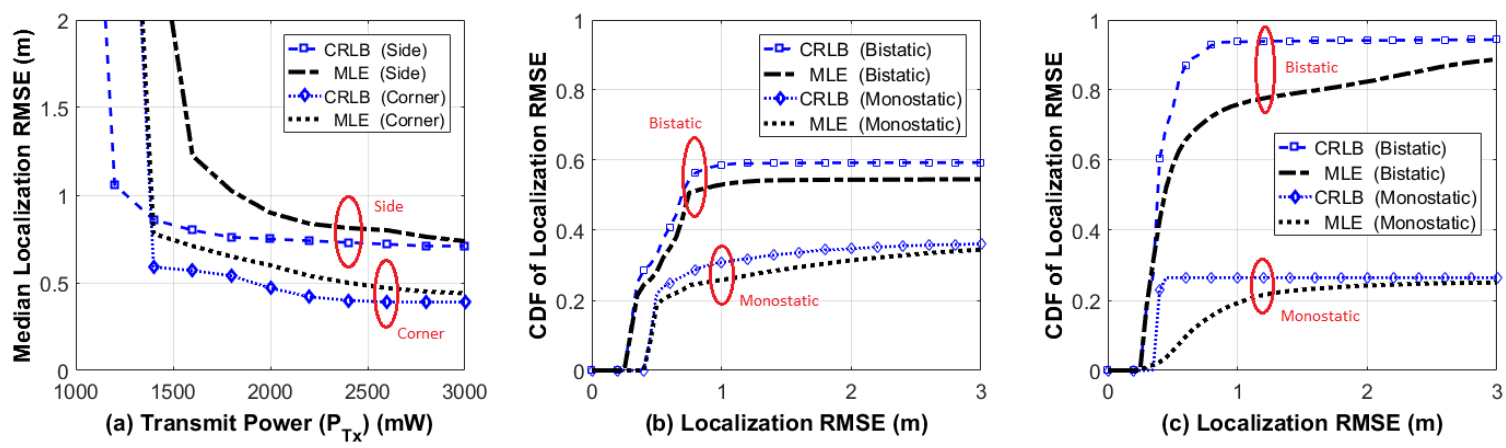

Figure 2.6: Deployment with $\boldsymbol{\theta}_{\boldsymbol{i}}=\boldsymbol{\pi} / \boldsymbol{4}$ for $i=1,2,3,4$, (a) Median localization RMSE of MLE and CRLB for various transmit powers, (b) CDF of RMSE of MLE and CRLB with side placement, (c) CDF of RMSE of MLE and CRLB with corner placement.

MLE for side placement of antennas begin with 1.26 meters at $P_{\mathrm{Tx}}=1600 \mathrm{~mW}$ to 0.76 meters at $P_{\mathrm{Tx}}=3000 \mathrm{~mW}$, while corner placement does better with 0.73 meters at $P_{\mathrm{Tx}}=1400 \mathrm{~mW}$, and 0.45 meters at $P_{\mathrm{Tx}}=3000 \mathrm{~mW}$.

In Fig. 2.6(b), performance of side placement, and in Fig. 2.6(c), performance of corner placement is are shown. In Fig. 2.6(b), the localization probability of a tag with MLE below an error of 1 meter for monostatic configuration with side placement and $P_{\mathrm{Tx}}=3000 \mathrm{~mW}$ is 0.26 , while for bistatic configuration with same parameters it gets to 0.53. The CDF values of CRLB for those are 0.31 and 0.59 , respectively.

In Fig. 2.6(c), the localization probability of a tag with MLE below an error of 1 meter for monostatic configuration with corner placement and $P_{\mathrm{Tx}}=3000 \mathrm{~mW}$ is 0.19 , while for bistatic configuration with same parameters it gets to 0.76. The CDF values of CRLB for those are 0.26 and 0.92 , respectively. The side placement of antennas has better performance with monostatic MLE compared to corner placement, while bistatic performance substantially lower.

Increasing elevation angle to $\theta=\pi / 3$ helps to decrease median localization RMSE and improve localization performance. The median localization RMSE of CRLB and MLE are compared in Fig. 2.7(a), for elevation angle of $\theta=\pi / 3$. As shown 

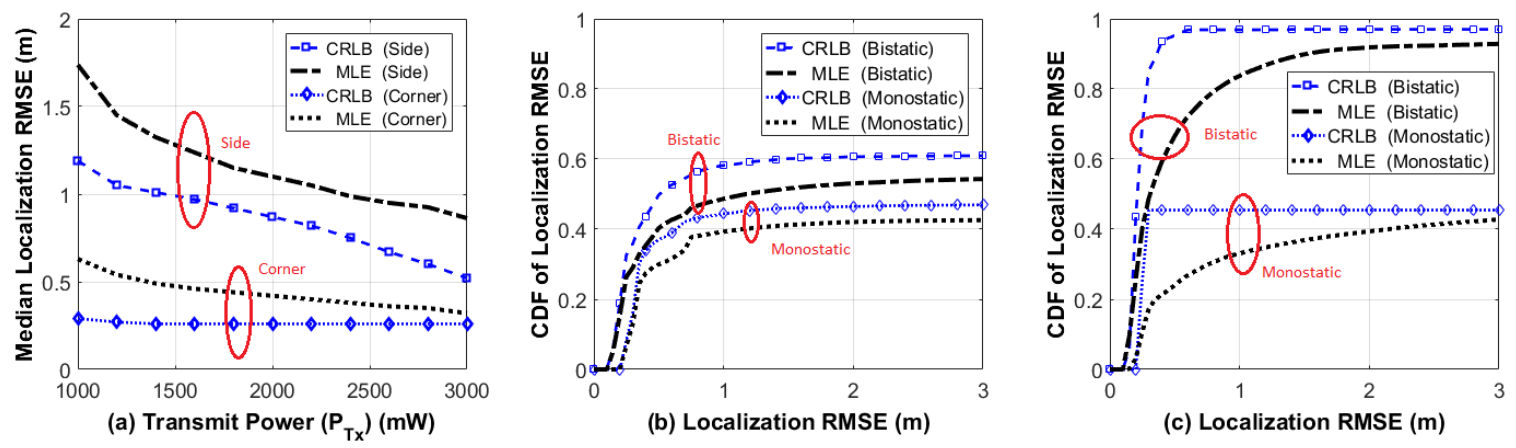

Figure 2.7: Deployment with $\boldsymbol{\theta}_{\boldsymbol{i}}=\boldsymbol{\pi} / \mathbf{3}$ for $i=1,2,3$, 4, (a) Median localization RMSE of MLE and CRLB for various transmit powers, (b) CDF of RMSE of MLE and CRLB with side placement, (c) CDF of RMSE of MLE and CRLB with corner placement.

in Fig. 2.4, bistatic configuration is always above $50 \%$ in localization coverage. In Fig. 2.7(a), median RMSE of CRLB for side placement of antennas begin with 1.21 meters at $P_{\mathrm{Tx}}=1000 \mathrm{~mW}$ and gets as low as 0.51 meters, while corner placement has lower median error in general from 0.32 meters to 0.3 meters at $3000 \mathrm{~mW}$. Similar to $\theta=\pi / 4$, MLE converges to CRLB as transmit power increases. Median RMSE of MLE for side placement of antennas begin with 1.71 meters at $P_{\mathrm{Tx}}=1000 \mathrm{~mW}$, which reduces to 0.78 meters at $P_{\mathrm{Tx}}=3000 \mathrm{~mW}$, while corner placement does better with 0.63 meters and 0.34 meters, respectively.

In Fig. 2.7(b) CDF of localization RMSE for side placement is shown for side placement with $\theta=\pi / 3$. The localization probabilities of a tag below an error of 1 meter for monostatic and bistatic configuration are 0.33 and 0.84 , respectively, while their CRLB are 0.44 and 0.97 , respectively.

In Fig. 2.7(c), the localization probability of a tag with MLE below an error of 1 meter for monostatic configuration with corner placement and $P_{\mathrm{Tx}}=3000 \mathrm{~mW}$ are 0.41 and 0.51 , while their CRLB are 0.44 and 0.58 , respectively. Side placement of antennas increase the performance of monostatic configuration while degrading bistatic configuration performance similar to $\theta=\pi / 4$. 

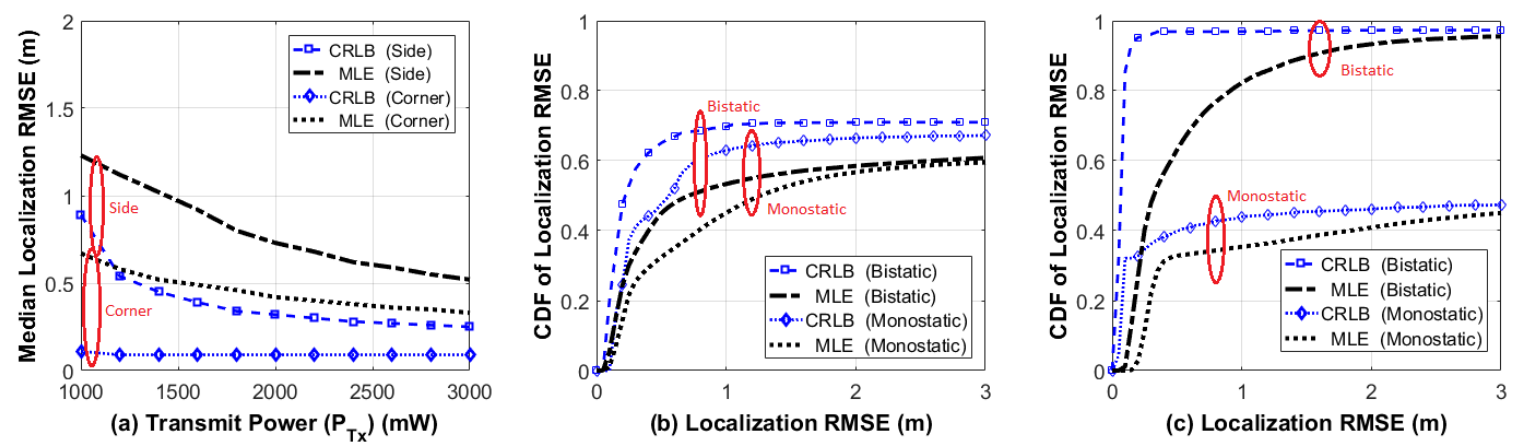

Figure 2.8: Deployment with $\boldsymbol{\theta}_{\boldsymbol{i}}=\boldsymbol{\pi} / \mathbf{2}$ for $i=1,2,3$, 4, (a) Median localization RMSE of MLE and CRLB for various transmit powers, (b) CDF of RMSE of MLE and CRLB with side placement $\left(P_{\mathrm{Tx}}=3000 \mathrm{~mW}\right)$, (c) CDF of RMSE of MLE and CRLB with corner placement and $\left(P_{\mathrm{Tx}}=3000 \mathrm{~mW}\right)$.

In Fig. 2.8(a), the median localization RMSE of CRLB and MLE are compared for elevation angle of $\theta=\pi / 2$ with side and corner placement of antennas. Median RMSE of CRLB for side placement of antennas begin with 0.89 meters at $P_{\mathrm{Tx}}=$ $1000 \mathrm{~mW}$ and gets as low as 0.25 meters, while corner placement has lower median error in general from 0.11 meters to 0.09 meters at $3000 \mathrm{~mW}$. Similar to $\theta=\pi / 4$ and $\theta=\pi / 3$, MLE converges to CRLB as transmit power increases. Median RMSE of MLE for side placement of antennas begin with 1.23 meters at $P_{\mathrm{Tx}}=1000 \mathrm{~mW}$ and reduce to 0.52 meters at $P_{\mathrm{Tx}}=3000 \mathrm{~mW}$, while corner placement does better with 0.67 meters and 0.33 meters, respectively.

In Fig. 2.8(b) CDF of localization RMSE for side placement is represented. The localization probabilities of a tag below an error of 1 meter for monostatic and bistatic configuration are 0.35 and 0.82 , respectively. The CRLB for those are 0.44 and 0.97 , respectively. In Fig. 2.8(c), the localization probability of a tag with MLE and CRLB is shown with respect to localization RMSE. The probability of having an error below 1 meter for monostatic configuration with corner placement and $P_{\mathrm{Tx}}=3000 \mathrm{~mW}$ are 0.47 and 0.56 , while the CDF of CRLB for those are 0.63 and 0.70 , respectively. Side 


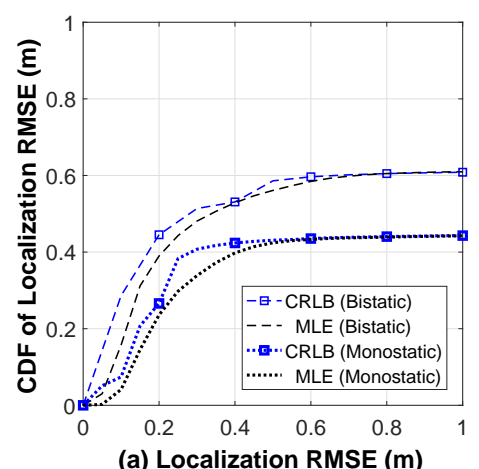

(a) Localization RMSE (m)

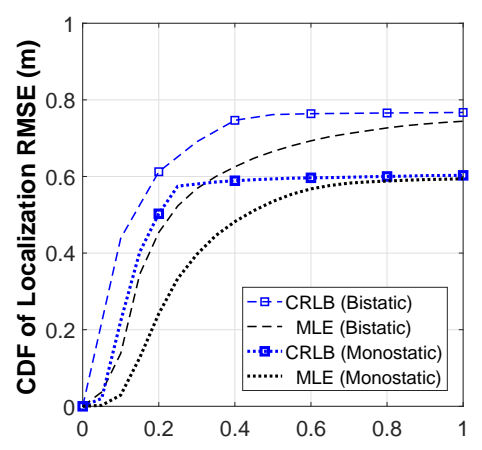

(b) Localization RMSE (m)

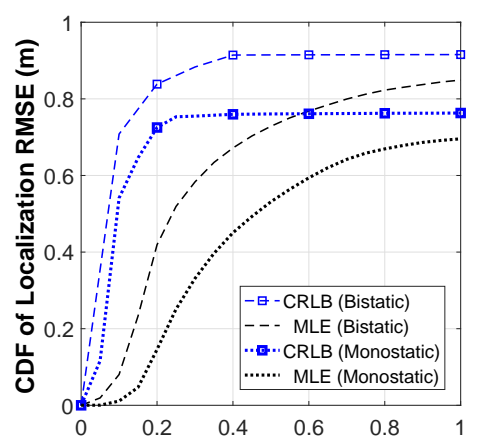

(c) Localization RMSE (m)

Figure 2.9: CDF of RMSE of MLE and CRLB with linear placement of antennas for deployment with (a) $\boldsymbol{\theta}_{\boldsymbol{i}}=\boldsymbol{\pi} \mathbf{4}$, (b) $\boldsymbol{\theta}_{\boldsymbol{i}}=\boldsymbol{\pi} / \mathbf{3}$, and (c) $\boldsymbol{\theta}_{\boldsymbol{i}}=\boldsymbol{\pi} / \mathbf{2}$ for $i=1,2,3,4$ $\left(P_{\mathrm{Tx}}=3000 \mathrm{~mW}\right)$.

placement of antennas increase the performance of monostatic configuration slightly while degrading bistatic configuration performance substantially.

In Fig. 2.9, a linear array of antennas is considered, where RFID reader antennas are placed equally spaced on a single side-wall of the room. The CDF of the localization RMSE for array placement with $\theta=\pi / 4$ is shown in Fig. 2.9(a). The localization probabilities of a tag below an error of 1 meter for monostatic and bistatic configuration are 0.46 and 0.60 , respectively. In Fig. 2.9(b), CDF of the localization RMSE for array placement with $\theta=\pi / 3$ is provided. The localization coverage is increased significantly with increasing elevation angle. The localization probabilities of a tag below an error of 1 meter for monostatic and bistatic configuration are 0.59 and 0.74 , respectively. The CRLB for those are 0.61 and 0.77 . Increasing the elevation angle to $\theta=\pi / 2$ gives the best results for localization coverage and localization accuracy as shown in Fig. 2.9(c). The localization probabilities of a tag below an error of 1 meter for monostatic and bistatic configuration are 0.70 and 0.85 , respectively, and the corresponding CRLB are 0.76 and 0.92, respectively. Array placement of antennas results in a performance in between side and corner placement, and provides a favorable alternative for lengthy and narrow areas. 
In general, configurations with larger elevation angle results better localization coverage and lower localization RMSE. In Fig. 2.6(a), the median localization RMSE for $\theta=\pi / 4$ has much higher values compared to $\theta=\pi / 3$ in Fig. 2.7(a) and $\theta=\pi / 2$ in Fig. 2.8(a), for example, at $P_{\mathrm{Tx}}=1000 \mathrm{~mW}$ localization RMSE is not available for $\theta=\pi / 4$ since its localization coverage is all below $50 \%$ for either corner and side placement of antennas, while $\theta=\pi / 3$ and $\theta=\pi / 2$ have acceptable accuracies. Especially $\theta=\pi / 2$ has median localization RMSE of 0.5 meters for both side and corner configuration. At all elevation angles, corner placement of antennas has better localization coverage for bistatic configuration at $P_{\mathrm{Tx}}=3000 \mathrm{~mW}$. Monostatic configuration does better with side placement of antennas, since in that case the coverage of antennas overlaps in larger areas. Increasing transmit power not only increases the localization coverage, but also reduces the localization error. As a conclusion, an elevation angle larger than $\theta=\pi / 3$ is crucial for localization coverage and accuracy as well as corner placement of antennas with transmit power at $3000 \mathrm{~mW}$ which is the EIRP limit in EPC Gen2 protocol of UHF RFID systems. 


\section{CHAPTER 3}

\section{RSS-BASED MMWAVE LOCALIZATION}

The rapid increase in the bandwidth hungry applications for smart devices results in a crisis because of the sparsely available spectrum [54]. Utilization of the millimeter wave (mmWave) band is the promising solution for these bandwidth hungry future of the communication systems. It makes use of larger bandwidths at frequencies above $30 \mathrm{GHz}$ for enabling higher data rates for various applications [55]. The mmWave will be one of the integral parts for $5 \mathrm{G}$ cellular networks because of the available spectrum at the higher frequency bands, and the possibility of spatial reuse due to penetration losses. Other than cellular considerations, it is already being used in indoor wireless backhaul technologies for high capacity low latency connectivity [56,57]. As an example, IEEE 802.11ad is a recent wireless mmWave-based standard, that can support wireless HDMI connectivity or personal docking stations to allow indoor mobility for smart devices.Beamforming is an essential component of directional communication at mmWave bands to overcome excessive path and penetration losses. The short wavelengths of the mmwave has facilitated antenna arrays embedded into portable devices with a compact form factor, making beamforming possible. IEEE 802.11ad has directional multi-gigabit access as a core feature, which is built on multiple-antenna beamforming with sector level sweep (SLS) in $60 \mathrm{GHz}$ [58]. SLS is not only a fast solution for beam-steering, but is also a good candidate for localization applications.

While the use of mmwave for cellular communications have recently received extensive interest, its use for localization is relatively unvisited. In [59], authors compare several mmwave localization approaches considering psuedo-omnidirectional antenna radiation pattern. In [60], the authors propose triangulation-validation, angledifference-of-arrival, and location fingerprinting. A high accuracy localization method for assisted living systems considered in [61] using a multipath-assisted environment- 
aware technique. The beamforming capabilities with large signal bandwidth of $5 \mathrm{G}$ are envisioned as a great enabler for robust and accurate indoor localization even with a single anchor node. An RSS-based mmWave indoor localization is proposed in 62 assuming omni-directional antennas at both ends and LOS conditions.

In this work, we study the fundamental limits of RSS-based mmWave positioning systems using beamforming considering indoor scenarios for IEEE 802.11ad SLS. To the best knowledge of the authors, there is no work that studies the fundamental limits of RSS-based mmwave localization explicitly considering beamforming radiation pattern. We derive the Cramer-Rao Lower Bound on the localization accuracy, and propose a nonlinear least squares estimator (NLSE). The main contributions of this work are as follows: 1) A realistic closed-form RSS equation is derived considering radiation pattern based on antenna array factor; 2) Fundamental limits of localization accuracy for RSS-based mmWave positioning system are derived in closed form with a realistic antenna array model; 3) A NLSE is proposed for localization of the user device and performance is compared to fundamental limits.

\subsection{System Model}

Consider a mmWave communication system where the base station (BS) has a linear antenna array of $N$ antennas and the user equipment (UE) has an omnidirectional antenna as in Fig. 3.1. The spacing between each antenna element at BS is uniform and denoted with $d_{s}$. We assume each antenna element can be excited individually to enable desired beams. The BS creates beams as in SLS at discrete angles based on number of antenna elements to gather RSS measurements from the UE. The BS is located at known position $\mathbf{x}_{\mathbf{b}}=\left[x_{b}, y_{b}\right]$ and UE is located at unknown position $\mathbf{x}=[x, y]$ in an $l \times l$ square area of the two-dimensional plane in a room. The distance between BS and UE is defined as $d=\left\|\mathbf{x}_{\mathbf{b}}-\mathbf{x}\right\|$. RSS varies by the distance 


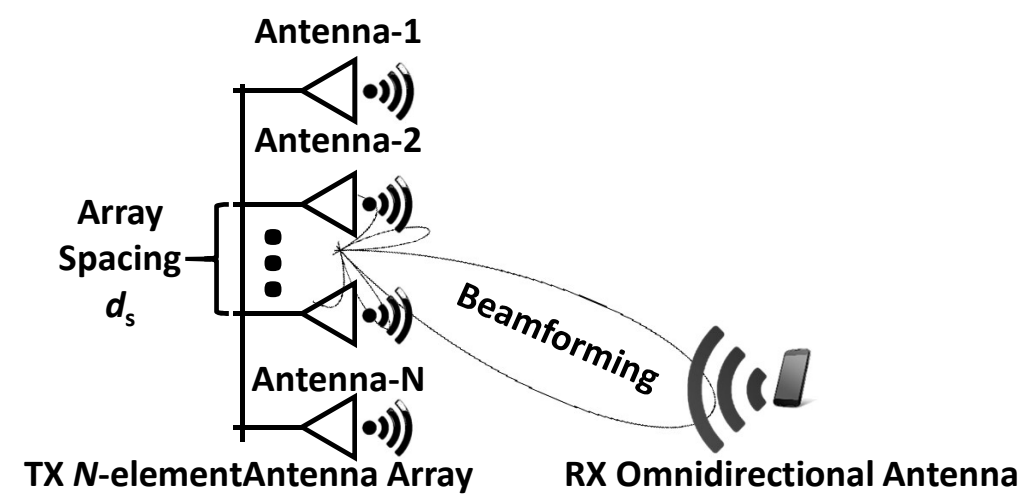

Figure 3.1: An omnidirectional transmitter antenna at UE and $N$-element receiver antenna array at BS as IEEE 802.11ad mmWave communication system.

between BS and UE, and the gain of the antenna array. RSS is explicitly defined by the common model of Friis' free space path loss law 55 considering $i$ th beam direction as follows

$$
\tilde{P}_{i}\left(d, \phi_{i}\right)=\frac{P_{t} G_{t}(\phi) G_{r}}{L}\left(\frac{\lambda}{4 \pi d}\right)^{2},
$$

where $P_{t}$ is the transmit power, $G_{t}(\phi)$ is the transmit gain, $G_{r}$ is the receiver antenna gain, $\lambda$ is the wavelength of the signal, and $L$ stands for losses of the mmWave system. Considering (3.1), RSS in logarithmic scale can be written as

$$
P_{i}\left(d, \phi_{i}\right)[\mathrm{dBm}]=20 \log _{10}\left(\frac{P_{t} G_{r} \lambda^{2}}{16 L \pi^{2}}\right)+20 \log _{10} G_{t}\left(\phi_{i}\right)-40 \log _{10} d
$$

Gain of an antenna is defined by the product of antenna efficiency $\varepsilon$ and directivity $D(\phi)$ as $G(\phi)=\varepsilon_{r} D(\phi)$. The angle $\phi$ is the azimuthal angle between the direction of the antenna array and the location of UE as shown in Fig. 3.2 and defined as

$$
\phi=\cos ^{-1}\left(\frac{x-x_{b}}{d}\right)
$$

On the other hand, the directivity of an antenna can be defined using the Poynting vector, which represents directional flux density and denoted by $\boldsymbol{S}(\theta, \phi)$ where $\theta$ and $\phi$ representing elevation and azimuthal angles, divided by the aggregate radiated power. 


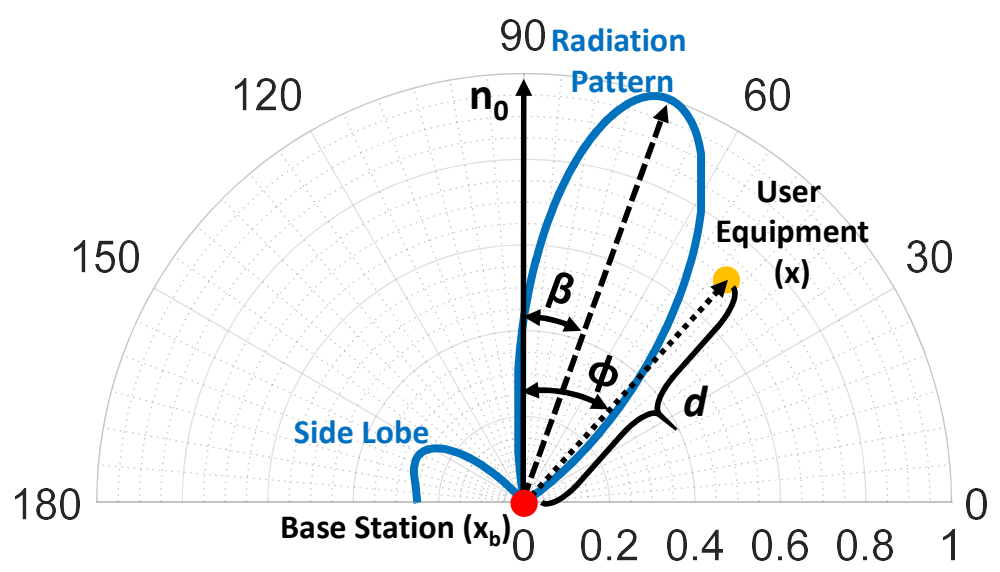

Figure 3.2: Azimuthal radiation pattern of linear antenna array and locations of BS and UE.

Directionality of the array is given by [63] $D_{\text {array }}(\phi)=A(\phi) \times D_{\text {eelm }}(\phi)$, where $A(\phi)$ is the array factor for the antenna which depends on arrangement of the antenna elements, and $D_{\text {elm }}(\phi)$ represents the directivity of each and every element of the antenna array. Considering $N$ antenna elements uniformly spaced on a linear array, the array factor can be defined as 63

$$
A(\phi)=\sum_{m=0}^{N-1} e^{j m \psi}=1+e^{j \psi}+e^{j 2 \psi}+\cdots+e^{j(N-1) \psi}
$$

where $\psi=k d_{s} \cos \phi+\beta, k=\frac{2 \pi}{\lambda}$, and $\beta$ is the desired phase difference for the beam as shown in Fig. 3.2. The magnitude of the array factor can be written in trigonometric equation as 63

$$
|A(\phi)|=\left|\frac{\sin \left(\frac{N \psi}{2}\right)}{\sin \left(\frac{\psi}{2}\right)}\right|=\left|\frac{\sin \left(\frac{N}{2} k d_{S} \cos \phi+\beta\right)}{\sin \left(\frac{1}{2} k d_{S} \cos \phi+\beta\right)}\right| .
$$

\subsection{Sector Level Sweeping}

In IEEE 802.11ad beamforming training, virtualized sectors are used to focus antenna gain in certain discrete directions. This process, referred to as beamforming training, takes advantage of the discretized antenna azimuth that reduces the search space of 


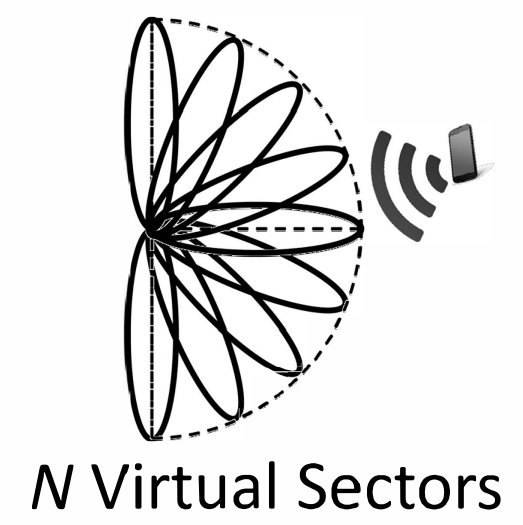

Figure 3.3: Sector Level Sweeping.

possible antenna array. After a first sector matching, a second beam training stage allows further refinement of the found sectors. During this stage, antenna weight vectors that vary from predefined sector patterns can be evaluated to further optimize transmissions on phased antenna arrays. In our work, we use the first stage of sector level sweeping to localize the user equipment by measuring RSS level at each sector. Sectors are defined by the number of antenna elements since using more antenna elements imposes higher directionality and narrower beams. In Fig. 3.3, there are 9 sectors presented at 9 discrete azimuth angles. Each of the beams are created using digital beamforming, and each of the RSS measurements are recorded for localization. If the UE is not in proximity of a sector, the RSS may not reach the threshold for detection. In such cases, we discard those sectors from measurements, and truncate the measurement vector accordingly.

\subsection{RSS-Based Localization}

Under the assumption of availability of digital beamforming, the UE can locate its own location based on RSS measurements of different sectors of beams. Let $\mathbf{s} \in \mathbb{R}^{N \times 1}$ 
be the observation vector from sector level sweeping which is given by

$$
\mathbf{s}=\mathbf{p}(\theta)+\mathbf{n}
$$

where $\mathbf{n} \in \mathbb{R}^{N \times 1} \sim \mathcal{N}\left(0, \sigma_{n}^{2} \mathbf{I}_{N}\right)$ is an additive white Gaussian noise vector, $\mathbf{p}(\boldsymbol{\theta})$ is the vector which contains the exact RSS information in logarithmic scale regarding location of the UE represented by $\theta \in \mathbb{R}^{2 \times 1}=[x, y]$. Unknown parameters $\theta_{1}$ and $\theta_{2}$ correspond to $x$ and $y$, respectively. The log-likelihood function of the UE's location is expressed as

$$
\mathcal{L}(\boldsymbol{\theta})=\log p(\mathbf{s} ; \boldsymbol{\theta})
$$

where likelihood function is given as

$$
p(\mathbf{s} ; \Theta)=\frac{1}{2 \pi \sigma_{\mathrm{n}}^{2}} \exp \left(-\frac{1}{2 \sigma_{\mathrm{n}}^{2}}(\mathbf{s}-\mathbf{p}(\theta))^{\mathrm{T}}(\mathbf{s}-\mathbf{p}(\theta))\right) .
$$

Maximum likelihood (ML) estimate of $\theta$ is formulated as

$$
\hat{\boldsymbol{\theta}}=\arg \max _{\boldsymbol{\theta}} \mathcal{L}(\mathbf{P} ; \hat{\boldsymbol{\theta}})
$$

which can be expressed as a nonlinear least squares (NLS) problem given by

$$
\hat{\boldsymbol{\theta}}=\arg \min _{\theta}\left(\|\mathbf{s}-\mathbf{p}(\theta)\|_{2}^{2}\right)
$$

The nonlinear optimization problem in 3.10 can be solved by Newton-Raphson method using the iterative approach 52

$$
\theta^{i+1}=\theta^{i}-\mathbf{H}^{\mathrm{T}}\left(\mathrm{s}-\mathrm{p}\left(\theta^{\mathrm{i}}\right)\right)
$$

where $\mathbf{H}$ represents Jacobian matrix of $\mathbf{p}(\boldsymbol{\theta})$ with respect to $\boldsymbol{\theta}$, and is explicitly given by

$$
\mathbf{H}=\left[\begin{array}{cccc}
\frac{\partial P_{11}}{\partial x} & \frac{\partial P_{21}}{\partial x} & \cdots & \frac{\partial P_{N N}}{\partial x} \\
\frac{\partial P_{11}}{\partial y} & \frac{\partial P_{21}}{\partial y} & \cdots & \frac{\partial P_{N N}}{\partial y}
\end{array}\right]^{\mathrm{T}}
$$




\subsection{Cramer-Rao Lower Bound}

The noise term in the (3.6) is assumed to be additive white Gaussian noise (AWGN), and it does not depend on the location of the UE. Therefore, Fisher Information Matrix (FIM) can be calculated as 52

$$
\mathbf{J}(\theta)=\frac{1}{\sigma_{n}^{2}} \mathbf{H}^{\mathbf{T}} \mathbf{H}
$$

Using $\mathbf{J}(\boldsymbol{\theta})$, CRLB of the Root Mean Square Error (RMSE) on the localization accuracy on $x y$-plane which is represented with $\mathrm{E}(x, y)$ can be acquired as

$$
\mathrm{E}(x, y) \geq \sqrt{\operatorname{tr}\left(\mathbf{J}^{-1}(\boldsymbol{\theta})\right)}
$$

Please note that CRLB is derived using (3.1)- 3.5$)$ and $(3.12)$, hence CRLB in $(3.14)$ is in generalized form, and can be derived for any $2 \mathrm{D}$ coordinates on $x y$-plane and arrangement of antenna array.

\subsection{Derivation of the CRLB}

In this section we will show derivation of CRLB through obtaining FIM. Using (3.1)(3.8), elements of Jacobian matrix of the observation vector, hence elements of FIM and CRLB, can be derived as follows. The partial derivative of 3.1 can be represented as

$$
\frac{\partial \hat{P}_{i}}{\partial \theta_{m}}=\frac{\partial\left(20 \log _{10}\left(\frac{P_{t} G_{r} \lambda^{2}}{16 L \pi^{2}}\right)\right)}{\partial \theta_{m}}+\frac{\partial\left(20 \log _{10} G_{t}\left(\phi_{i}\right)\right)}{\partial \theta_{m}}+\frac{\partial\left(20 \log _{10} \frac{1}{d_{b}^{2}}\right)}{\partial \theta_{m}} .
$$

The parameters $P_{t}, G_{r}, \lambda$, and $L$ do not depend on the location of the UE, and can be considered constants. Hence the resulting partial derivative of 3.1 is given by

$$
\frac{\partial \hat{P}_{i}}{\partial \theta_{m}}=\frac{20}{\ln 10}\left(\frac{\partial G_{t}\left(\phi_{i}\right)}{\partial \theta_{m}}+\frac{\partial\left(\frac{1}{d_{b}^{2}}\right)}{\partial \theta_{m}}\right) .
$$


For a special case of $\mathrm{d}_{\mathrm{s}}=\lambda / 2$ and assuming antenna efficiency $\varepsilon_{r}=1$, the gain function becomes

$$
G_{t}\left(\phi_{i}\right)=\frac{\sin \left(N \pi \cos \phi_{i}\right)}{\sin \left(\pi \cos \phi_{i}\right)}=\frac{\sin \left(\frac{N \pi x}{\sqrt{x^{2}+y^{2}}}\right)}{\sin \left(\frac{\pi x}{\sqrt{x^{2}+y^{2}}}\right)}
$$

First derivative of 3.17$)$ with respect to $\theta_{1}=x$ is given as

$$
\frac{\partial G_{t}\left(\phi_{i}\right)}{\partial x}=\frac{\pi y^{2}\left(N \cos \left(N \psi_{i}\right)-\cot \left(\psi_{i}\right) \sin \left(N \psi_{i}\right)\right)}{\sin \left(\psi_{i}\right)\left(x^{2}+y^{2}\right)^{3 / 2}}
$$

where $\psi_{i}=\mathrm{kd}_{\mathrm{s}} \cos \phi_{i}+\beta_{i}=\frac{\pi x}{\sqrt{x^{2}+y^{2}}}$. The same solution for $\theta_{2}=y$ is as follows

$$
\frac{\partial G_{t}\left(\phi_{i}\right)}{\partial y}=\frac{-\pi x y\left(N \cos \left(N \psi_{i}\right)-\cot \left(\psi_{i}\right) \sin \left(N \psi_{i}\right)\right)}{\sin \left(\psi_{i}\right)\left(x^{2}+y^{2}\right)^{3 / 2}}
$$

The path loss component does not vary with $\phi_{i}$, and it only depends on the distance between the BS and the UE. Then the derivative of the path loss component with respect to $\theta_{1}=x$ and $\theta_{2}=y$ is as follows

$$
\begin{aligned}
& \frac{\partial\left(x^{2}+y^{2}\right)^{-1}}{\partial x}=-\frac{2 x}{\left(x^{2}+y^{2}\right)^{2}}, \\
& \frac{\partial\left(x^{2}+y^{2}\right)^{-1}}{\partial y}=-\frac{2 y}{\left(x^{2}+y^{2}\right)^{2}} .
\end{aligned}
$$

Based on these derivations, using (3.15)-(3.21), the CRLB for any location can be calculated with known $\mathbf{x}_{\mathbf{b}}$ with given parameters $d_{\mathbf{s}}=\lambda / 2$.

\subsubsection{Asymptotic Behavior of CRLB}

In this subsection we will analyze asymptotic behavior of CRLB with respect to number of antenna elements. For very large $N$, the partial derivative of 3.16 becomes

$$
\frac{\partial \hat{P}_{i}}{\partial x}=\frac{\pi y^{2}\left(N \cos \left(N \psi_{i}\right)\right)}{\sin \left(\psi_{i}\right)\left(x^{2}+y^{2}\right)^{3 / 2}}
$$

for $\theta_{1}=x$, and for $\theta_{2}=y$ it becomes

$$
\frac{\partial \hat{P}_{i}}{\partial y}=\frac{-\pi x y\left(N \cos \left(N \psi_{i}\right)\right)}{\sin \left(\psi_{i}\right)\left(x^{2}+y^{2}\right)^{3 / 2}} .
$$


Table 3.1: Simulation parameters.

\begin{tabular}{|l|l|}
\hline Parameter & Value \\
\hline \hline Operating Frequency & $60 \mathrm{GHz}$ \\
\hline Transmit Power $\left(P_{t}\right)($ EIRP) & $30 \mathrm{dBm}$ \\
\hline Antenna Sensitivity & $-80 \mathrm{dBm}$ \\
\hline Room Width and Length & $8 \mathrm{~m} \mathrm{x} 8 \mathrm{~m}$ \\
\hline Granularity of Simulations & $1 \mathrm{~cm}$ \\
\hline Number of Antenna Elements $(\mathrm{N})$ & 32 \\
\hline Antenna Element Spacing $\left(d_{s}\right)$ & $\lambda / 2$ \\
\hline
\end{tabular}

Considering large $N$, the CRLB can be written as

$$
\mathrm{E}(x, y) \geq \frac{G(x, y)}{N^{2} \cos ^{2}\left(N \psi_{i}\right)},
$$

where $G(x, y)$ represents the portion of CRLB which has the parameters regarding to the location of the UE and not depending on $N$. The CRLB is inversely proportional to the square of number of antenna elements $\left(N^{2}\right)$.

\subsection{Numerical Results}

In this section, we evaluate the limits of accuracy of RSS-based localization and compare NLSE with CRLB using computer simulations. For simulation tractability, we consider an empty room of $8 \mathrm{~m} \times 8 \mathrm{~m}$ size. We consider a 32 element antenna array which is located at the center of the side wall, at the same height with the UE. Antenna elements are uniformly distributed on the array with distance $d_{s}=\lambda / 2$. The simulation parameters are given in Table 3.1 .

As in SLS, the beams are formed on discrete angles as virtual antenna sectors. They slice the half circle into number of excited antenna elements as in Fig. 3.2. A sample realization for $N=32$ is shown in Fig. 3.4. Maximum achievable RSS for all possible locations of UE is given in Fig. 3.4(a). There are no spots below -80 $\mathrm{dBm}$ since narrow and directional beams allow signal to reach further distances with 


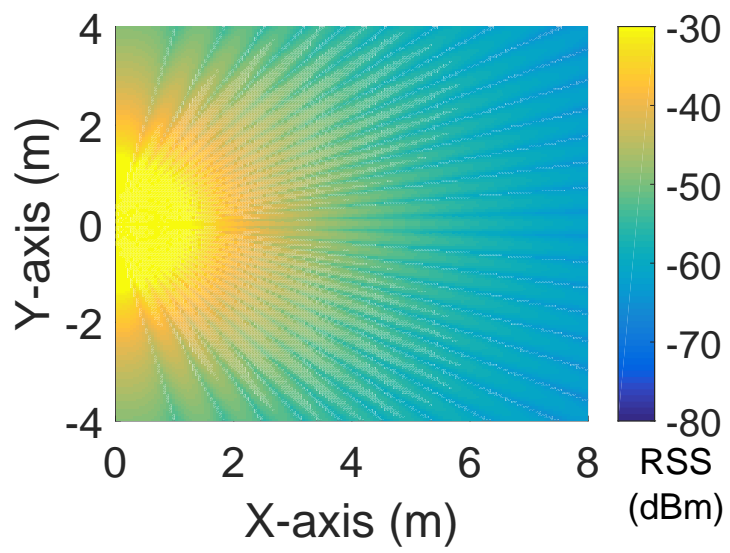

(a)

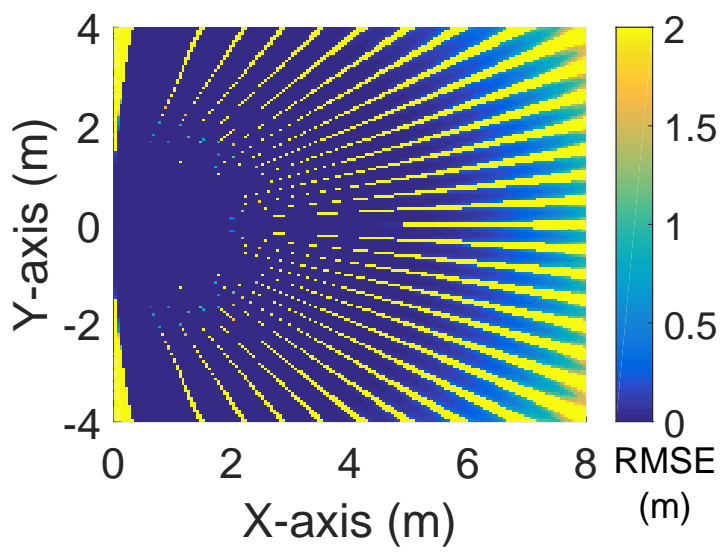

(b)

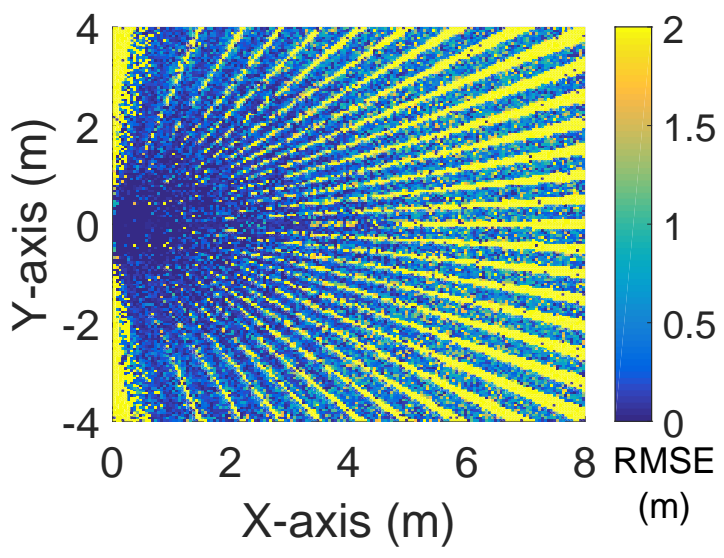

(c)

Figure 3.4: (a) Maximum achievable RSS at any possible UE location with 32 antenna elements at transmitter using SLS measurements. (b) CRLB for 32 antenna elements based on SLS measurements. (c) NLSE for 32 antenna elements based on SLS measurements. 


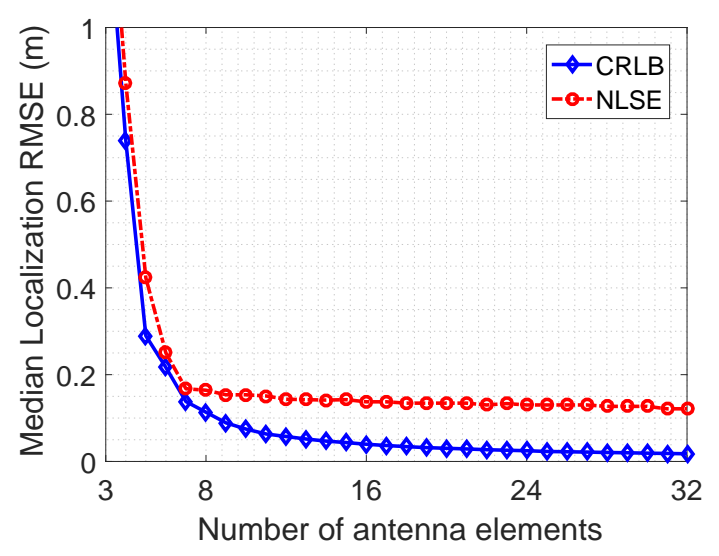

(a)

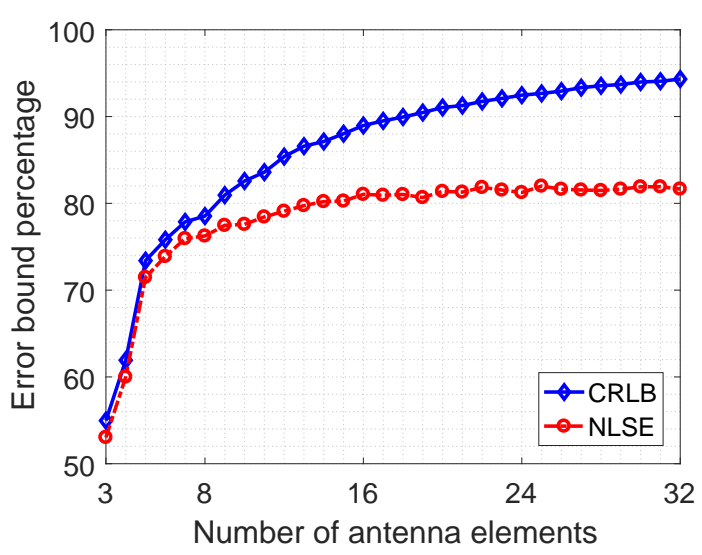

(b)

Figure 3.5: (a) Median Localization RMSE for various number of antenna elements. (b) 1 meter error bound percentage for various number of antenna elements.

less attenuation. However, due to non-overlapping beams there are blindspots even when for the locations closer than 2 meters to the BS. CRLB for the system with $N=32$ is shown in Fig. 3.4(b). The NLSE performance is given in Fig. 3.4(c) The performance of the NLSE is close to the CRLB for shorter distances from the BS. As the distance from the BS gets larger, the performance of the NLSE drops drastically compared to CRLB. This is mainly due to the antenna sensitivity, which causes to receive signals from only the antenna sectors directed towards the location of UE at further distances from the BS.

In Fig. 3.5, the localization accuracy is benchmarked over number of antenna elements $N$. In Fig. 3.5(a), median localization RMSE over defined system area is plotted versus number of antenna elements. The median localization RMSE is higher than 1 meter for both CRLB and NLSE for $N=3$. CRLB is inversely proportional to the square of number of antenna elements $N$ as shown in Section 3.5. As number of antenna elements increase, the median error for NLSE drops drastically, especially from $N=3$ to $N=8$. The median localization RMSE for $N=4$ is 0.87 meters for NLSE and 0.74 meters for CRLB. Increasing number of antenna elements to $N=8$ 


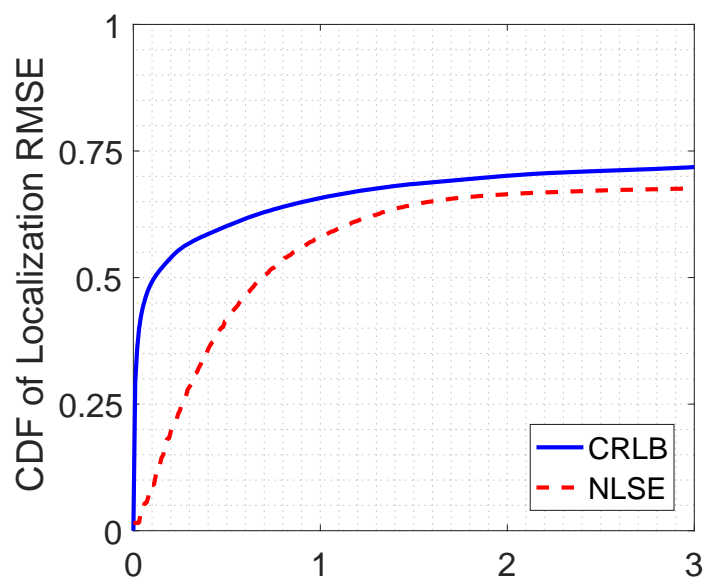

(a) Localization RMSE (m)

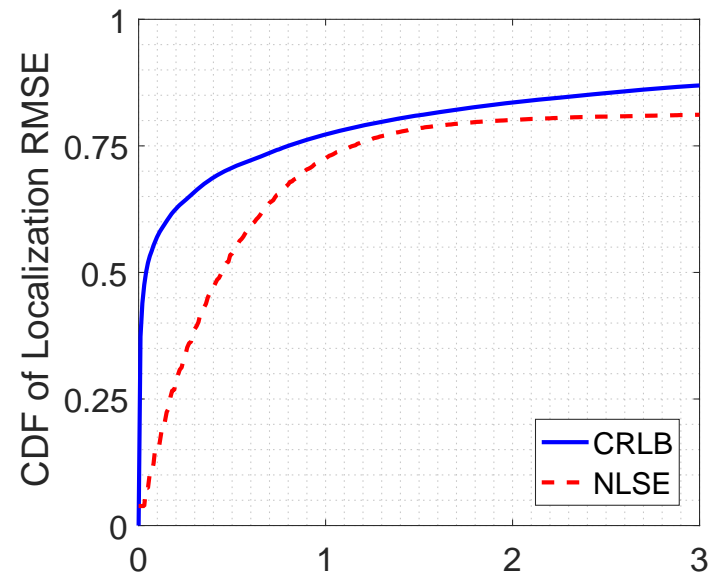

(b) Localization RMSE (m)

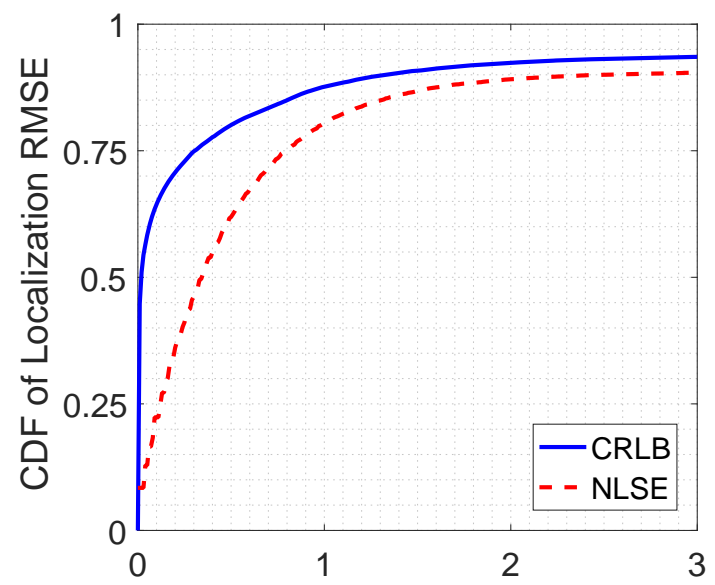

(c) Localization RMSE (m)

Figure 3.6: CDF of Localization RMSE for (a) $N=8$, (b) $N=16$, and (c) $N=32$. 
drops median localization RMSE to 0.17 meters for NLSE and to 0.11 meters for CRLB. Note that increasing the number of antenna elements after $N=8$ does not decrease median localization RMSE significantly since localization coverage reaches saturation.

Another performance metric for localization accuracy is error bound of 1 meter as shown in Fig. 3.5(b). In Fig. 3.5(b) the percentages of the areas with error of 1 meter or less in the system versus number of antenna elements are given for NLSE and CRLB. CRLB and NLSE are close to each other for lower number of antenna elements. Asymptotic behavior of CRLB and NLSE differ regarding number of antenna elements. As number of antenna elements increase, the separation between CRLB and NLSE gets larger. This is due to the bias of NLSE which is caused by local optimum cases due to nonconvex shape of radiation pattern (i.e. side lobes). CRLB is a lower bound for variance of unbiased estimator. CRLB and NLSE are around 55\% for $N=3$. NLSE saturates after $N=16$ around 80\%, while CRLB keeps increasing up to $93 \%$ for $N=32$.

CDF of localization RMSE is given in Fig. 3.6 for $N=8,16$ and 32 . The probability of localization error of 1 meter or below is 0.58 for $N=8,0.73$ for $N=16$, and 0.81 for $N=32$ with NLSE. The CDF of localization error of 1 meter or below is 0.65 for $N=8,0.76$ for $N=16$, and 0.84 for $N=32$ with CRLB. NLSE saturates around at a certain level, which means the localization coverage probability is 0.68 for $N=8$, while it is 0.81 for $N=16$, and 0.90 for $N=32$. Increasing number of antenna elements is critical for having less blindspots and better localization accuracy. However, due to the limitations of beamforming and beamshapes, increasing number of antenna elements becomes ineffective for enhancing the accuracy. 


\subsection{Conclusion}

In this chapter, fundamental limits on the localization accuracy of mmWave system is studied considering realistic radiation pattern of linear antenna array. Our results show that high accuracy of localization depends on number of antenna elements, however accuracy saturates at a certain number due to limitations of beamforming and angles. In our simulations, NLSE diverges from CRLB as number of antenna elements increases since side lobes become an interferer at further distances where number of measurements are limited. 


\section{CHAPTER 4 \\ OCCUPANCY COUNTING USING PROBE REQUESTS}

Smart cities of the future are expected to provide better use of public resources, increase quality of service offered to citizens, and reduce operational costs of public administrators [6]. Internet of Things (IoT) technology is a key enabler for smart cities, and it can support a plethora of services, ranging from building health inspection, to waste management, noise/air quality monitoring, traffic congestion control, city energy consumption reduction, smart parking/lighting, and automation of smart buildings 7 . . Realizing the vision of smart cities necessitate effective use of IoT technologies for proximity detection, localization, tracking of objects and humans, and occupancy monitoring 64, 65].

Smart buildings constitute a key component of smart cities, which will benefit extensively from the use of IoT technologies for health monitoring, energy management, public safety, and surveillance, [66]. In particular, buildings are among the largest consumers of electricity in the United States: they account for $40 \%$ of primary energy consumption and $72 \%$ of electricity consumption [6]. An important portion of the electricity consumption of buildings is used for heating, ventilation, and air conditioning (HVAC). To this end, low cost, seamless, and accurate occupancy counting can help in achieving significant energy savings in smart buildings, such as by dynamically scheduling HVAC activity based on real-time building occupancy levels at different areas [10].

Occupancy counting in smart buildings can be implemented via video processing and camera systems or deployment of occupancy sensors throughout the building [67]. These options require installation of new equipment that are often costly to deploy. An alternative way is to use ambient wireless signals of opportunity that uniquely match to building occupants. While other technologies such as sensors, cameras, 


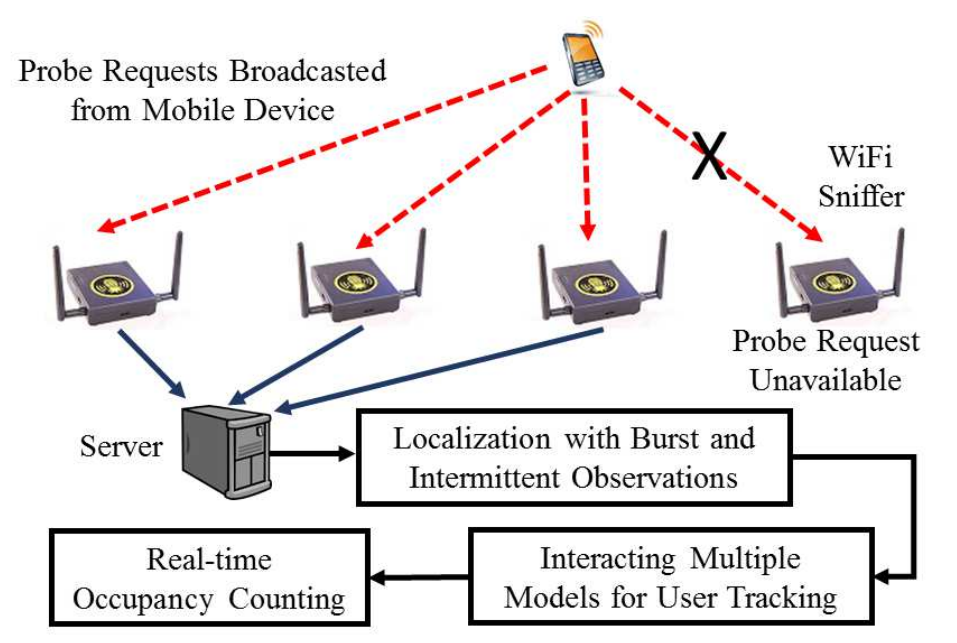

(a)

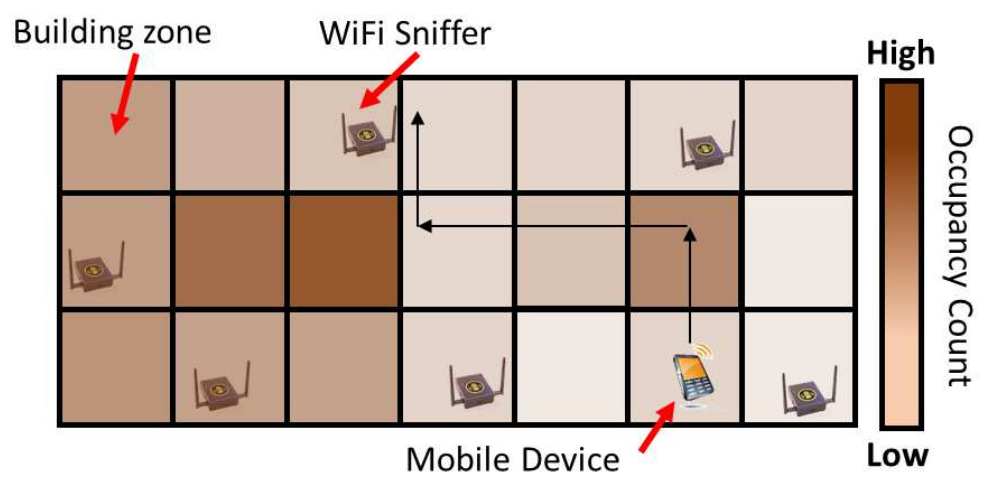

(b)

Figure 4.1: Occupancy counting using probe requests: (a) Capturing burst and intermittent probe requests at multiple WiFi Pineapples; (b) Zone-based real-time occupancy counting.

and RFID provide occupancy tracking at certain building zones, WiFi technology is already available in most buildings, and can provide good occupancy monitoring coverage. Even though several positioning solutions exist based on available WiFi infrastructure, they typically require a connection between user equipment and WiFi access points (APs) 67 68.

In this work, we consider the use passive sniffing of WiFi probe requests for occupancy monitoring and tracking in smart buildings as illustrated in Fig. 4.1. Probe requests are signals that are continuously broadcast from devices with WiFi tech- 
nology, such as smartphones, laptops, and tablets [69 73]. The probe requests are not encrypted, and can be captured and decoded with the help of passive sniffers as shown in Fig. 4.1(a), without connecting to a particular network. Probe requests are also burst in nature, since they are broadcasted in the air in search of WiFi networks to get connected, to get a list of available networks, or to handover between $\mathrm{WiFi}$ APs. Frequent transmission of probe requests from mobile devices introduces an opportunity to track the occupancy count in different zones of a building by simply monitoring the probe requests (see Fig. 4.1(b)). In particular, we can capture the received signal strength (RSS) of probe requests using sniffers such as WiFi Pineapple (WiFi-PA) [74], which can then be used for occupancy monitoring inside the building.

To the best knowledge of the authors, there are no detailed studies in the literature that report efficiency of occupancy counting using WiFi probe requests using varying number of reference nodes to track individual devices. In this work, we use WiFi probe requests captured at various reference locations for occupancy monitoring in smart buildings as summarized in Fig. 4.1. To this end, seven WiFi-PAs are deployed at various locations within the FIU Engineering Center, and probe requests are collected over multiple days. The burst and intermittent nature of probe requests require post processing of the data to make it ready for the localization and tracking. Subsequently, at every sampling window, various localization techniques are used to obtain location estimates of each WiFi device, which are further refined using an Interacting Multiple Model (IMM) filter for tracking user location. Position estimates are then aggregated into one of the eight occupancy zones inside the building for real-time occupancy counting.

The main novel contributions of this work can be summarized as follows:

- We propose an adaptive tracking algorithm, which enables to study occupancy count with burst and intermittent measurements, as well as the varying number 
of positioning reference nodes (sniffers) during location tracking. To authors' best knowledge, there is no similar study considering both conditions in the literature.

- We develop estimators and heuristic methods to localize and track the target node even with measurements from a single reference node in the worst case. In our experiments, we observe that $47.3 \%$ of the time, probe requests are detected by a single reference node whereas $36.9 \%$ of them are received by two reference nodes. Thus, including them in tracking and occupancy counting is crucial for robustly estimating occupancy count in building zones.

- We implement simulations with realistic WiFi channel models to show the occupancy detection performance of our proposed framework. We also show the accuracy of our approach using real world data, by carrying out an experiment with WiFi-PAs as the probe request sniffers for occupancy tracking for an indoor university campus environment. Proposed method achieves up to $90 \%$ performance in zone-level tracking. Our simulation results agree with experimental results, which show close performance.

This chapter is organized as follows. In Section 4.1, a brief overview of the existing literature related to the tracking techniques is presented. Models for the tracking algorithm are explained in Section 4.2. Localization and initialization for user tracking using probe request data are presented in Section 4.3, while tracking with the IMM filters and zone-level occupancy counting are provided in Section 4.4. Subsequently, gathering of probe requests is explained in Section 4.5 in detail. Simulation and experimental results are presented in Section 4.6, and finally, concluding remarks and future prospects are summarized in Section 4.7 . 


\subsection{Literature Review}

Use of probe requests have recently received interest from researchers for various applications. For example, they are used for load balancing in wireless networks to find hidden and mobile nodes in [75], and to analyze the handover processes of 802.11 network in $[76]$. A WiFi flood attack detection system, which is a method based on the probe request and probe response timeouts, is proposed in [77]. Privacy issues with probe requests are evaluated in [78], where authors utilize probe requests to link the devices by creating a table for the requested WiFi network names and comparing them with the other devices.

Although RSS-based tracking with Kalman filters (KF) is a well-established area, it is still an interesting research topic due to its various new applications for accurate localization [79,80]. In [81 two-slope RSS model is used with two Extended Kalman Filters (EKF) considering an IMM framework to improve tracking accuracy. In [82], the authors present a novel exponential-Rayleigh RSS model for device-free localization and tracking with KF, where the main contribution is to include multipath components in RSS model for improving localization and tracking accuracy. The accuracy is shown to increase significantly compared to the standard RSS model. In 83], the RSS variance problem in tracking due to the hardware differences, device placement, and environmental changes are studied, and a particle filter based solution is proposed. The results show that the tracking accuracy is more robust against the RSS variance when the accelerometer and digital compass readings are included in system. In [84], a novel approach based on compressive sensing is used for the localization and tracking of WiFi devices. In particular, coarse estimates of RSS fingerprinting are improved by the compressive sensing techniques, and KF with map information is used to track the devices. 
In the papers mentioned above, a constant number of reference nodes are always assumed to continuously localize and track the users. However, in practice, reference nodes may fail to detect transmitted signal or may not be available at all times. Even though there are several studies suggesting increased localization and tracking accuracy by introducing secondary reference nodes based on their location estimates in cooperative networks [85], or self-adaptive localization techniques with a similar approach [86], they have not investigated localization with the limited and dynamically changing number of the reference nodes.

\subsection{System Model}

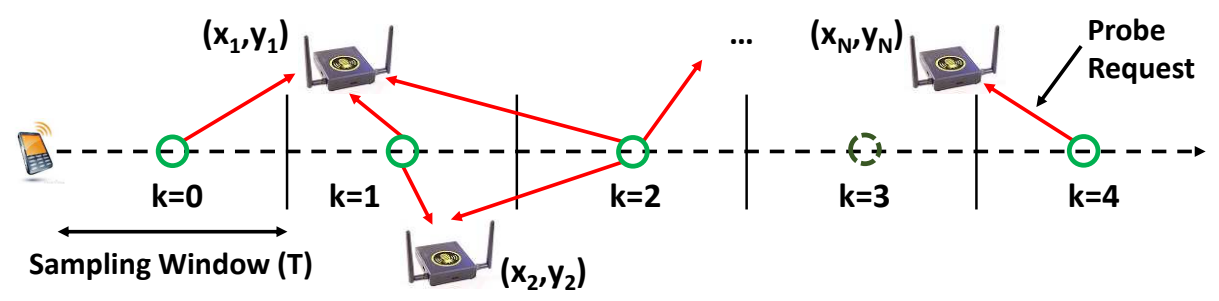

Figure 4.2: Tracking by using varying number of reference nodes.

In this work, we consider an occupancy counting scenario as seen in Fig. 4.2, There are $N$ reference nodes $\mathcal{R}=\left\{r_{1}, \cdots, r_{N}\right\}$ placed through the tracking zone. The known location of $i$ th reference node is denoted with $\boldsymbol{x}_{i}=\left(x_{i}, y_{i}\right)$, and a target node has an unknown position $\boldsymbol{x}^{\prime}=\left(x^{\prime}, y^{\prime}\right)$. Burst and intermittent probe requests are broadcasted from the mobile target node as shown in Fig. 4.3. We consider a predefined sampling window, where data from all the burst probe requests within the window are aggregated. In particular, the $k$ th sampling window of duration $T$ spans the interval between time instances between $t_{k}$ and $t_{k-1}$. Eventually, the true location of the target node in the sampling window $k$ is represented with $\boldsymbol{x}^{\prime}{ }_{k}=\left(x_{k}^{\prime}, y_{k}^{\prime}\right)$. 
During tracking of the mobile target node, some reference nodes may not detect transmitted probe requests by the target node due to being far away from the target, poor link quality, and collision of probe request packets, as illustrated in Fig. 4.2. In such cases, we need to be able to work with RSS from the rest of the reference nodes, which are called available measurements in sampling window $k$, and denoted by a set $\mathcal{P}_{k}=\left\{p_{i, k}, \ldots, p_{u, k}\right\}$, where $p_{i, k}$ represents RSS value at reference node $i$ at sampling window $k$.

We refer $p_{i, k}$ as a representative RSS value for window $k$ at node $i$, since there might be multiple probe requests observed from a certain target node within the same sampling window as seen in Fig. 4.3. Then, we have to extract the representative RSS value from these burst of observations. Even though the RSS values are usually close or equal to each other for spatio-temporally close probe requests, there might be outliers due to the varying channel conditions. Using the mean of the RSS values would therefore bias our results towards outliers; therefore, median value of the RSS values within the sampling window is used as a representative RSS for that sampling window as follows

$$
p_{i, k}=\operatorname{median}\left\{p_{i, k, 1}, \ldots, p_{i, k, m_{i, k}}\right\}
$$

where the $i$ th reference node is considered to receive $m_{i, k}$ different probe requests within the sampling window $k$. Let the RSS measurement for the probe request with the median RSS value be defined as

$$
p_{i, k}=P_{0}-10 n \log _{10}\left(\frac{d_{i, k}}{d_{0}}\right)+w
$$

where $P_{0}$ is the signal strength at the reference distance $d_{0}, n$ is the path loss exponent (PLE), $d_{i, k}=\left\|\boldsymbol{x}_{k}^{\prime}-\boldsymbol{x}_{i}\right\|$ is the Euclidian distance between the $i$ th reference node and the target device at sampling window $k$, and $w \sim \mathcal{N}\left(0, \sigma^{2}\right)$ is the noise modeled by additive white Gaussian noise (AWGN) with a variance of $\sigma^{2}$ and mean of zero. 


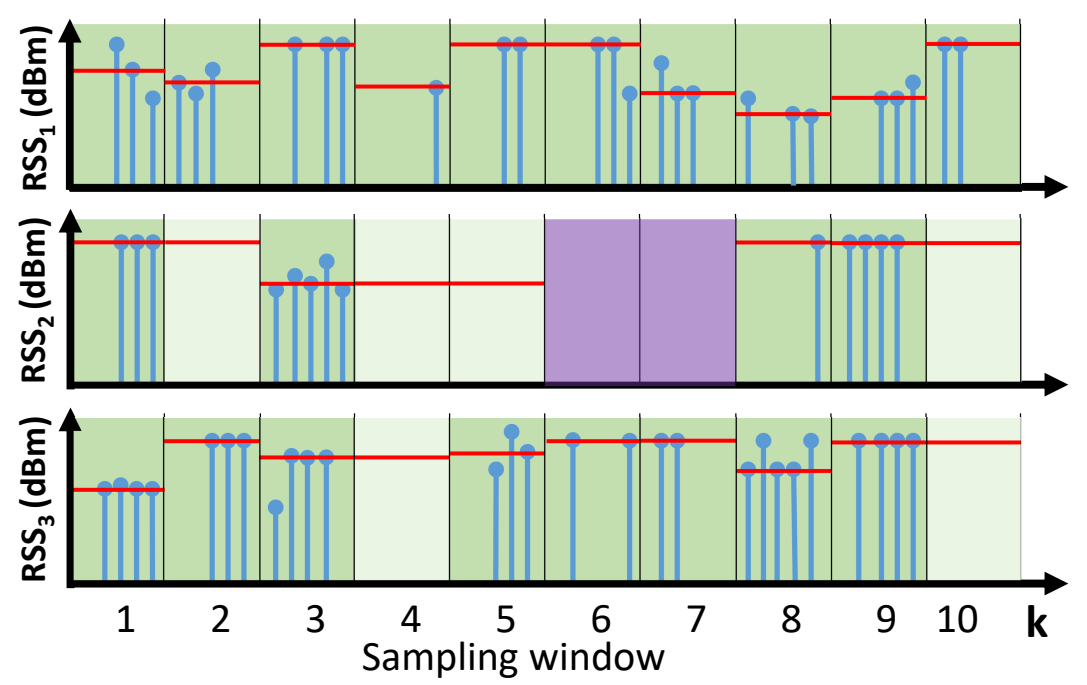

Figure 4.3: Burst and intermittent probe requests from a mobile device, observed at three different WiFi-PAs within different sampling windows, with sample-and-hold window duration $L=2$.

Once the representative RSS measurements are obtained for each reference node $i$ within the $k$ th sampling window, the number of the available representative RSS measurements from the different reference nodes at sampling window $k$ is given by $\left|\mathcal{P}_{k}\right|$, which is the cardinality of set $\mathcal{P}_{k}$. Due to the intermittent nature of probe requests that may result in unavailable measurements within subsequent measurement windows, a sample-and-hold approach is used to hold RSS values for a certain number of sampling windows at different reference nodes. Otherwise, the useful ranging information from the difference nodes would be lost. In Fig. 4.3, an example sampling windows is given with $L=2$, where $L$ is the holding length.

Assume that the last probe request detected by the $i$ th reference node is within the sampling window $k$, and the $i$ th reference node did not receive any probe requests within future sampling window(s). Then, the RSS value of the $i$ th reference node for the samples after the sampling window $k$ using a holding length $L$ can be written as

$$
p_{i, k+l}=\left\{\begin{array}{ll}
p_{i, k+l}, & \text { if } m_{i, k+l} \geq 1 \\
p_{i, k}, & \text { if } \sum_{s=1}^{l} m_{i, k+s}=0
\end{array},\right.
$$


for $l=1, \cdots, L$, given $m_{i, k} \geq 1$. If there is still no available signal received at the $i$ th reference node after the sample-and-hold window, the RSS measurement will be removed from the set of the available RSS values $\mathcal{P}_{k}$ which represented with purple color in Fig. 4.3 .

Given this system model and framework, our main goal in this work is to determine zone occupancy based on tracking position of WiFi devices with varying number of the available reference nodes and intermittent transmission of the probe requests. In the following sections, we explain the localization of the individual target nodes, IMMbased tracking with varying conditions, and finally zone-level mapping and occupancy counting of WiFi devices.

\subsection{Localization and Initialization Techniques}

In this section, RSS-based localization of the target node with varying number of reference nodes and different levels of a priori information will be presented. The technique used for localization changes with the number of available reference nodes and the unknown parameters is shown in Table 4.1. Since the unknown parameters are revealed in time, we will use them in the following sampling windows as prior information. The novelty of our system lies in the use of any number of reference nodes without discarding any single probe request.

Below, we will first consider heuristics and algorithms for estimating target location when we do not know the prior location information of the target (e.g., the target enters a building and no prior measurements available). Subsequently, in Section 4.4 we will investigate how we can improve the localization accuracy when the prior trajectory of the target is available. 


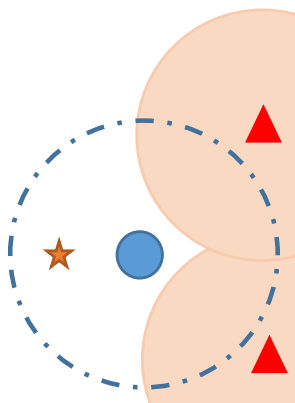

(a)

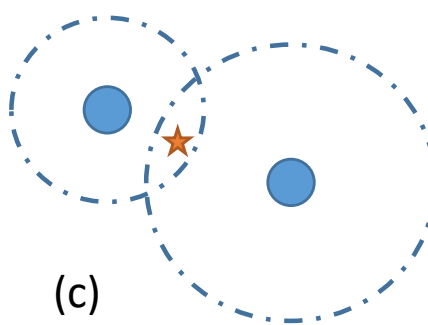

(d)
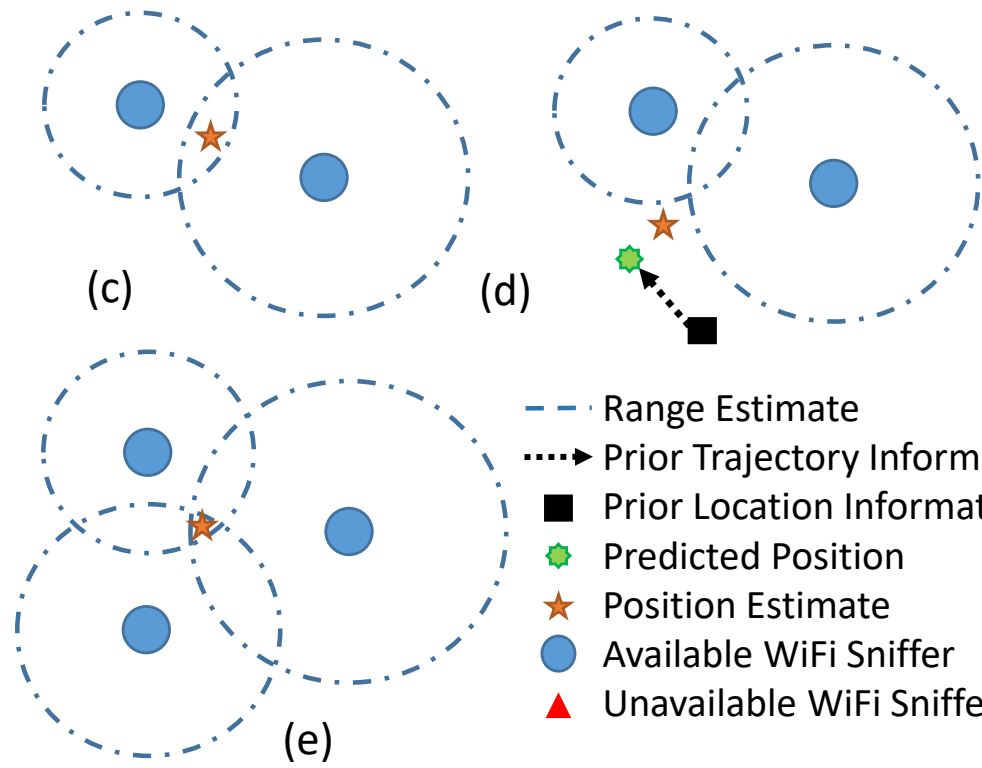

- - Range Estimate

.... Prior Trajectory Information

- Prior Location Information

Predicted Position

$\star$ Position Estimate

Available WiFi Sniffer

- Unavailable WiFi Sniffer

Figure 4.4: Tracking scenarios under the different circumstances, (a) initialization with $\left|\mathcal{P}_{k}\right|=1$ and (b) Model-1, (c) initialization with $\left|\mathcal{P}_{k}\right|=2$ and (d) Model-2, and (e) both Initialization with $\left|\mathcal{P}_{k}\right| \geq 3$ and Model-3.

\subsubsection{RSS Available at Three or Less Reference Nodes}

First, consider the case that the RSS measurement in the sampling window $k$ is available at only a single reference node (i.e. when $\left|\mathcal{P}_{k}\right|=1$ ), and $P_{0}$ and $n$ are unknown. Since $P_{0}$ and $n$ can only be estimated when $\left|\mathcal{P}_{k}\right| \geq 4$ (see Section 4.3.2), we consider that (as in $87 \mid$ ) $P_{0}$ and $n$ are obtained by averaging over (potentially limited number of) ground truth measurements where the location of the target is known. 
Table 4.1: Tracking models under various circumstances.

\begin{tabular}{|c|c|c|}
\hline & No Bayesian Tracking & Bayesian Tracking \\
\hline$\left|\mathcal{P}_{k}\right|=1$ & Heuristic-1 & Model-1 $(j=1)$ \\
\hline$\left|\mathcal{P}_{k}\right|=2$ & Heuristic-2 & Model-2 $(j=2)$ \\
\hline$\left|\mathcal{P}_{k}\right|=3$ & Heuristic-3 & Model-3 $(j=3)$ \\
\hline$\left|\mathcal{P}_{k}\right| \geq 4$ & NLS & Model-3 $(j=3)$ \\
\hline
\end{tabular}

Then, the target device is assumed to be at the vicinity of the available reference node, and we may estimate the location of the target device to be

$$
\hat{\boldsymbol{x}}^{\prime}{ }_{k}=\operatorname{median}\left\{\mathcal{S}_{u}-\cup_{u=1}^{N} \mathcal{S}_{u} \mid u \neq i\right\}
$$

where $\mathcal{S}_{i}$ is the set of possible locations in the coverage of $i$ th reference node, considering a circular coverage area for all reference nodes. In other words, (4.4) implies that the location is estimated to be in the median location of possible positions sampled densely within the coverage area of the reference node. On the other hand, as shown in Fig. 4.4(a), coverage area of several reference nodes may overlap, and the location of the target node is assumed not to be within the coverage area of the reference nodes that do not have the RSS measurement from the target node. Even though the location estimate in (4.4) is relatively coarse and based solely on the coverage area of the reference nodes, it is acceptable for the zone-level estimation, and we can still utilize the probe request measurements even if they are available only at a single reference node. It also allows to initialize the IMM tracking algorithm in Section 4.4 at a better location for quicker and more accurate estimation, and to avoid local optimums.

Second, consider that the RSS information from the target node at sampling window $k$ is available only at two reference nodes $\left(\left|\mathcal{P}_{k}\right|=2\right)$ and no prior information is available on the location of the target node. Then, the location estimate $\hat{\boldsymbol{x}}_{k}$ for the 
sampling window $k$ can be estimated as follows:

$$
\hat{\boldsymbol{x}}_{k}^{\prime}=\boldsymbol{x}_{i}+\left(\hat{d}_{i, k}+\frac{\hat{d}_{i, k}+\hat{d}_{u, k}-d_{i u}}{2}\right) \frac{\boldsymbol{x}_{u}-\boldsymbol{x}_{i}}{\left\|\boldsymbol{x}_{u}-\boldsymbol{x}_{i}\right\|},
$$

where $d_{i j}$ represents the distance between the $i$ th and $u$ th reference nodes, and the estimated distance between the $i$ th reference node and the target node $\left(\hat{d}_{i, k}\right)$ based on the RSS measurements is given as

$$
\hat{d}_{i, k}=10^{\frac{P_{0}-p_{i, k}}{10 n}}
$$

In other words, as shown in Fig. 4.4(c), the target node is assumed to be on the straight line that passes through the locations of the two reference nodes, and positioned on the line depending on the RSS measurements at each node.

Third, consider that at sampling windows $k$, RSS measurements are available at three or more reference nodes $\left(\left|\mathcal{P}_{k}\right| \geq 3\right)$. Then, we consider a linear least squares (LLS) solution as a low computational complexity method [26,88 that has acceptable localization performance as shown in Fig. 4.4(e). The location of the target device can be obtained by solving the LLS problem in the form $\mathbf{M} \boldsymbol{x}_{k}=\mathbf{b}$ with

$$
\begin{gathered}
\mathbf{M}=\left[\begin{array}{cc}
x_{1}-\frac{1}{N} \sum_{i=1}^{N} x_{i} & y_{1}-\frac{1}{N} \sum_{i=1}^{N} y_{i} \\
\vdots & \vdots \\
x_{N}-\frac{1}{N} \sum_{i=1}^{N} x_{i} & y_{N}-\frac{1}{N} \sum_{i=1}^{N} y_{i}
\end{array}\right], \\
\mathrm{b}=\frac{1}{2}\left[\begin{array}{c}
\left(x_{1}^{2}-\frac{1}{N} \sum_{i=1}^{N} x_{i}^{2}\right)+\left(y_{1}^{2}-\frac{1}{N} \sum_{i=1}^{N} y_{i}^{2}\right) \\
-\left(\hat{d}_{1, k}^{2}-\frac{1}{N} \sum_{i=1}^{N} \hat{d}_{i, k}^{2}\right) \\
\vdots \\
\left(x_{N}^{2}-\frac{1}{N} \sum_{i=1}^{N} x_{i}^{2}\right)+\left(y_{N}^{2}-\frac{1}{N} \sum_{i=1}^{N} y_{i}^{2}\right) \\
-\left(\hat{d}_{N, k}^{2}-\frac{1}{N} \sum_{i=1}^{N} \hat{d}_{i, k}^{2}\right)
\end{array}\right],
\end{gathered}
$$

where $\mathbf{M}$ and $\mathbf{b}$ are defined with the known position of the reference nodes $\left(x_{i}, y_{i}\right)$ and ranging information for reference nodes using (4.6). The solution to the LLS 
problem can then be found by solving for $\boldsymbol{x}_{k}{ }_{k}$, as follows:

$$
{\hat{\boldsymbol{x}^{\prime}}}_{k}=\left(\mathbf{M}^{T} \mathbf{M}\right)^{-1} \mathbf{M}^{T} \mathbf{b} .
$$

\subsubsection{RSS Available at Four or More Reference Nodes}

Consider now that the RSS information is available at least at four reference nodes,

i.e., $\left|\mathcal{P}_{k}\right| \geq 4$. Then, adapting [89], a nonlinear least squares algorithm is used to solve the localization problem with unknown parameters. The optimization problem is based on the relaxation of a maximum likelihood estimator with Taylor expansions of RSS defined in (4.2), around assumed values of unknown parameters [89]. The general optimization problem is defined as a special type of quadratic problem, general trust region problem, given as follows

$$
\begin{aligned}
\underset{\boldsymbol{y}_{v}}{\operatorname{minimize}} & \left\|\mathbf{Z}_{v} \boldsymbol{y}_{v}-\boldsymbol{b}_{v}\right\|^{2} \\
\text { subject to } & \boldsymbol{y}_{v}^{T} \boldsymbol{D}_{v} \boldsymbol{y}_{v}+2 \boldsymbol{f}_{v}^{T} \boldsymbol{y}_{v}=0,
\end{aligned}
$$

where $\mathbf{Z}_{v}$ is the translation matrix and $\boldsymbol{y}_{v}$ is the vector for unknown parameters and their derivations for case $v$, and $\boldsymbol{b}_{v}$ holds the measurements regarding the unknown parameters. The constraint $\boldsymbol{y}_{i}^{T} \boldsymbol{D}_{i} \boldsymbol{y}_{i}+2 \boldsymbol{f}_{i}^{T} \boldsymbol{y}_{i}=0$ defines the feasible set for solution of optimization, where $\boldsymbol{D}_{i}$ and $\boldsymbol{f}_{i}$ are the selection diagonal and vector, respectively. Each of these vectors and matrices are defined and given explicitly for three different availability level of a priori information below (i.e., estimated or unknown $P_{0}$ and $n$ ).

Unknown $P_{0}(v=1)$ In the case of unknown $P_{0}$ and $\boldsymbol{x}_{k}^{\prime}$ with knowledge of $n$, at least three reference nodes are required to estimate $P_{0}$. If the number of the reference nodes are less than three, approaches introduced in Section 4.3.1 are used. 
The optimization problem given in 4.10 for this case is written as

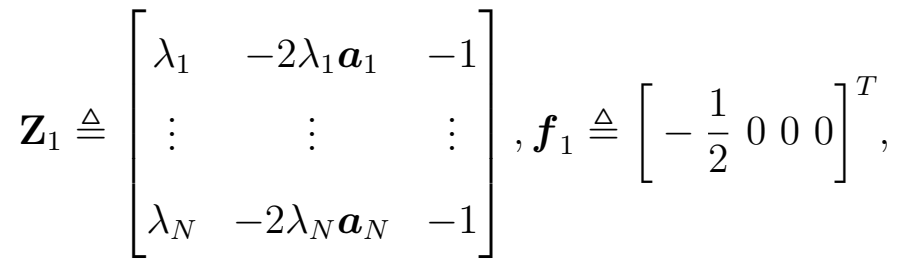

$$
\begin{aligned}
& \boldsymbol{b}_{1} \triangleq\left[-\lambda_{1}\left\|\boldsymbol{a}_{1}\right\|^{2}, \cdots,-\lambda_{N}\left\|\boldsymbol{a}_{N}\right\|^{2}\right]^{T}, \boldsymbol{D}_{1} \triangleq \operatorname{diag}(0,1,1,0) \text {, }
\end{aligned}
$$

where $\lambda_{i} \triangleq 10^{p_{i} /(5 n)}$ and $\alpha=10^{P_{0} /(5 n)}$, and solution for minimization problem is defined as $\boldsymbol{y}_{1} \triangleq\left[\left\|\boldsymbol{x}_{k}^{\prime}\right\|^{2} \boldsymbol{x}_{k}^{\prime T} \alpha\right]$.

Unknown $n(v=2)$ In the case of unknown $n$ and $\boldsymbol{x}_{k}^{\prime}$ with knowledge of $P_{0}$, optimization matrices and vectors become

$$
\begin{aligned}
& \mathbf{Z}_{2} \triangleq\left[\begin{array}{ccc}
1 & -2 \boldsymbol{a}_{1} & q_{1} \ln q_{1} \\
\vdots & \vdots & \vdots \\
1 & -2 \boldsymbol{a}_{N} & q_{N} \ln q_{N}
\end{array}\right], \boldsymbol{f}_{2} \triangleq\left[\begin{array}{cccc}
-\frac{1}{2} & 0 & 0 & 0
\end{array}\right]^{T}, \\
& \boldsymbol{b}_{2} \triangleq\left[q_{1}-\left\|\boldsymbol{a}_{1}\right\|^{2}, \cdots, q_{N}-\left\|\boldsymbol{a}_{N}\right\|^{2}\right]^{T}, \boldsymbol{D}_{2} \triangleq \operatorname{diag}(0,1,1,0) \text {, }
\end{aligned}
$$

where $q_{i} \triangleq 10^{\left(P_{0}-p_{i, k}\right) /\left(5 n_{0}\right)}$ and $n_{0}$ is the tuning parameter of the algorithm for $n$. The final solution of the optimization is defined as $\boldsymbol{y}_{2} \triangleq\left[\left\|\boldsymbol{x}_{k}^{\prime}\right\| \boldsymbol{x}_{k}^{\prime T} \delta\right]$ where $\delta \triangleq \frac{\left(n-n_{0}\right)}{n_{0}}$ shows the suitability of the tuning parameter.

Unknown $P_{0}$ and $n(v=3) \quad$ In the worst case, which there is no prior information on the parameters, we have to estimate both the location of the target nodes and channel parameters with below matrices and vectors

$$
\begin{aligned}
\mathbf{Z}_{3} \triangleq\left[\begin{array}{cccc}
1 & -2 \boldsymbol{a}_{1} & -g_{1} & g_{1} \ln g_{1} \\
\vdots & \vdots & \vdots & \vdots \\
1 & -2 \boldsymbol{a}_{N} & -g_{N} & g_{N} \ln g_{N}
\end{array}\right], \boldsymbol{b}_{3} \triangleq\left[-\left\|\boldsymbol{a}_{1}\right\|^{2}, \cdots,-\left\|\boldsymbol{a}_{N}\right\|^{2}\right]^{T} \\
\boldsymbol{f}_{3} \triangleq\left[\begin{array}{cccc}
-\frac{1}{2} & 0 & 0 & 0
\end{array}\right]^{T}, \boldsymbol{D}_{3} \triangleq \operatorname{diag}(0,1,1,0,0)
\end{aligned}
$$


where $g_{i} \triangleq 10^{\left(\bar{P}_{0}-p_{i, k}\right) /\left(5 n_{0}\right)}$ and $\bar{P}_{0}$ is the tuning parameter for $P_{0}$. Solution for the corresponding NLS to a general trust region problem is defined as $y_{3} \triangleq\left[\left\|\boldsymbol{x}_{k}^{\prime}\right\|^{2} \boldsymbol{x}_{k}^{\prime T} \gamma \gamma \delta\right]^{T}$ where $\gamma \triangleq 10^{\left(P_{0}-\bar{P}_{0}\right) /(5 n)}$ is a measure on $P_{0}$ tuning parameter suitability. The IMM based tracking takes the localization estimates as an input, which is explained in the following section in details.

\subsection{IMM Tracking and Occupancy Counting}

In this section, we study Bayesian tracking of the individual WiFi devices by exploiting prior location information of the mobile device. Probe requests are intermittent, which means that the target device may not send any probe signals over a duration of many sampling windows. Even when the probe requests are transmitted by the mobile device, they may not always be captured at the reference node. Therefore, using a standard Kalman filtering technique (see e.g. [90, 91]) that assumes uniform set of system parameters will not work effectively. Considering varying system properties such as the number of the available measurements and prior information related to the device, we consider an IMM framework that employs multiple, interacting Kalman filters in this study. The overall model 92 is represented with a linear system as follows

$$
\begin{aligned}
& \mathbf{x}_{k}=\mathbf{A}_{k-1}^{j} \mathbf{x}_{k-1}+\mathbf{q}_{k-1}^{j} \\
& \mathbf{y}_{k}=\mathbf{H}_{k}^{j} \mathbf{x}_{k}+\mathbf{r}_{k}^{j},
\end{aligned}
$$

where $\mathbf{x}_{k} \in \mathbb{R}^{n}$ is the state of the system during sampling window $k, \mathbf{A}_{k-1}^{j}$ is the transition matrix for the model order $j$, where the model order depends on the number of available reference nodes (see Table 4.1). which is in effect during the sampling windows $k-1$, and $\mathbf{q}_{k-1}^{j} \sim \mathcal{N}\left(\mathbf{0}, \mathbf{Q}_{k-1}^{j}\right)$ is the process noise which is Gaussian distributed with zero mean and covariance $\mathbf{Q}_{k-1}^{j}$ for the $j$ th model. The measurement on the 
sampling window $k$ is denoted by $\mathbf{y}_{k} \in \mathbb{R}^{m}$, where $\mathbf{H}_{k}^{j}$ is the $j$ th measurement model matrix, and $\mathbf{r}_{k}^{j} \sim \mathcal{N}\left(\mathbf{0}, \mathbf{R}_{k}^{j}\right)$ is the measurement noise on the step $k$ for $j$ th model with Gaussian distribution of mean zero and covariance $\mathbf{R}_{k}^{j}$. As explained earlier, each model in the system has its own Kalman filter parameters, and the resulting estimate is calculated with the selection of the correct model based on the status of the system such as $\left|\mathcal{P}_{k}\right|$ and prior information of channel parameters.

A standard Wiener process velocity model is used for all of the models, with varying measurement noise $\left(\mathbf{r}_{k}^{j}\right)$ and step sizes based on sampling window length $\left(\Delta t_{k}=T\right)$. Defining matrices of the models are given as

$$
\mathbf{A}_{k}^{j}=\left[\begin{array}{cccc}
1 & 0 & \Delta t_{k} & 0 \\
0 & 1 & 0 & \Delta t_{k} \\
0 & 0 & 1 & 0 \\
0 & 0 & 0 & 1
\end{array}\right], \mathbf{H}_{k}^{j}=\left[\begin{array}{cccc}
1 & 0 & 0 & 0 \\
0 & 1 & 0 & 0
\end{array}\right] .
$$

\subsubsection{Review of a Generic Kalman Filter}

Kalman filtering, in general, has two steps: 1) prediction and 2) update. In the prediction step, next state of the system is predicted with the given previous measurements, and in the update step, the current state of the system is estimated with the given measurement at that sampling window [92]. In below, first the general procedure for the prediction and update stages are mathematically summarized (model index $j$ will be dropped for brevity), and we will subsequently discuss how Kalman filters with multiple models can be utilized for our scenario.

\section{Prediction}

$$
\begin{aligned}
& \mathbf{m}_{k}^{-}=\mathbf{A}_{k-1} \mathbf{m}_{k-1}, \\
& \mathbf{U}_{k}^{-}=\mathbf{A}_{k-1} \mathbf{U}_{k-1} \mathbf{A}_{k-1}^{T}+\mathbf{Q}_{k-1},
\end{aligned}
$$


where $\mathbf{m}_{k}^{-}$and $\mathbf{U}_{k}^{-}$are the predicted mean vector and the predicted covariance matrix of the state in sampling window $k$, respectively, while $\mathbf{m}_{k-1}$ and $\mathbf{U}_{k-1}$ are the estimated mean vector and the estimated covariance matrix of the state in sampling window $k-1$. Mean and covariance of states consist of position and velocity information of the target device.

\section{Update}

$$
\begin{aligned}
\mathbf{v}_{k} & =\mathbf{y}_{k}-\mathbf{H}_{k} \mathbf{m}_{k}^{-} \\
\mathbf{S}_{k} & =\mathbf{H}_{k} \mathbf{U}_{k}^{-} \mathbf{H}_{k}^{T}+\mathbf{R}_{k} \\
\mathbf{K}_{k} & =\mathbf{U}_{k}^{-} \mathbf{H}_{k}^{T} \mathbf{S}_{k}^{-1} \\
\mathbf{m}_{k} & =\mathbf{m}_{k}^{-}+\mathbf{K}_{k} \mathbf{v}_{k} \\
\mathbf{U}_{k} & =\mathbf{U}_{k}^{-}-\mathbf{K}_{k} \mathbf{S}_{k} \mathbf{K}_{k}^{T}
\end{aligned}
$$

where $\mathbf{v}_{k}$ and $\mathbf{S}_{k}$ are the measurement residual and measurement prediction covariance on state for sampling window $k$, respectively. Kalman gain $\mathbf{K}_{k}$ is the tuning parameter, which adjusts prediction's correction for sampling window $k$. Estimated mean and covariance of the state at sampling window $k$ with adding the measurements are represented with $\mathbf{m}_{\boldsymbol{k}}$ and $\mathbf{U}_{k}$, respectively.

\subsubsection{IMM Based Kalman Tracking of Target Location}

Section 4.4.1 summarized the model for a generic Kalman filter; in this section, we consider a multi-modal Kalman filter, whose parameters change with respect to the model order, and the models are selected based on the number of available RSS measurements at different reference nodes. These models also vary by their measurement error since the number of measurements affects the localization accuracy. Each filter 
receives a raw location estimate as an input (relying solely on measurements), and outputs a filtered location output using a technique that varies by the model.

First, consider that the RSS of the probe request from the target mobile device is available only at a single reference node (i.e. $\left|\mathcal{P}_{k}\right|=1$ ). Then, since we have access to prior location estimate, a better location estimate than that was introduced in 4.4 and Fig. 4.4(a) can be obtained, as summarized in Fig. 4.4(b) as Model-1 $(j=1)$. The target node is assumed to be on the straight line between the predicted position for sampling window $k$ (represented by $\boldsymbol{x}_{k}^{-}$), and the position of the available reference node $\left(\boldsymbol{x}_{i}\right)$, utilizing ranging information from the reference node as

$$
\hat{\boldsymbol{x}}_{k}^{\prime}=\boldsymbol{x}_{i}+\hat{d}_{i, k} \frac{\boldsymbol{x}_{k}^{--}-\boldsymbol{x}_{i}}{\left\|\boldsymbol{x}_{k}^{--}-\boldsymbol{x}_{i}\right\|}
$$

where $\hat{d}_{i, k}$ is as in 4.6). In other words, position of target node is estimated as $\hat{d}_{i, k}$ far from available reference node (from measurements), towards predicted position by IMM. In particular, the predicted position based on previous location estimate and trajectory are used for reducing the error of the estimation in Fig. 4.4(a) which relied only on a single reference node measurement.

When $\left|\mathcal{P}_{k}\right|=2$ (probe RSS measurement available at two reference nodes), a similar approach as illustrated in Fig. 4.4(d) called as Model-2 is followed. In particular, we consider the predicted position of the target node $\left(\boldsymbol{x}_{k}^{\prime-}\right)$ in 4.14 for compensating bias with estimated location in (4.5) as

$$
\hat{\boldsymbol{x}}_{k}^{\prime}=\frac{\boldsymbol{x}_{k}^{\prime-}+\boldsymbol{x}_{i}+\left(\hat{d}_{i, k}+\frac{\hat{d}_{i, k}+\hat{d}_{u, k}-d_{i u}}{2}\right) \frac{\boldsymbol{x}_{u}-\boldsymbol{x}_{i}}{\left\|\boldsymbol{x}_{u}-\boldsymbol{x}_{i}\right\|}}{2} .
$$

Simply, the position of the target node is first estimated as in 4.5 using the measurements from the two reference nodes, and then the bias of the estimation is adjusted with the predicted position by taking average of the estimate and the prediction.

Finally, in Model-3 (i.e. when $\left|\mathcal{P}_{k}\right| \geq 3$ ), LLS or NLS estimator is used based on the number of reference nodes and unknown parameters. If either $P_{0}$ or $n$ is 


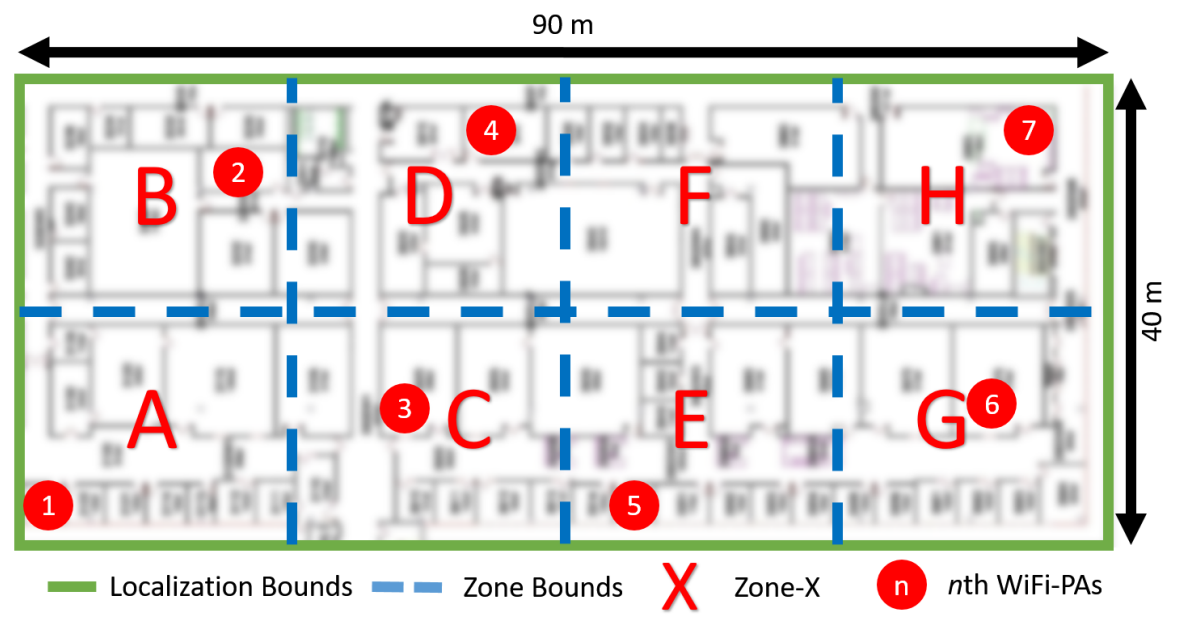

Figure 4.5: Map of building floor used in experiments with eight different building zones, as well as the locations of the WiFi sniffers placed across the different zones.

unavailable, we use NLS to estimate the location as well as that parameter, as described in Section 4.3.2. If there are no unknown parameters (i.e. all the parameters are estimated), LLS is used to estimate the location due to its lower computational complexity.

In each model, the $2 \times 2$ measurement noise covariance matrix $\mathbf{R}_{k}^{j}$ varies with the number of measurements as follows:

$$
\mathbf{R}_{k}^{j}= \begin{cases}\operatorname{diag}\left(\sigma_{M}^{2} / 1, \sigma_{M}^{2} / 1\right), & \text { if } j=1 \\ \operatorname{diag}\left(\sigma_{M}^{2} / 2, \sigma_{M}^{2} / 2\right), & \text { if } j=2, \\ \operatorname{diag}\left(\sigma_{M}^{2} / 4, \sigma_{M}^{2} / 4\right), & \text { if } j=3\end{cases}
$$

where $\sigma_{M}^{2}$ represents the variance of the error in the location estimates. Using the covariance matrix as in 4.23 for different models ensures that the prediction is weighted more when there are limited number of available reference nodes, while measurements are weighted more when there are at least three or more available reference nodes. 


\subsubsection{Occupancy Counting}

Our final goal is real-time occupancy monitoring, by counting the number of users within coarsely defined occupancy zones in the area of interest. The IMM filtering results lead to mapping of the each target node to a zone which is created as illustrated in Fig. 4.1. Sum of the individual target nodes in a zone gives the occupancy count of that zone. In this case, there might be a problem when a user switches the device's screen off since probe requests may not be sent for 300 seconds to 600 seconds 93 . Thus, this interval should be considered as a grace period for that individual device, and the user should be kept within the occupancy count for that zone, until the time threshold expires. In our experimental model, we consider the grace period to be 300 seconds, after which we remove a user from the building zone which has been last localized.

\subsection{Sniffing Probe Requests}

In the experiments, seven different WiFi-PA equipment are deployed at various locations on the floor of a university campus building as in Fig. 4.5. The data captured at WiFi-PA include absolute time stamp providing the time at which the data was captured, MAC address of the WiFi device, and the RSS of the WiFi device. Gathered data is transferred to a server for execution of the occupancy counting.

Probe request mechanism is explained in $[76]$. Probe requests, which are sent by WiFi devices to scan WiFi APs at certain channels, are active mechanisms to discover APs around the mobile device. APs respond with probe responses and beacons. A problem with probe requests is passing-by and static devices. For instance, there might be people outside of the building passing by a WiFi device and it still can be detected by the reference nodes which are close to the border. Such devices usually 


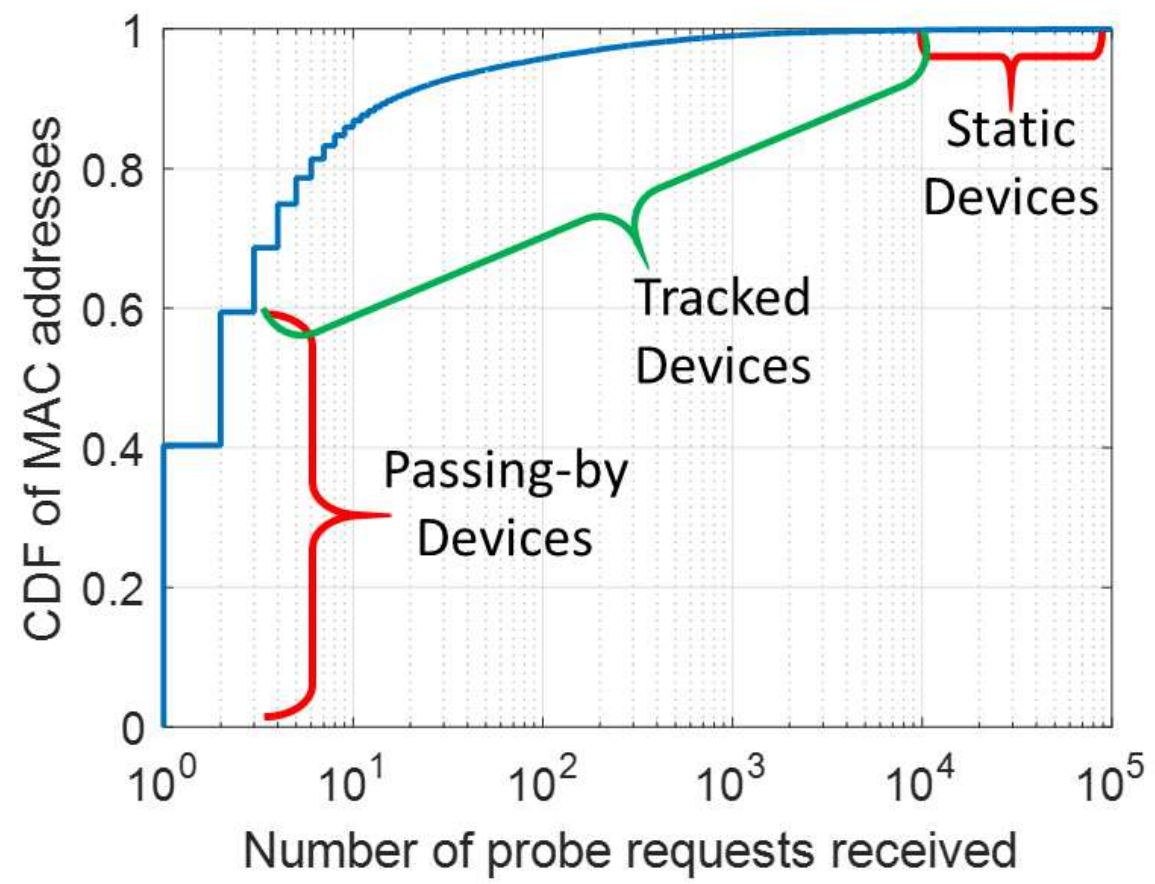

Figure 4.6: Distribution of passing-by, static and tracked devices by number of probe requests.

send a few low power probe requests while in the vicinity of the reference node. Static devices (such as wireless printers) send thousands of probe requests with the same RSS value usually periodically. Passing-by and static devices are removed from the occupancy count in our analysis using outlier detection and filtering techniques. Our experimental results indicate that only $40 \%$ of the detected devices are in our region of interest as shown in Fig. 4.6. In total, 33, 847 unique and legitimate MAC addresses are detected within a period of one week.

Since probe requests are only used to discover APs, they are burst and intermittent as shown in Fig. 4.3. After connecting to a network, probe requests are rarely sent to search for a network with better quality or handover possibilities. Therefore, they could be sent 50 times within a minute (i.e., burst), or do not trigger for 5 to 10 minutes (i.e., intermittent). This depends on the implementation of the IEEE 802.11 
Table 4.2: Simulation parameters of WiFi Probe Request-based tracking.

\begin{tabular}{|l|l|}
\hline Parameter & Value \\
\hline \hline WiFi protocol & IEEE $802.11 \mathrm{n}$ \\
\hline Floor width and length & $40 \mathrm{~m} \times 90 \mathrm{~m}$ \\
\hline Sampling window length $(\mathrm{T})$ & 3 seconds \\
\hline Sampling window hold length $(\mathrm{L})$ & 1 to 15 \\
\hline Occupancy grace period & 5 minutes \\
\hline Number of the reference nodes & 7 \\
\hline Path loss exponent $(n)$ & $2-6$ \\
\hline Reference distance $\left(d_{0}\right)$ & 1 meter \\
\hline Signal strength at reference distance $\left(P_{0}\right)$ & $-35 \mathrm{dBm}$ \\
\hline Noise floor & $-90 \mathrm{dBm}$ \\
\hline Detection threshold & $-90 \mathrm{dBm}$ \\
\hline
\end{tabular}

protocol at the device in terms of both the design of hardware and software. Due to the mobility, WiFi device needs to send probe requests more frequently since it causes to lose connectivity with the AP, or degrade the quality of the connection. Therefore, probe requests can effectively facilitate localization and tracking of the WiFi devices.

\subsection{Numerical Results}

In this section, we will first investigate the performance of the proposed framework with computer simulations using realistic WiFi signal propagation data. Subsequently, we will present our experimental results and occupancy counting based on the measurement data that has been collected at FIU College of Engineering and Computing building.

\subsubsection{Simulation Results}

The proposed framework is simulated considering an indoor environment with propagation parameters and indoor channel characteristics summarized in Table 4.2. In our 


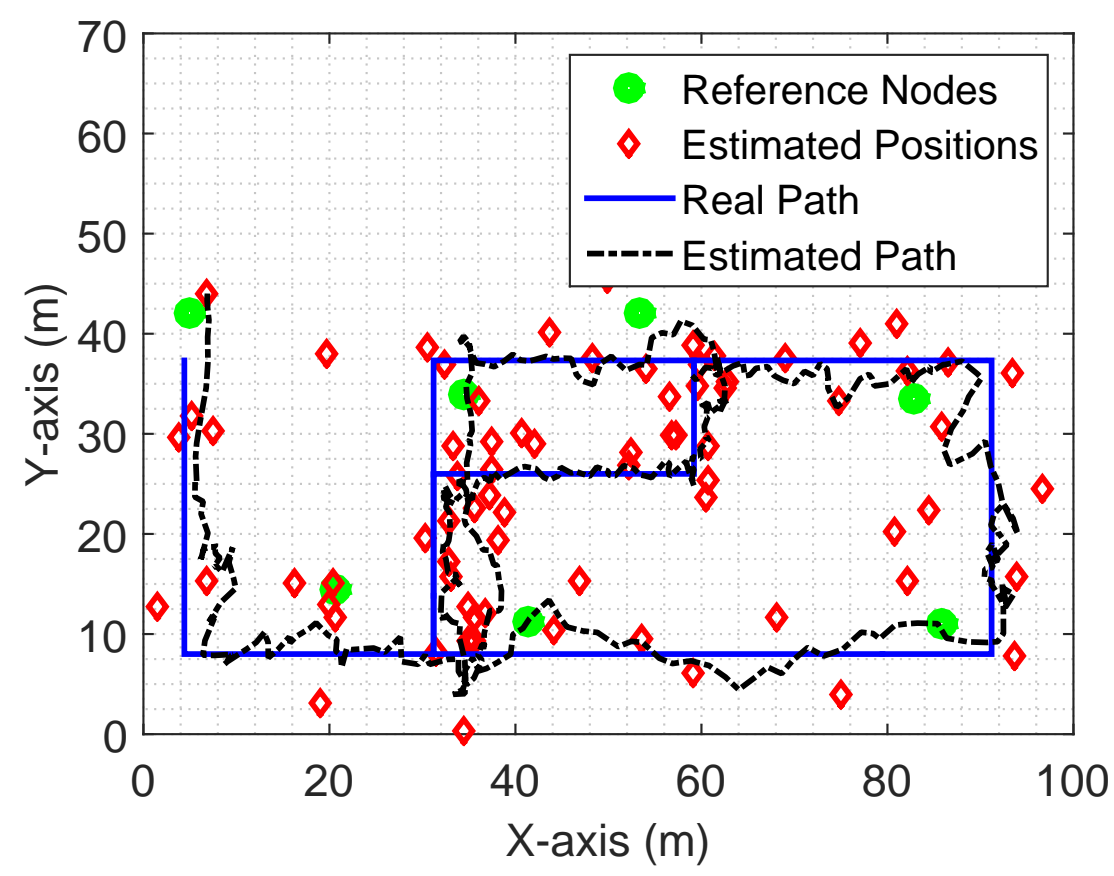

Figure 4.7: A sample simulation for a given map (Fig. 4.5).

simulations, we used a realistic multipath indoor channel model to generate the RSS values. It is compared with the ideal flat fading channel results, where there is only a single strong path together with the AWGN. Probe request statistics such as interarrival times and detection probability are included from experimental measurements as well to increase the reliability of the results. By introducing detection probability, detection of probe requests by single or multiple reference nodes is modeled realistic. Also, path loss exponents and reference received powers are used from the obtained measurements.

An example trajectory from the simulations are plotted as seen in Fig. 4.7 with the individual position estimates before filtering, as well as the IMM output as the estimated path. The estimated path is very close to the real path as we expect. The mean RMSE for the estimated path is 3.83 meters in this realization, while mean RMSE for individual localization estimates is 6.17 meters which is significantly higher. Cumulative distribution function (CDF) of the mean tracking RMSE for bare 


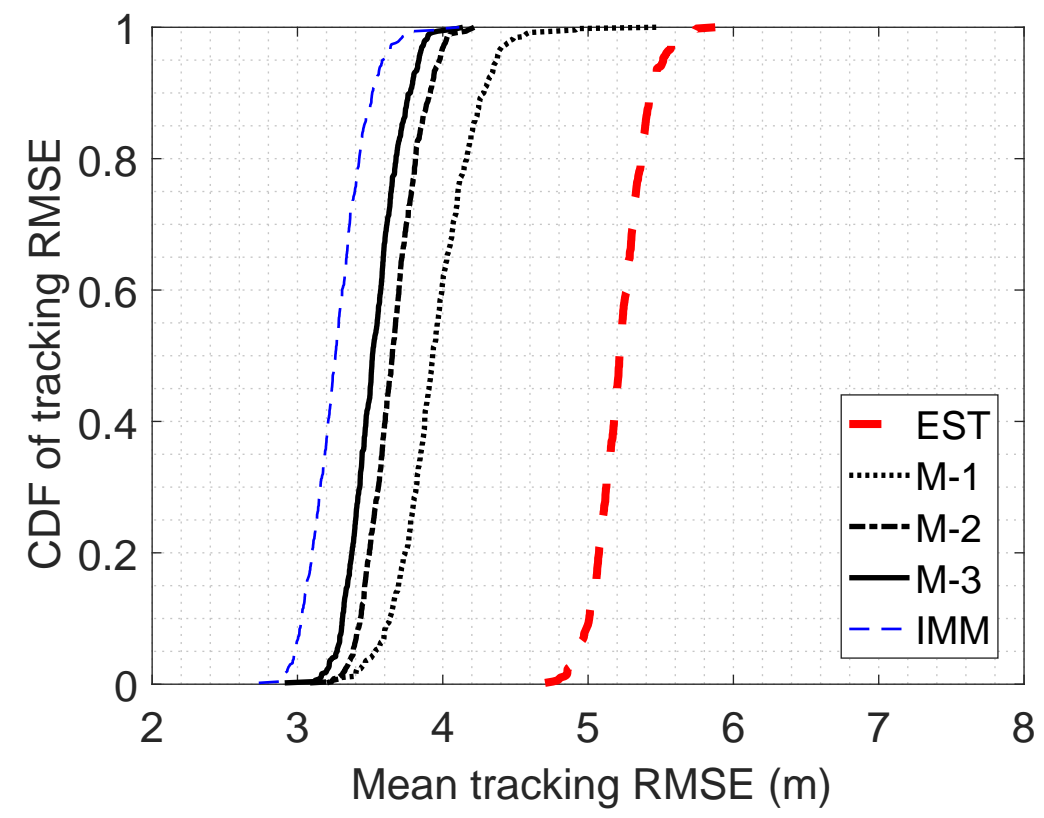

Figure 4.8: CDF of mean tracking RMSE for the simulation.

localization estimates without IMM filtering (EST), and the IMM filtering results are compared for a given scenario in Fig. 4.8. Simulations are averaged over 1000 Monte Carlo realizations with the realistic indoor channel parameters. Median RMSE for IMM filter is 3.59 meters, whereas 4.01, 3.72, and 4.13 meters for Model-1 (M1), Model-2 (M-2), and Model-3 (M-3), respectively. It is also 5.97 meters for the individual location estimates (EST), which is almost twice the RMSE obtained from the IMM filter.

This performance is also reflected to the zone-level localization performance (i.e., percentage of correctly localizing a target device within the building zone where its true location belongs) as shown in Fig. 4.9. Even though the zone-level localization performance varies from $88 \%$ to $97 \%$ for IMM, the median performance is $92.5 \%$. Individual Kalman filters are very close to each other with the median performance of $88.5 \%$ with range of $80 \%$ to $95 \%$. Similar to the tracking RMSE, performance of 


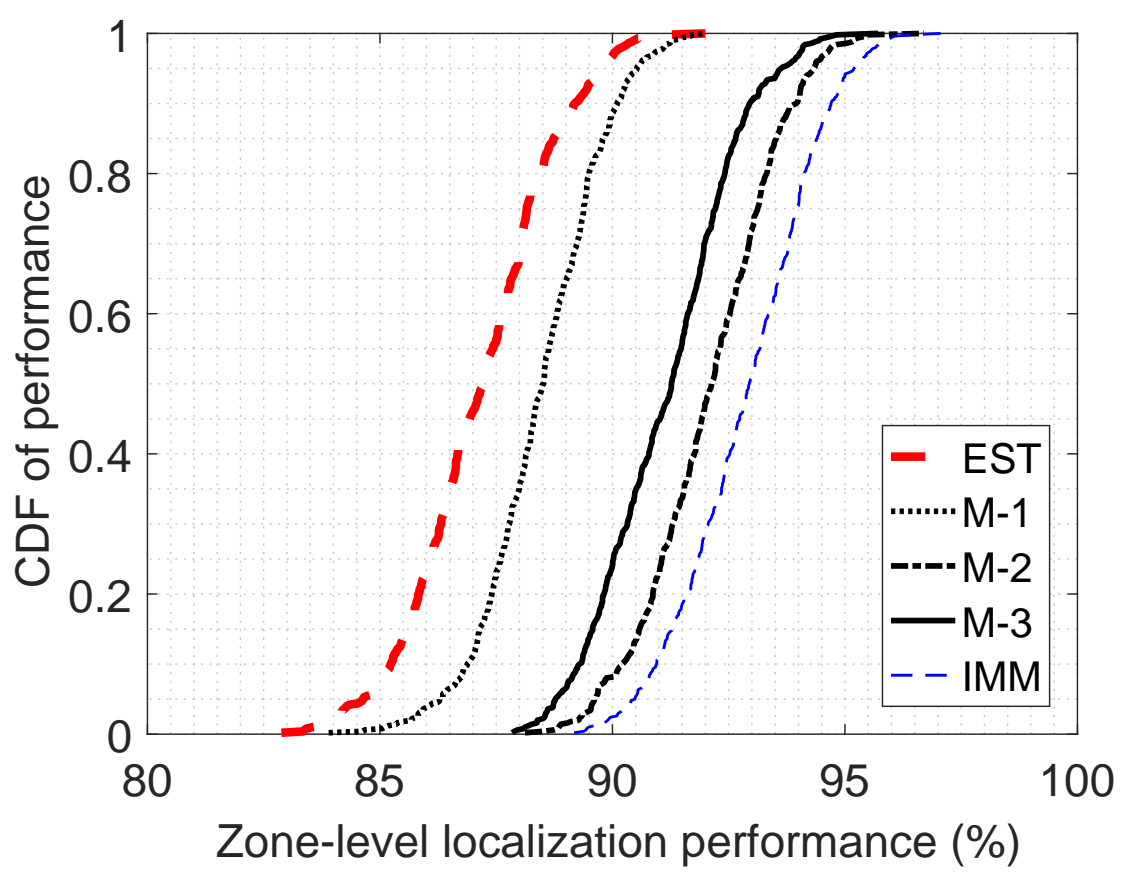

Figure 4.9: Zone-level localization performance.

zone-level localization is much lower for individual location estimates as the median performance is $83.2 \%$, with a range between $76 \%$ to $87 \%$.

\subsubsection{Experimental Results}

In addition to simulations, extensive experimental data are collected at FIU to validate the proposed methods with real measurements. First, several experimental walks are considered using WiFi devices to compare the simulation and experimental results of the ground-truth data on a fixed path within the building, using the reference nodes as shown in Fig. 4.7. The track is walked at different times of the day along with several WiFi devices. The comparison of the zone-level localization performance is presented in Fig. 4.10, The performance of simulations with the AWGN and realistic indoor channel are close to each other for both the individual location estimates and the IMM. The median performance of the individual location estimates is $84 \%$ under 


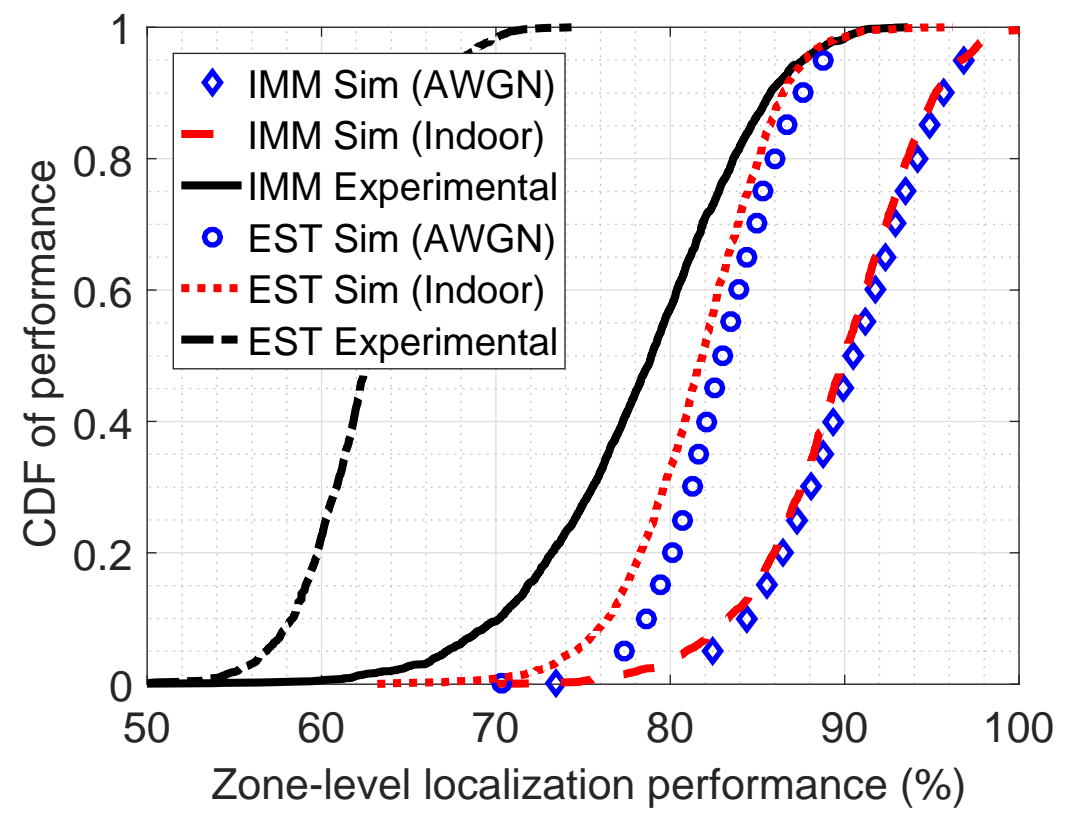

Figure 4.10: Simulation and experiment performance comparison of zone-level localization with and without IMM.

ideal AWGN, whereas it is $83 \%$ under realistic indoor channel. Performance with ground-truth data is lower with $64 \%$. The IMM improves the median performance to $90 \%$ in the simulation results, whereas it increases to $78 \%$ in the experimental results with real data. The growth in the experimental performance using IMM is substantial with $14 \%$ increase. Hence, the IMM plays a critical role in the zone-level localization of the individual target devices.

Once we localize all the target mobile users in building zones (filtered out from passing by users and other fixed devices as shown in Fig. 4.6) using IMM, we can obtain the real-time occupancy count in different building zones using these location estimates. Using experimental data, occupancy counting results over a period of 24 hours are shown in Fig. 4.11 as a sum of total occupants over all the zones in a week day. As expected, peak hours of the occupancy in the building is between $12 \mathrm{PM}$ and 6 PM, where most of the classes are scheduled. The lowest occupancy is around 5 AM. Fig. 4.11 also shows that if we enforce the localization and occupancy counting 


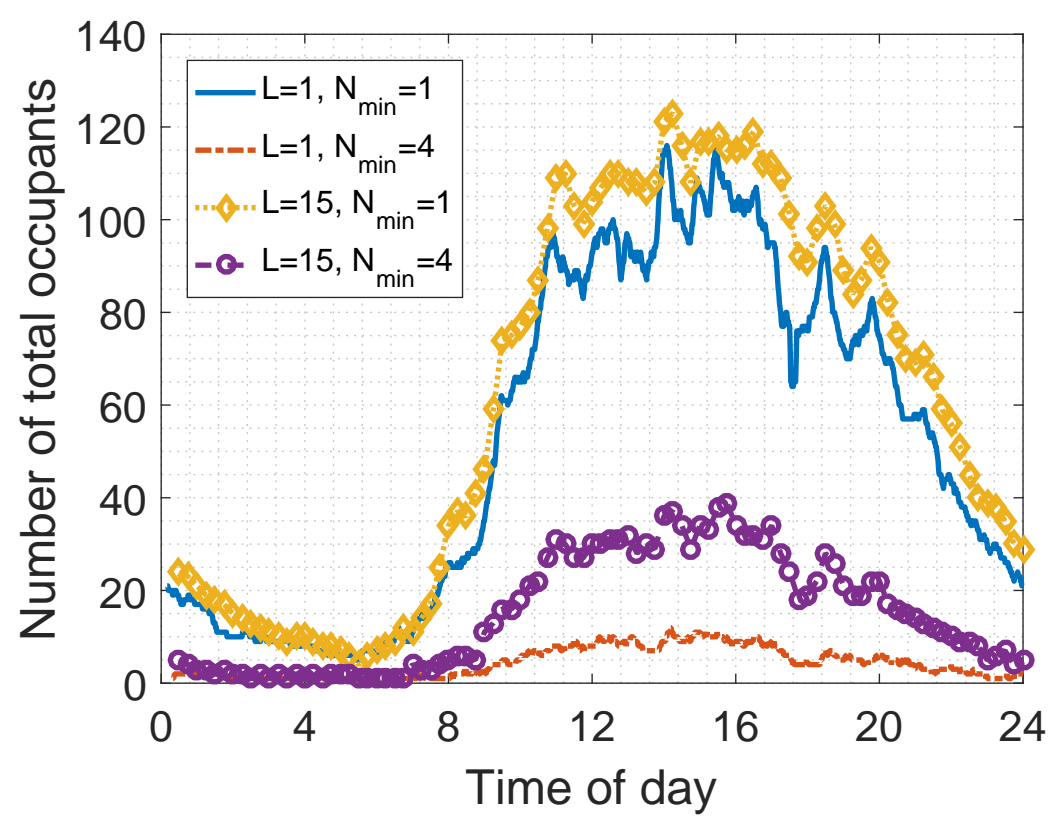

Figure 4.11: Total number of occupants on a weekday for sample-and-hold window lengths $L=1$ and $L=15$. Scenario with $N_{\min }=4$ necessitates at least four reference nodes to localize a target device, while $N_{\min }=1$ can localize a target device within a building zone using a single reference node.

technique to use at least $N_{\min }=4$ reference nodes $\left(\left|\mathcal{P}_{k}\right| \geq 4\right)$, a significant portion of the occupancy is not detected. Therefore, the proposed techniques in Fig. 4.4 and the IMM method can help in significantly improving the occupancy counting.

Fig. 4.11 also compares the effects of the sample-and-hold window length $L$ on occupancy counting results. Even though the total occupancy count with $N_{\min }=4$ (i.e. the measurements are dropped if $\left|\mathcal{P}_{k}\right| \leq 3 \mid$ ) at peak hours increases to 40 using a sample-and-hold method using holding length of $L=15$, this is still significantly lower compared to when we use IMM based tracking with $N_{\min }=1$ (i.e. measurements are used regardless of number of available reference nodes). Use of a larger holding window also smooths the total occupancy count when the IMM approach is used.

In Fig. 4.12 and Fig. 4.13 , we provide occupancy counting results in each of the eight individual zones over a period of one whole week day and four hours, respectively, considering different time resolutions. Although the individual zones show similar 


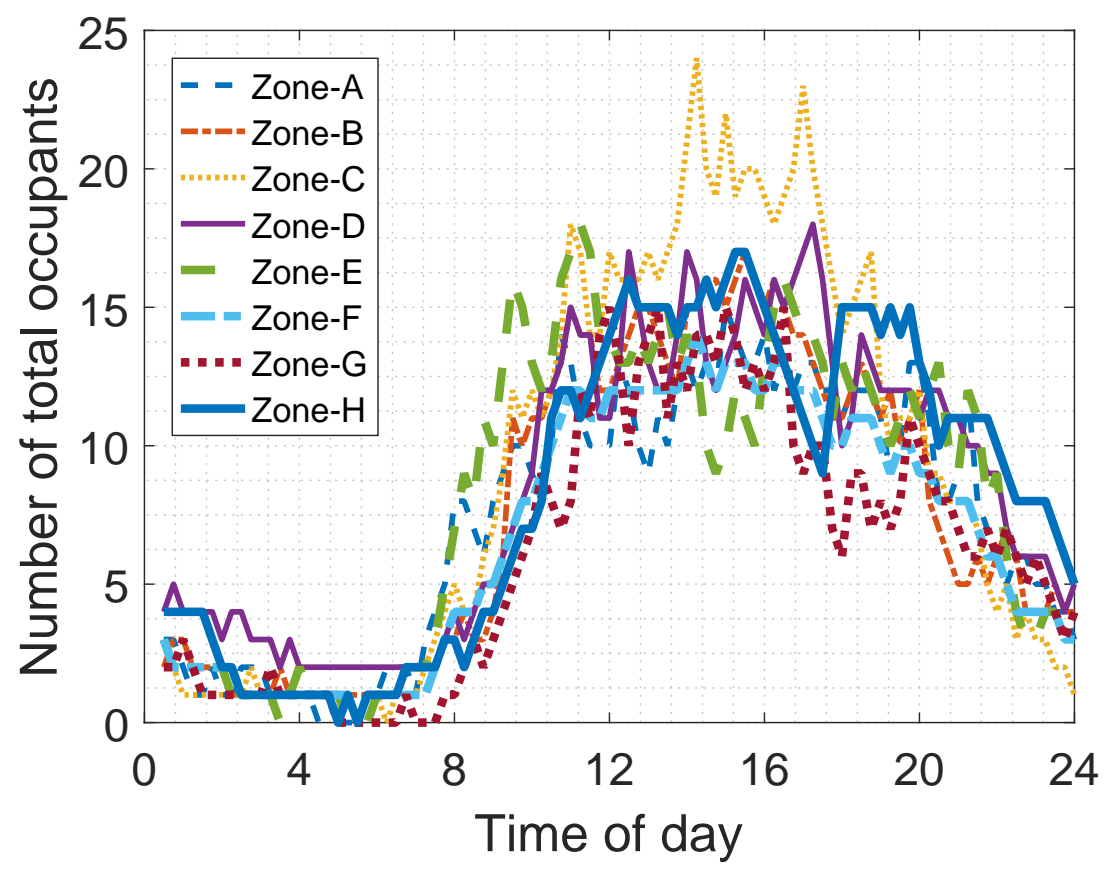

Figure 4.12: Total number of occupants within the individual zones given in Fig. 4.5 throughout a weekday.

behavior throughout the day, their peak hours are different as seen in Fig. 4.12. For instance, Zone-E has a peak at 11 AM, while Zone-D has its peak at 5 PM. The most common characteristic is that all of the zones have their minimum around $5 \mathrm{AM}$ in the morning. Zone-C has the highest peak among all zones, with 24 people around 3 PM. Another interesting observation is that Zone-H, which consists a student study area and senior design laboratory, has occupants until very late hours, while Zone-C has least occupied zone at midnight, since it consists mostly administrative offices. In Fig. 4.13, occupancy counts of individual zones are shown within peak hours, i.e. from $10 \mathrm{AM}$ to $2 \mathrm{PM}$. The movement of people from one zone to another is more visible in this figure.

In Fig. 4.14, percentage of occupants based on hours spent in the building is presented. For instance, $11.5 \%$ of the people spend 2 to 3 hours in the building, whereas the people spending less than 1 hour is less than $1 \%$. More than half of the 


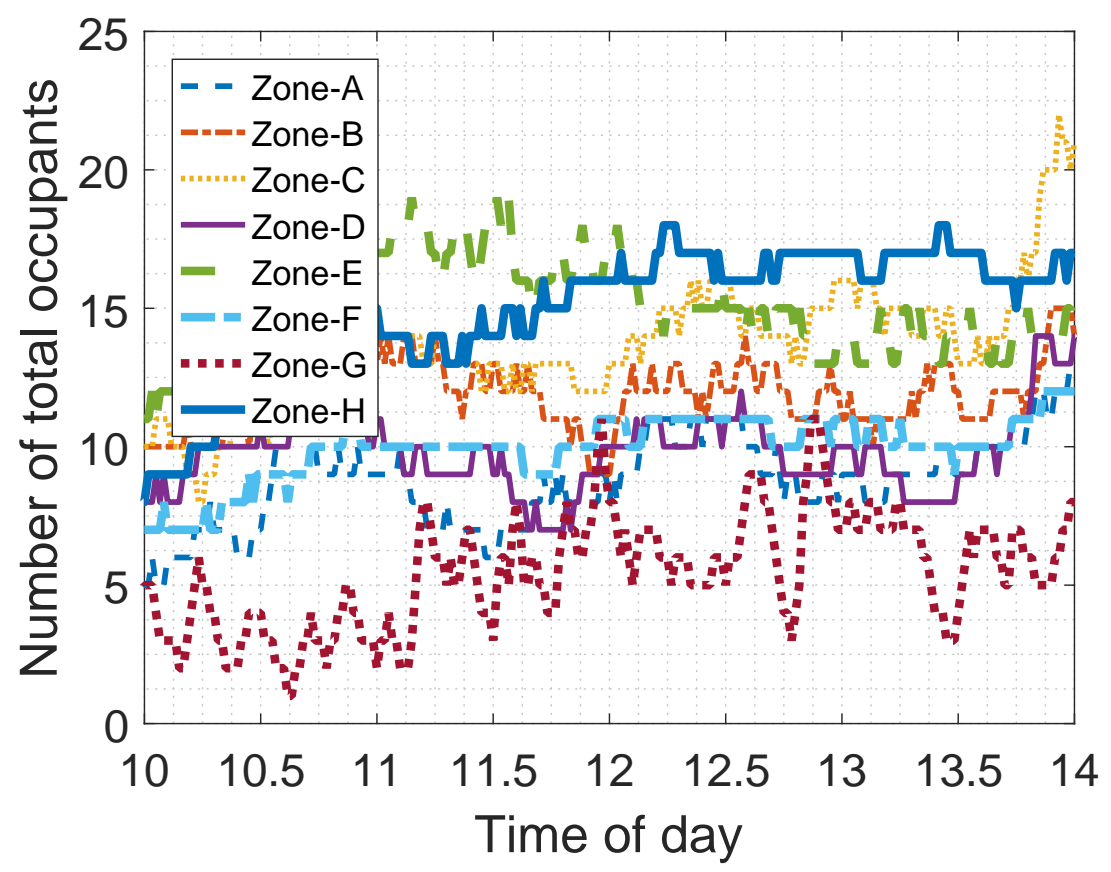

Figure 4.13: Total number of occupants within individual zones given in Fig. 4.5 at peak hours.

occupants spend less than 6 hours in the building, with $13 \%$ of them spending 3 to 4 hours.

\subsection{Conclusion}

In this chapter, we proposed a new framework for real-time occupancy counting of buildings by passively sniffing WiFi probe requests. We first differentiate the static and passing-by devices in the building using probe request statistics, and then use the information for the remaining users for localization, tracking, and occupancy counting inside the building. The proposed method assures the utilization of the probe requests regardless of the number of the available reference nodes that they are detected, which allows to detect three times more users in the occupancy counting process when compared to requiring at at least four reference nodes for localization. 


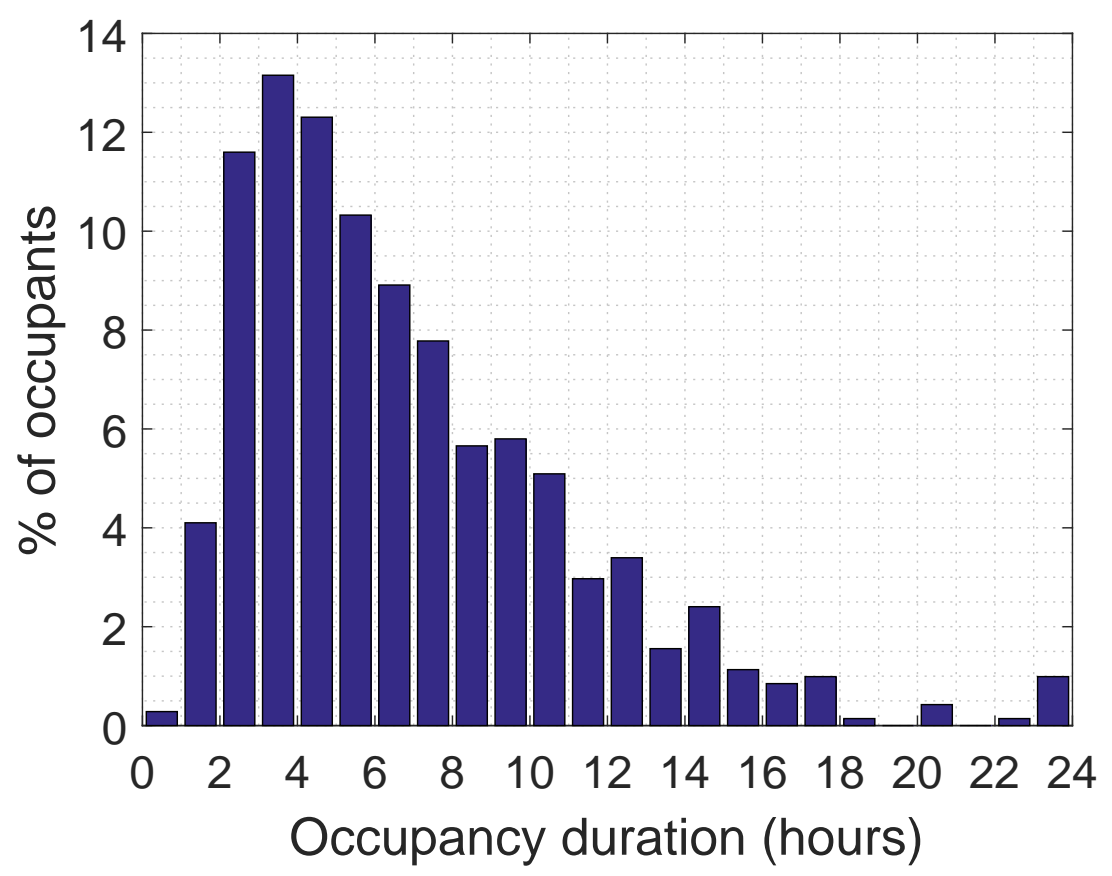

Figure 4.14: The distribution of the occupancy hours for the individual occupants.

In our experimental results, we are able to perform zone-level occupancy tracking with up to $90 \%$ accuracy. We are also able to determine the peak hours of the individual zones as well as the quiet times of the building. Our future work includes use of more advanced filtering techniques such as particle filters to further improve zone level localization and occupancy counting performance. 


\section{CHAPTER 5}

\section{INDOOR UAV NAVIGATION}

Search and rescue, public safety, and emergency management applications may require navigation of first responders to a victim's location. This can be achieved by using the signals radiated from Internet of Things (IoT) devices carried by the victims such as mobile phones, smart watches, fitness trackers, or other smart sensors, which can be probed to send RF signals repetitively [94,95]. To this end, unmanned aerial vehicles (UAVs) have been recently gaining more attention due to communication, autonomous navigation, and video capture capabilities, and they can help in localizing people during emergency situations. For example, they can be quickly deployed within a building on fire to localize victims and first responders, to deliver first aid kits, and to maintain wireless connectivity with them for enabling real-time situational awareness through live video.

In this chapter, as shown in Fig. 5.1, we consider the problem of navigating a UAV to a Rayleigh fading RF source. We consider a GPS-denied indoor environment and assume that the RF source continuously radiates signals. For example, most mobile equipment continuously transmit $\mathrm{WiFi}$ probe requests to discover nearby access points [96], and a mobile device may also be forced by a UAV to transmit wireless signals in case of emergency incidents. In the literature, collaborative localization of a moving RF source is presented in [97] by a swarm of UAVs based on the D-optimality criteria. Another study in [98 presents an optimal flying path for UAV-assisted IoT sensor networks using a location aware multi-layer information map. It considers different utility functions based on the sensor density, energy consumption, flight time, and flying risk level, and weighted sum of multi-objective utility functions is maximized using a genetic algorithm. Several other works in the literature

consider Q-learning and other reinforcement learning (RL) techniques for naviga- 
tion of robots 99,100 . To our best knowledge, there are no studies that consider the use of Q-learning for navigation of UAVs based on the received signal strength (RSS) observations from a Rayleigh fading RF source. In particular, in indoor environments, Rayleigh fading signal from the source may cause significant variations in the RSS model, and hence it can bias the navigation algorithms while deciding on the optimum actions for the UAV. On the other hand, Q-learning is a model-free reinforcement learning technique which avoids bias in the navigation of UAV.

In this chapter, we study the behavior of Q-learning based UAV navigation under Rayleigh fading assumption, and investigate averaging of the RSS over different time spans considering different UAV speeds. We also study a variable learning rate technique, which is shown to provide better convergence time in reaching to the Rayleigh fading source when compared with a fixed learning rate technique. We compare the proposed algorithm with an existing Reinforcement Learning (RL) technique [101]. Contributions of this work can be summarized as follows:

- A model-free Q-learning algorithm for indoor UAV navigation to a Rayleigh fading RF source is proposed

- Proposed algorithm is compared with an existing RL based technique

- Varying and fixed learning rates are studied for convergence time

- Various UAV speeds are studied for convergence time

System Model: The RSS at a UAV from a Rayleigh fading wireless source can be calculated using the distance between the source and the UAV as $P_{\mathrm{R}}=P_{\mathrm{Tx}}-P L(d)-$ $S$, where $P_{\mathrm{R}}$ is the RSS, $P_{\mathrm{Tx}}$ is the transmit power of the source, $P L(d)$ is the path loss at distance $d$, and $S$ is a random variable that captures Rayleigh fading which may cause deep fades in the RSS. In this letter, we consider 3GPP TR 36.814 path loss model [102], given by $P L(d)=128.1+37.6 \log _{10}(d / 1000)$. 


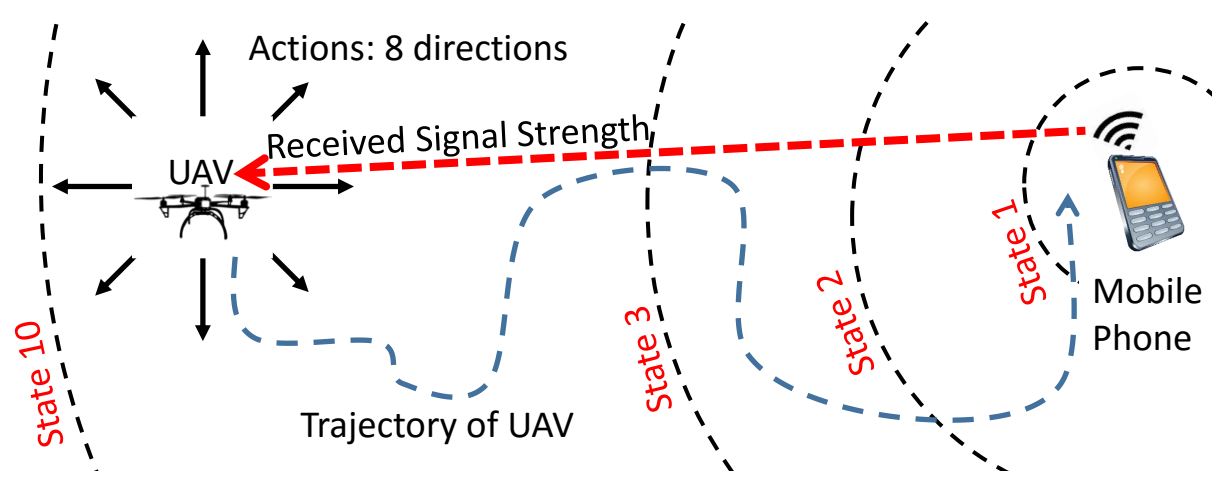

Figure 5.1: Navigation to a Rayleigh fading wireless source.

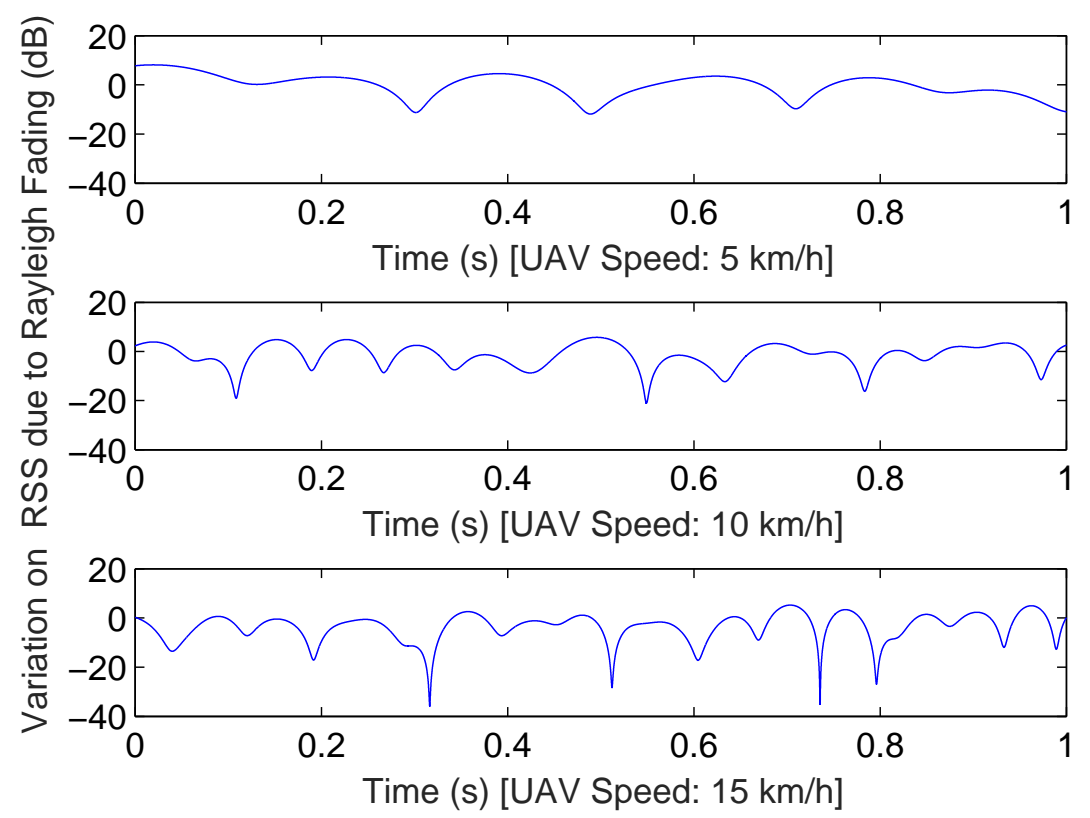

Figure 5.2: Rayleigh fading RSS for three UAV velocities.

As an example, in Fig. 5.2, we present Rayleigh fading RSS for three different UAV velocities, considering a fixed RF source. Results show that the RSS may observe deep fades which may cause as large as $40 \mathrm{~dB}$ losses for certain cases. Moreover, the variation of the RSS increases with larger UAV speeds as shown in Fig. 5.2. Such deep fades may bias the UAV that it may be navigating in the wrong direction, even when the UAV may be approaching closer to the target node. In the next section, we will present the proposed Q-learning algorithm, and discuss how the Rayleigh fading effects as in Fig. 5.2 can be mitigated for navigation to target. 


\begin{tabular}{|c|c|}
\hline State & RSS $(\mathbf{d B m})$ \\
\hline$s=1$ & $P_{\mathrm{R}}>-40$ \\
\hline$s=2$ & $-50 \leq P_{\mathrm{R}} \leq-40$ \\
\hline$s=3$ & $-60 \leq P_{\mathrm{R}} \leq-50$ \\
\hline$s=4$ & $-70 \leq P_{\mathrm{R}} \leq-60$ \\
\hline$s=5$ & $-80 \leq P_{\mathrm{R}} \leq-70$ \\
\hline$s=6$ & $-90 \leq P_{\mathrm{R}} \leq-80$ \\
\hline$s=7$ & $-100 \leq P_{\mathrm{R}} \leq-90$ \\
\hline$s=8$ & $-110 \leq P_{\mathrm{R}} \leq-100$ \\
\hline$s=9$ & $-120 \leq P_{\mathrm{R}} \leq-110$ \\
\hline$s=10$ & $P_{\mathrm{R}}<-120$ \\
\hline
\end{tabular}

Table 5.1: UAV states with respect to source RSS.

\subsection{Q-Learning Based Source Tracking}

We consider the use of Q-learning [103, 104] algorithm to navigate the UAV towards the wireless source based on the RSS observations at the UAV. Q-learning is an improved RL technique which can operate without any prior knowledge about the environment or the model for RSS observations. It learns by trial and error, and iteratively builds a value function of each state-action pair. The goal is to select the action which has maximum $Q$-value using following update rule at each step:

$$
Q(s, a) \leftarrow Q(s, a)+\alpha\left[r(s, a)+\gamma \max _{a^{\prime}} Q\left(s^{\prime}, a^{\prime}\right)-Q(s, a)\right]
$$

where $s^{\prime}$ is the state reached from state $s$ after action $a, \alpha \in[0,1]$ is the learning rate to control learning speed, and $r(s, a)$ is the immediate reward received as result of action $a$. We use two different learning rate models: a fixed learning rate with $\alpha$ set to a constant, 0.5 , and a varying learning rate where $\alpha$ is dynamically modified based on the observations. In varying learning rate model, as the quality of signal gets better (i.e. UAV is closer to source) learning rate increases not to miss or pass by the source. The discount factor is represented with $\gamma \in[0,1]$, which determines the importance of future rewards.

Balancing of exploration and exploitation is a critical issue in RL techniques 103 and there are several strategies to maintain this balance, such as $\epsilon$-greedy and softmax 


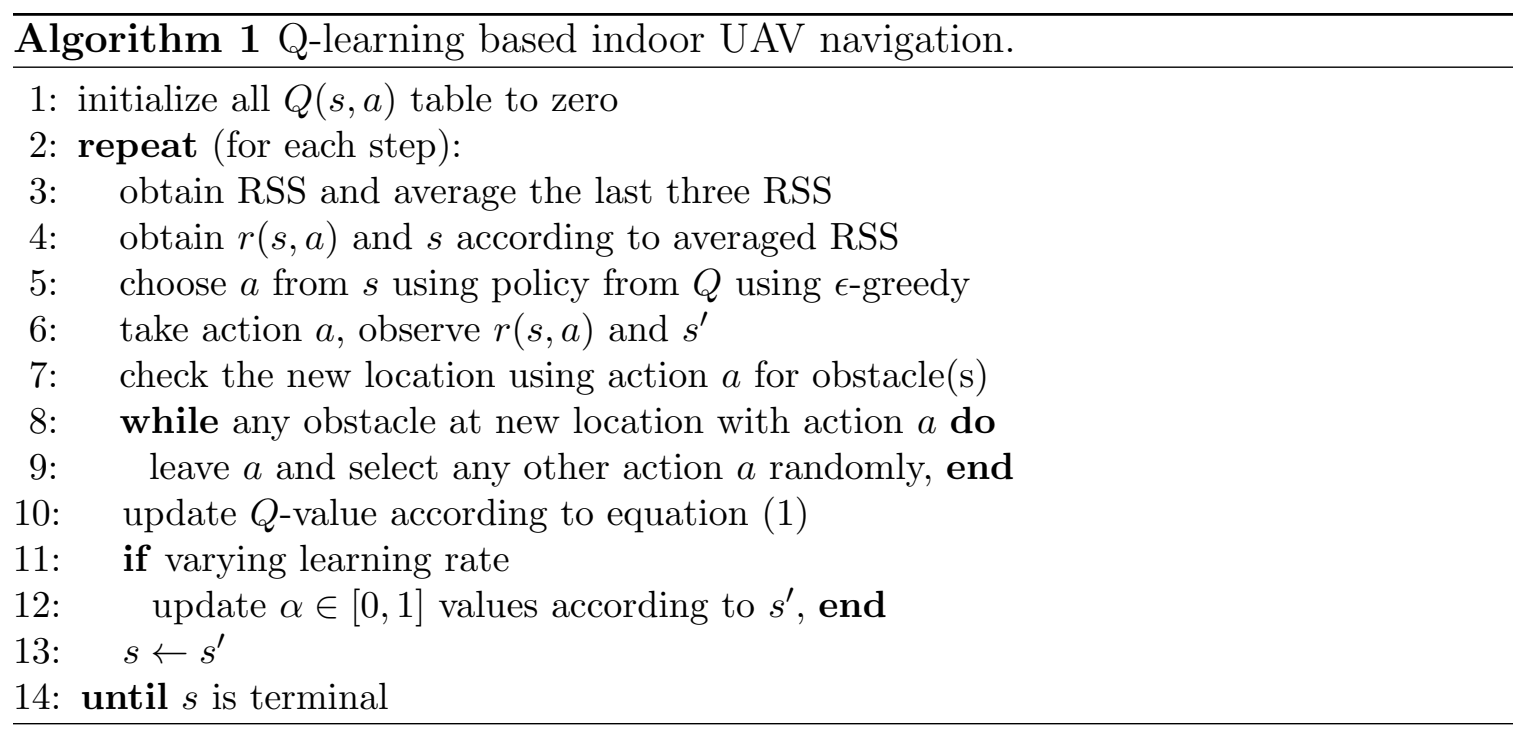

approaches 105. In here, the $\epsilon$-greedy exploration strategy is used, where $\epsilon \in[0,1]$ is the exploration probability. While the action is generally selected at each step according to highest Q-value for exploitation, for exploration the selection is carried out randomly with a small probability $\epsilon$. We consider that the RSS observed at the UAV belongs to a finite set $s \in\{1, \ldots, 10\}$ of 10 different states as in Table 5.1 .

The pseudo-code of the Q-learning technique that we used is presented in Algorithm 1. We consider two dimensional mobility and eight actions $a \in\{1, \ldots, 8\}$ for a UAV corresponding to eight uniformly-spaced directions in angular domain as shown in Fig. 5.1. This is because we assume that the UAV flies at a fixed height, and that the distance between the floor and the ceiling are insignificant compared to the distance between the UAV and the RF source. The Q-values of each state-action pair are then stored in a $10 \times 8$ matrix, initialized to zero at the beginning of the algorithm, and populated with the value of each specific action as new observations are obtained. The reward is set as the difference between the latest two values of the RSS, which increases the likelihood of choosing actions that will move the UAV towards the target. On the other hand, due to Rayleigh fading, UAV may also occasionally choose erroneous actions. 


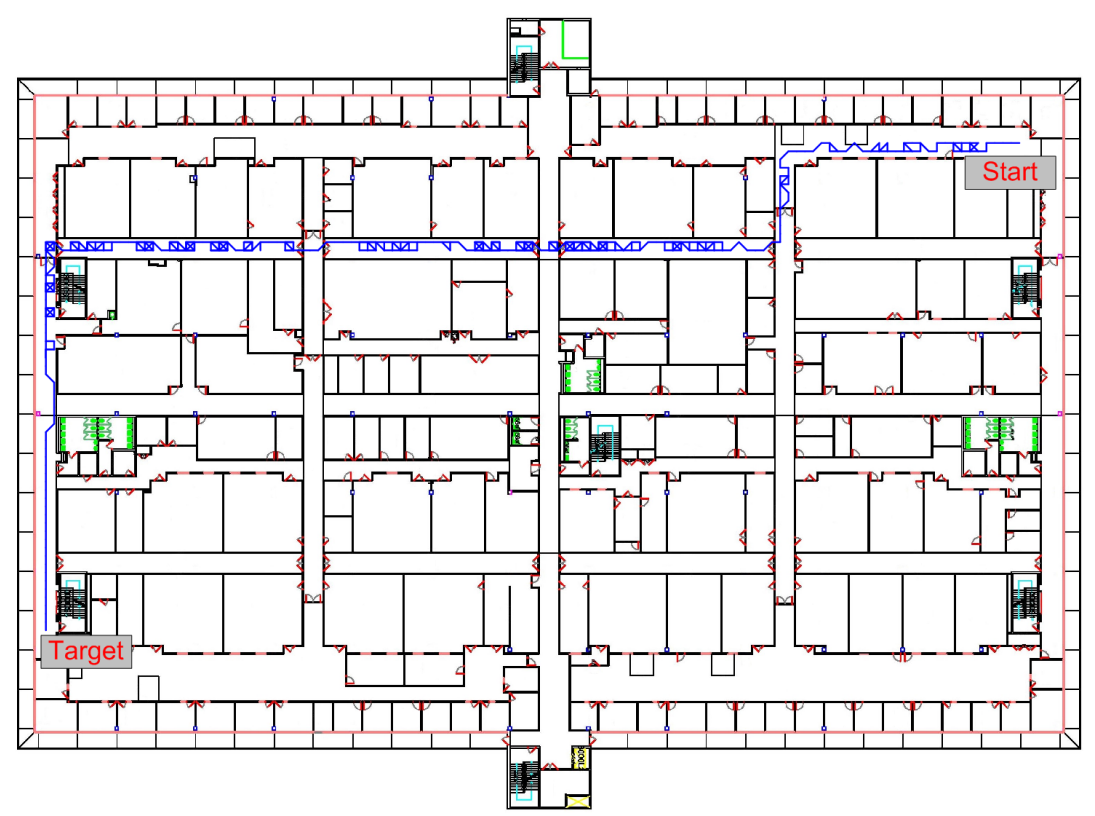

Figure 5.3: Map of FIU Engineering Center 3rd floor.

In order to minimize erroneous decisions due to deep fades in the RSS (see Fig. 5.2, we average the RSS over a sampling duration $T_{\mathrm{S}}$ before feeding it into the Q-learning algorithm. While a large averaging window $T_{\mathrm{S}}$ will help in minimizing wrong decisions due to deep fades, it will also introduce delays in choosing a new action value, and hence may delay the convergence time. On the other hand, a shorter averaging window will enable more frequent actions, albeit with more likelihood of RSS being subject to deep fades. Therefore, there is an optimum averaging window duration, which will result in the fastest navigation of the UAV to the RF source.

\subsection{Simulation Results and Conclusion}

The map of the FIU EC 3rd Floor (75 m by $120 \mathrm{~m}$ ) is used for simulations as shown in Fig. 5.3. It is assumed at the beginning of the simulation that the UAV and the RF source are positioned at the opposite corners of the floor considering a worst case scenario. The UAV can only navigate through the aisles on the map, without crashing 


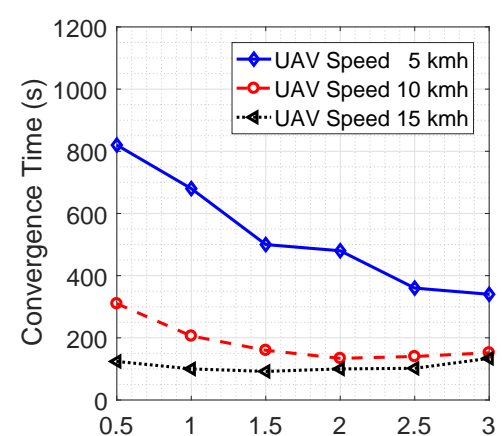

(a) Sampling Duration (s)

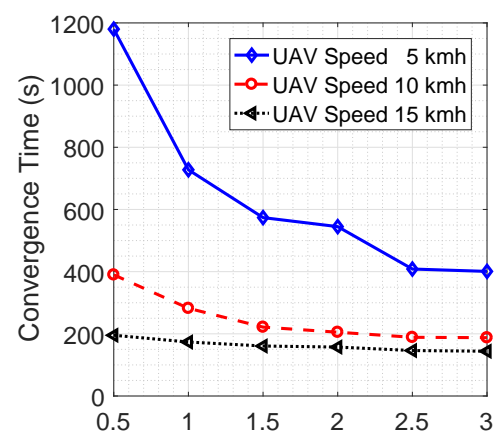

(b) Sampling Duration (s)

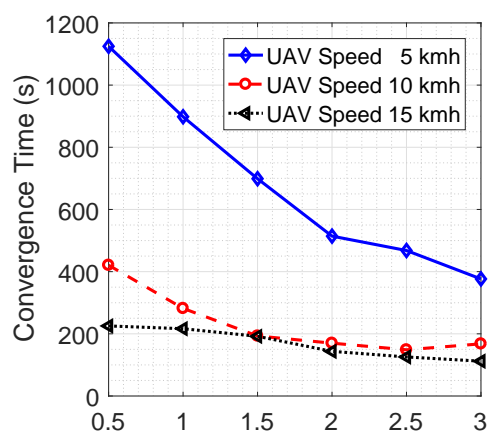

(c) Sampling Duration (s)

Figure 5.4: Convergence time of (a) Q-learning (varying learning rate); (b) Q-learning (fixed learning rate); (c) RL (single state).

into any walls. If any possible crash is detected for a selected action (e.g., through sonar sensors), the UAV tries a different action. The RSS is assumed to vary based on Rayleigh fading caused by the velocity of UAV and path loss dictated by the distance between the UAV and the target node.

Simulation results are obtained under several sampling durations, a variable (heuristically optimized) learning rate $\alpha$, and three different UAV speeds, as shown in Fig. 5.4. In Fig. 5.4(a) shows the convergence time for Q-learning with variable learning rate. The time it takes for the UAV to reach the vicinity of the target (i.e., the convergence time) tends to decrease with larger sampling duration for lower speeds. On the other hand, for high velocities, the convergence time starts increasing for larger sampling duration, since longer distances will be traveled by the UAV in case of wrong decisions. In general, larger velocity, at least for the considered set of three UAV velocities in this letter, results in faster convergence to the target node.

Use of fixed learning rate has a noticeable impact on the convergence performance of the Q-learning algorithm. In particular, Fig. 5.4(b) shows results with same UAV and target locations, and we present the convergence performance when the learning rate is fixed to 1 . Using a fixed learning rate decreases responsiveness of the system. Hence, the convergence time increases significantly. 
Finally, the proposed Q-learning algorithm is compared with the RL-based technique in 101]. In Fig. 5.4(c), the results for the RL-based technique is given. Although it achieves a similar performance to our proposed algorithm at higher velocities, our proposed algorithm performs better at lower velocities. Since the emergency situations may require delicate actions to avoid dangerous situations for a victim or first responder, lower velocities may be preferred in most cases.

In general, our overall results prove that it is critical to use a Q-learning based approach for avoiding the navigation bias in a Rayleigh fading environment with sufficiently large window. Another observation is that a variable learning rate is preferable compared to a fixed learning rate for increasing responsiveness of the system. 


\section{CHAPTER 6 \\ CONCLUDING REMARKS \& FUTURE WORK}

Wireless positioning and tracking systems for Internet of Things devices are expected to fulfill requirements of smart buildings and smart cities. There are many challenges associated with positioning and tracking systems. In this research we have studies some of the important challenges of which can be categorized into positioning with directional RF transmission considering RFID and mmWave systems, ubiquitous tracking with burst and intermittent signals considering WiFi probe requests, and navigation to a Rayleigh fading source. In this chapter, we present the conclusions of this research work and also identify key directions for future research.

In Chapter II, closed-form equations are derived for fundamental limits of localization accuracy for UHF RFID technologies considering realistic radiation patterns. Monostatic and bistatic configurations are compared recognizing tag and reader antenna sensitivities. Our results show that high accuracy of localization does not only depend on the transmit power, but also depends on the use of right elevation angle and antenna placement and the use of bistatic configuration in localization system. In our simulations it is shown that among the considered elevation angles, $\theta=\pi / 2$ yields the best results for the given deployment scenario, since it maximizes the received power, results in largest localization coverage for IoT and minimizes the localization error. We observed that bistatic localization coverage drops with the use of side placement of antennas, while it increases monostatic localization coverage. Using bistatic configurations improves the probability of localizing the tag with higher accuracies when compared with monostatic configurations. The best results are achieved with bistatic configuration and side placement of the antennas. An important direction for future research would be taking Rayleigh fading into consideration for capturing a 
more realistic indoor channel model and studying the feasibility of the RFID system experimentally.

In Chapter III, the fundamental limits of RSS-based mmWave positioning systems studied using beamforming considering indoor scenarios for IEEE 802.11ad SLS. Our results show that high accuracy of localization depends on number of antenna elements. Larger number of antenna elements gives better accuracy. However above a certain number, the localization accuracy saturates due to limitations of beamforming. In our simulations, NLSE diverges from CRLB as number of antenna elements increases since side lobes become an interferer at further distances where number of measurements are limited. Future research directions for this work includes study of NLOS cases with reflections which are more prominent for indoor cases and directional antenna arrays at both ends of the system for a more realistic system scenario.

In Chapter IV, WiFi probe requests are captured at various reference locations for occupancy monitoring in smart buildings and their RSS values are used in positioning and tracking algorithms. The proposed method assures the utilization of the probe requests regardless of the number of the available reference nodes that they are detected, which allows to detect three times more users in the occupancy counting process when compared to requiring at at least four reference nodes for localization. In our experimental results, we are able to perform zone-level occupancy tracking with up to $90 \%$ accuracy. It is possible to determine the peak hours of the individual zones as well as the quiet times of the building. Future work includes use of more advanced filtering techniques such as particle filters to further improve zone level positioning and occupancy counting performance. Another interesting research direction for this study would be application of machine learning techniques at various stages of localization, tracking, and occupancy counting. 
In Chapter V, the problem of navigating a UAV to a Rayleigh fading RF source is considered. The behavior of Q-learning based UAV navigation under Rayleigh fading assumption is studied, and averaging of the RSS over different time spans considering different UAV speeds is investigated. Overall results prove that it is critical to use a Q-learning based approach for avoiding the navigation bias in a Rayleigh fading environment with sufficiently large window. Another observation is that a variable learning rate is preferable compared to a fixed learning rate for increasing responsiveness of the system. Future directions for research about UAV navigation would be using a realistic simulator considering Raytracing models as well as some experimental studies. 


\section{BIBLIOGRAPHY}

[1] N. Patwari and A. O. Hero, III, "Using proximity and quantized RSS for sensor localization in wireless networks," in ACM Int. Conf. Proc. on WSNs and Appl., ser. WSNA '03. NY, USA: ACM, 2003, pp. 20-29.

[2] R. Zekavat and R. M. Buehrer, Handbook of position location: Theory, practice and advances. John Wiley \& Sons, 2011, vol. 27.

[3] J. Figueiras and S. Frattasi, Mobile positioning and tracking: from conventional to cooperative techniques. John Wiley \& Sons, 2011.

[4] Z. Sahinoglu, S. Gezici, and I. Guvenc, "Ultra-wideband positioning systems," Cambridge, New York, 2008.

[5] The Internet of Things Infographic, Cisco. [Online]. Available: http: //blogs.cisco.com/diversity/the-internet-of-things-infographic

[6] V. Erickson, S. Achleitner, and A. Cerpa, "POEM: power-efficient occupancybased energy management system," in Proc. IEEE Information Processing in Sensor Networks (IPSN), Apr 2013, pp. 203-216.

[7] A. Zanella, N. Bui, A. Castellani, L. Vangelista, and M. Zorzi, "Internet of things for smart cities," IEEE Internet of Things J., vol. 1, no. 1, pp. 22-32, Feb. 2014.

[8] J. Jin, J. Gubbi, S. Marusic, and M. Palaniswami, "An information framework for creating a smart city through Internet of things," IEEE Internet of Things J., vol. 1, no. 2, pp. 112-121, Apr. 2014.

[9] P. Vlacheas, R. Giaffreda, V. Stavroulaki, D. Kelaidonis, V. Foteinos, G. Poulios, P. Demestichas, A. Somov, A. Biswas, and K. Moessner, "Enabling smart cities through a cognitive management framework for the Internet of things," IEEE Commun. Mag., vol. 51, no. 6, pp. 102-111, June 2013.

[10] Y. Agarwal, B. Balaji, R. Gupta, J. Lyles, M. Wei, and T. Weng, "Occupancydriven energy management for smart building automation," in Proc. ACM Workshop on Embedded Sensing Systems for Energy-Efficiency in Building, 2010, pp. 1-6.

[11] L. Yan, Y. Zhang, L. Yang, and H. Ning, The Internet of Things: from RFID to the Next-Generation Pervasive Networked Systems, ser. Wireless Networks and Mobile Communications. Taylor \& Francis, 2008. 
[12] E. Welbourne, L. Battle, G. Cole, K. Gould, K. Rector, S. Raymer, M. Balazinska, and G. Borriello, "Building the Internet of Things using RFID: the RFID ecosystem experience," IEEE Internet Computing, vol. 13, no. 3, pp. 48-55, May 2009.

[13] L. Atzori, A. Iera, and G. Morabito, "The Internet of Things: A survey," Computer Networks, vol. 54, no. 15, pp. 2787-2805, Oct. 2010.

[14] I. Guvenc, S. Gezici, Z. Sahinoglu, and U. C. Kozat, Reliable communications for short-range wireless systems. Cambridge University Press, 2011.

[15] K. Akkaya, I. Guvenc, R. Aygun, N. Pala, and A. Kadri, "Iot-based occupancy monitoring techniques for energy-efficient smart buildings," in Wireless Communications and Networking Conference Workshops (WCNCW), 2015 IEEE, March 2015, pp. 58-63.

[16] X. Jia, Q. Feng, T. Fan, and Q. Lei, "RFID technology and its applications in internet of things (IoT)," in Proc. Int. Conf. Consumer Electronics, Communications and Networks (CECNet), Apr. 2012, pp. 1282-1285.

[17] S. Wamba and E. W. Ngai, "Importance of the relative advantage of RFID as enabler of asset management in the healthcare: Results from a delphi study," in Proc. 45th Hawaii Int. Conf. System Science (HICSS), Jan. 2012, pp. 28792889.

[18] M. Rieback, B. Crispo, and A. Tanenbaum, "Keep on blockin in the free world: Personal access control for low-cost RFID tags," in Security Protocols, B. Christianson, B. Crispo, J. Malcolm, and M. Roe, Eds. Springer Berlin Heidelberg, 2007, vol. 4631, pp. 51-59.

[19] T. D. Rty, "Survey on contemporary remote surveillance systems for public safety," IEEE Trans. on Syst., Man, and Cyber., vol. 40, no. 5, pp. 493-515, Sept. 2010.

[20] M. Darianian and M. Michael, "Smart home mobile RFID-based Internet-ofThings systems and services," in Proc. Int. Conf. Advanced Computer Theory and Engineering, Dec. 2008, pp. 116-120.

[21] A. Al-Ali, F. Aloul, N. Aji, A. Al-Zarouni, and N. Fakhro, "Mobile RFID tracking system," in Proc. Int. Conf. Information and Communication Technologies: From Theory to Applications, Apr. 2008, pp. 1-4. 
[22] G. Kortuem, F. Kawsar, D. Fitton, and V. Sundramoorthy, "Smart objects as building blocks for the Internet of Things," IEEE Internet Computing, vol. 14, no. 1, pp. 44-51, Jan. 2010.

[23] N. Patwari, J. Ash, S. Kyperountas, A. Hero, R. Moses, and N. Correal, "Locating the nodes: cooperative localization in wireless sensor networks," IEEE Sig. Proc. Mag., vol. 22, no. 4, pp. 54-69, July 2005.

[24] S. Gezici, Z. Tian, G. Giannakis, H. Kobayashi, A. Molisch, H. Poor, and Z. Sahinoglu, "Localization via ultra-wideband radios: a look at positioning aspects for future sensor networks," IEEE Sig. Proc. Mag., vol. 22, no. 4, pp. 70-84, July 2005.

[25] E. Alimpertis, N. Fasarakis-Hilliard, and A. Bletsas, "Community RF sensing for source localization," IEEE Wirel. Commun. Lett., vol. 3, no. 4, pp. 393-396, 2014.

[26] I. Guvenc, S. Gezici, and Z. Sahinoglu, "Fundamental limits and improved algorithms for linear least-squares wireless position estimation," Wireless Comm. and Mobile Computing, vol. 12, no. 12, pp. 1037-1052, 2012.

[27] M. Merenda, C. Felini, and F. Della Corte, "Battery-less smart RFID tag with sensor capabilities," in Proc. IEEE Int. Conf. RFID-Technologies and Applications (RFID-TA), Nov. 2012, pp. 160-164.

[28] R. Miesen, R. Ebelt, F. Kirsch, T. Schafer, G. Li, H. Wang, and M. Vossiek, "Where is the tag?" IEEE Microwave Mag., vol. 12, no. 7, pp. S49-S63, Dec. 2011.

[29] L. Ni, D. Zhang, and M. Souryal, "RFID-based localization and tracking technologies," IEEE Wireless Comm., vol. 18, no. 2, pp. 45-51, Apr. 2011.

[30] M. Bouet and A. dos Santos, "RFID tags: positioning principles and localization techniques," in Proc. Wireless Days, Nov. 2008, pp. 1-5.

[31] L. Geng, M. Bugallo, A. Athalye, and P. Djuric, "Real time indoor tracking of tagged objects with a network of RFID readers," in Proc. European Signal Processing Conference (EUSIPCO), Aug. 2012, pp. 205-209.

[32] M. Moreno, M. Zamora, J. Santa, and A. Skarmeta, "An indoor localization mechanism based on RFID and IR data in ambient intelligent environments," 
in Proc. Int. Conf. Innovative Mobile and Internet Services in Ubiquitous Computing (IMIS), July 2012, pp. 805-810.

[33] D. Hahnel, W. Burgard, D. Fox, K. Fishkin, and M. Philipose, "Mapping and localization with RFID technology," in Proc. IEEE Int. Conf. Robotics and Automation, vol. 1, Apr. 2004, pp. 1015-1020 Vol.1.

[34] L. Ni, Y. Liu, Y. C. Lau, and A. Patil, "LANDMARC: indoor location sensing using active RFID," in Proc. IEEE Int. Conf. Pervasive Computing and Commun., Mar. 2003, pp. 407-415.

[35] Y. Zhao, Y. Liu, and L. Ni, "VIRE: Active RFID-based localization using virtual reference elimination," in Proc. Int. Conf. Parallel Processing, Sept. 2007, pp. $56-56$.

[36] A. Bekkali, H. Sanson, and M. Matsumoto, "RFID indoor positioning based on probabilistic RFID map and Kalman filtering," in Proc. IEEE Int. Conf. Wireless and Mobile Computing, Oct. 2007, pp. 21-21.

[37] C. Hekimian-Williams, B. Grant, X. Liu, Z. Zhang, and P. Kumar, "Accurate localization of RFID tags using phase difference," in Proc. IEEE Int. Conf. RFID, Apr. 2010, pp. 89-96.

[38] E. Leitinger, P. Meissner, M. Frohle, and K. Witrisal, "Performance bounds for multipath-assisted indoor localization on backscatter channels," in Proc. IEEE Int. Radar Conf., May 2014, pp. 70-75.

[39] B. S. Ciftler, A. Kadri, and I. Guvenc, "Experimental performance evaluation of passive uhf rfid systems under interference," in in Proc. of IEEE Int. Conf. on RFID Tech. and Appl. (RFID-TA), Sept. 2015, pp. 81-86.

[40] X. Zheng, H. Liu, J. Yang, Y. Chen, R. Martin, and X. Li, "A study of localization accuracy using multiple frequencies and powers," IEEE Trans. Parallel and Distributed Syst., vol. 25, no. 8, pp. 1955-1965, Aug. 2014.

[41] J.-M. Akre, X. Zhang, S. Baey, B. Kervella, A. Fladenmuller, M. Zancanaro, and M. Fonseca, "Accurate 2-d localization of RFID tags using antenna transmission power control," in Wireless Days (WD), 2014 IFIP, Nov 2014, pp. 1-6.

[42] D. Lieckfeldt, J. You, and D. Timmermann, "Exploiting RF-scatter: Human localization with bistatic passive UHF RFID-systems," in IEEE Int. Conf. on Wireless and Mobile Computing, Netw. and Comm., Oct. 2009, pp. 179-184. 
[43] M. Hasani, E.-S. Lohan, L. Sydanheimo, and L. Ukkonen, "Path-loss model of embroidered passive RFID tag on human body for indoor positioning applications," in IEEE RFID Tech. and Appl. Conf. (RFID-TA), Sept. 2014, pp. $170-174$.

[44] B. S. Ciftler, A. Kadri, and I. Guvenc, "Fundamental bounds on RSS-based wireless localization in passive UHF RFID systems," in IEEE Wir. Comm. and Netw. Conf. (WCNC), Mar. 2015, pp. 1356-1361.

[45] K. Chawla, C. McFarland, G. Robins, and C. Shope, "Real-time RFID localization using RSS," 2013 Int. Conf. Localization GNSS, ICL-GNSS 2013, pp. $0-5,2013$.

[46] A. Bekkali, S. Zou, A. Kadri, M. Crisp, and R. Penty, "Performance analysis of passive UHF RFID systems under cascaded fading channels and interference effects," IEEE Trans. Wireless Commun., vol. 14, no. 3, pp. 1421-1433, Mar. 2015 .

[47] EPC Radio-Frequency Identity Protocols Class-1 Generation-2 UHF RFID Protocol for Communications at $860 \mathrm{MHz}-960 \mathrm{MHz}$ Version 1.0.9. EPC Global, Tech. Rep., Jan. 2005.

[48] J. Griffin and G. Durgin, "Complete link budgets for backscatter-radio and RFID systems," IEEE Antennas and Propagation Magazine, vol. 51, no. 2, pp. 11-25, Apr. 2009.

[49] P. Nikitin and K. Rao, "Theory and measurement of backscattering from RFID tags," IEEE Antennas and Propagation Magazine, vol. 48, no. 6, pp. 212-218, Dec. 2006.

[50] A. Bletsas, A. Dimitriou, and J. Sahalos, "Improving backscatter radio tag efficiency," IEEE Trans. on Microwave Theory and Techniques, vol. 58, no. 6, pp. 1502-1509, June 2010.

[51] P. Nikitin, K. Rao, and S. Lam, "UHF RFID tag characterization: overview and state-of-the-art," in Antenna Measurement Techniques Association Symposium (AMTA), 2012.

[52] S. M. Kay, Fundamentals of Statistical Signal Processing: Estimation Theory. Upper Saddle River, NJ, USA: Prentice-Hall, Inc., 1993. 
[53] K. Chawla, C. McFarland, G. Robins, and C. Shope, "Real-time RFID localization using RSS," in Int. Conf. on Localization and GNSS (ICL-GNSS), June 2013, pp. 1-6.

[54] T. S. Rappaport, S. Sun, R. Mayzus, H. Zhao, Y. Azar, K. Wang, G. N. Wong, J. K. Schulz, M. Samimi, and F. Gutierrez, "Millimeter wave mobile communications for 5G cellular: It will work!" IEEE Access, vol. 1, pp. 335-349, 2013.

[55] T. S. Rappaport, R. W. Heath Jr, R. C. Daniels, and J. N. Murdock, Millimeter wave wireless communications. Pearson Education, 2014.

[56] T. Nitsche, C. Cordeiro, A. B. Flores, E. W. Knightly, E. Perahia, and J. C. Widmer, "Ieee 802.11ad: directional $60 \mathrm{ghz}$ communication for multi-gigabitper-second wi-fi [invited paper]," IEEE Communications Magazine, vol. 52, no. 12, pp. 132-141, December 2014.

[57] Y. Zhu, Z. Zhang, Z. Marzi, C. Nelson, U. Madhow, B. Y. Zhao, and H. Zheng, "Demystifying 60ghz outdoor picocells," in Proceedings of the 20th Annual International Conference on Mobile Computing and Networking, ser. MobiCom '14. New York, NY, USA: ACM, 2014, pp. 5-16. [Online]. Available: http://doi.acm.org/10.1145/2639108.2639121

[58] S. Kutty and D. Sen, "Beamforming for millimeter wave communications: An inclusive survey," IEEE Communications Surveys Tutorials, vol. 18, no. 2, pp. 949-973, Secondquarter 2016.

[59] F. Lemic, J. Martin, C. Yarp, D. Chan, V. Handziski, R. Brodersen, G. Fettweis, A. Wolisz, and J. Wawrzynek, "Localization as a feature of mmwave communication," in 2016 International Wireless Communications and Mobile Computing Conference (IWCMC), Sept 2016, pp. 1033-1038.

[60] A. Olivier, G. Bielsa, I. Tejado, M. Zorzi, J. Widmer, and P. Casari, "Lightweight indoor localization for 60-ghz millimeter wave systems," in 2016 13th Annual IEEE International Conference on Sensing, Communication, and Networking (SECON), June 2016, pp. 1-9.

[61] K. Witrisal, P. Meissner, E. Leitinger, Y. Shen, C. Gustafson, F. Tufvesson, K. Haneda, D. Dardari, A. F. Molisch, A. Conti, and M. Z. Win, "High-accuracy localization for assisted living: $5 \mathrm{~g}$ systems will turn multipath channels from foe to friend," IEEE Signal Processing Magazine, vol. 33, no. 2, pp. 59-70, March 2016 . 
[62] M. Vari and D. Cassioli, "mmwaves rssi indoor network localization," in 2014 IEEE International Conference on Communications Workshops (ICC), June 2014, pp. 127-132.

[63] F. T. Ulaby, E. Michielssen, and U. Ravaioli, "Fundamentals of applied electromagnetics 6e," Boston, Massachussetts: Prentice Hall, 2010.

[64] K. Pahlavan, P. Krishnamurthy, and Y. Geng, "Localization challenges for the emergence of the smart world," IEEE Access, vol. 3, pp. 3058-3067, 2015.

[65] G. Pan, G. Qi, W. Zhang, S. Li, Z. Wu, and L. Yang, "Trace analysis and mining for smart cities: issues, methods, and applications," IEEE Commun. Mag., vol. 51, no. 6, pp. 120-126, June 2013.

[66] S. Tadokoro, Q. shan Jia, Q. Zhao, H. Darabi, G. Huang, B. Becerik-Gerber, H. Sandberg, and K. Johansson, "Smart building technology," IEEE Robotics Automation Mag., vol. 21, no. 2, pp. 18-20, June 2014.

[67] K. Akkaya, I. Guvenc, R. Aygun, N. Pala, and A. Kadri, "IoT-based occupancy monitoring techniques for energy-efficient smart buildings," in Proc. IEEE Wireless Commun. Networking Conference Workshops (WCNCW), Mar. 2015, pp. 58-63.

[68] B. Balaji, J. Xu, A. Nwokafor, R. Gupta, and Y. Agarwal, "Sentinel: Occupancy based HVAC actuation using existing WiFi infrastructure within commercial buildings," in Proc. ACM Conf. on Embedded Networked Sensor Systems, 2013, pp. 17:1-17:14. [Online]. Available: http://doi.acm.org/10. $1145 / 2517351.2517370$

[69] E. Vattapparamban, B. S. Ciftler, I. Guvenc, K. Akkaya, and A. Kadri, "Indoor occupancy tracking in smart buildings using passive sniffing of probe requests," in Proc. IEEE International Conference on Communications Workshops (ICC), May 2016, pp. 38-44.

[70] L. Demir, "Wi-Fi tracking: what about privacy," Ph.D. dissertation, M2 SCCI Security, Cryptologyand Coding of Information-UFR IMAG, 2013.

[71] J. Freudiger, "How talkative is your mobile device?: An experimental study of Wi-Fi probe requests," in Proc. ACM Conf. Security and Privacy in Wireless and Mobile Networks, New York, NY, USA, 2015, pp. 8:1-8:6. [Online]. Available: http://doi.acm.org/10.1145/2766498.2766517 
[72] A. B. M. Musa and J. Eriksson, "Tracking unmodified smartphones using Wi-fi monitors," in Proc. ACM Conf. Embedded Network Sensor Systems, ser. SenSys '12. New York, NY, USA: ACM, 2012, pp. 281-294. [Online]. Available: http://doi.acm.org/10.1145/2426656.2426685

[73] D. Namiot and M. Sneps-Sneppe, "On the analysis of statistics of mobile visitors," Automatic Control and Computer Sciences, vol. 48, no. 3, pp. 150-158, 2014 .

[74] Hak5. (2013) Wi-Fi Pineapple Mark V. [Online]. Available: http: //hakshop.myshopify.com/products/wifi-pineapple

[75] J. Ryou, B. Lee, C. Yu, M. Kim, S.-J. Hyun, S.-M. Park, and W.-T. Kim, "Probe request based load balancing metric with timely handoffs for wlans," in Proc. IEEE Int. Conf. Inf. Commun. Tech. Convergence (ICTC), Nov 2010, pp. 346-351.

[76] A. Mishra, M. Shin, and W. Arbaugh, "An empirical analysis of the ieee 802.11 mac layer handoff process," SIGCOMM Comput. Commun. Rev., vol. 33, no. 2, pp. 93-102, Apr. 2003. [Online]. Available: http://doi.acm.org/10.1145/956981.956990

[77] J. Millikenl, V. Selis, K. M. Yap, and A. Marshall, "The effect of probe interval estimation on attack detection performance of a WLAN independent intrusion detection system," in Proc. IET Int. Conf. Wireless Commun. Appl. (ICWCA), Oct 2012, pp. 1-6.

[78] M. Cunche, M. A. Kaafar, and R. Boreli, "I know who you will meet this evening! linking wireless devices using Wi-Fi probe requests," in Proc. IEEE Int. Symp. World of Wireless, Mobile and Multimedia Networks (WoWMoM), June 2012, pp. 1-9.

[79] F. Zampella, A. R. J. Ruiz, and F. S. Granja, "Indoor positioning using efficient map matching, RSS measurements, and an improved motion model," IEEE Trans. Veh. Technol., vol. 64, no. 4, pp. 1304-1317, April 2015.

[80] D. Dardari, P. Closas, and P. M. Djuri, "Indoor tracking: Theory, methods, and technologies," IEEE Trans. Veh. Technol., vol. 64, no. 4, pp. 1263-1278, April 2015. 
[81] J. Castro-Arvizu, J. Vila-Valls, P. Closas, and J. Fernandez-Rubio, "Simultaneous tracking and rss model calibration by robust filtering," in Proc. of Asilomar Conf. on Signals, Syst. and Comp., Nov. 2014, pp. 706-710.

[82] Y. Guo, K. Huang, N. Jiang, X. Guo, Y. Li, and G. Wang, "An exponentialrayleigh model for rss-based device-free localization and tracking," Mobile Computing, IEEE Transactions on, vol. 14, no. 3, pp. 484-494, March 2015.

[83] Y. Kim, H. Shin, and H. Cha, "Smartphone-based wi-fi pedestrian-tracking system tolerating the rss variance problem," in Pervasive Computing and Communications (PerCom), 2012 IEEE International Conference on, March 2012, pp. 11-19.

[84] A. W. S. Au, C. Feng, S. Valaee, S. Reyes, S. Sorour, S. N. Markowitz, D. Gold, K. Gordon, and M. Eizenman, "Indoor tracking and navigation using received signal strength and compressive sensing on a mobile device," IEEE Trans. Mobile Comp., vol. 12, no. 10, pp. 2050-2062, Oct 2013.

[85] M. R. Gholami, S. Gezici, and E. G. Strom, "Improved position estimation using hybrid TW-TOA and TDOA in cooperative networks," IEEE Trans. Sig. Processing, vol. 60, no. 7, pp. 3770-3785, July 2012.

[86] B. J. Dil and P. J. M. Havinga, "RSS-based self-adaptive localization in dynamic environments," in Proc. IEEE Int. Conf. Internet of Things, Oct. 2012, pp. 5562.

[87] A. Bose and C. H. Foh, "A practical path loss model for indoor WiFi positioning enhancement," in Proc. 6th International Conference on Information, Communications Signal Processing, Dec 2007, pp. 1-5.

[88] I. Guvenc and C.-C. Chong, "A survey on TOA based wireless localization and NLOS mitigation techniques," IEEE Commun. Surveys Tuts., vol. 11, no. 3, 2009 .

[89] M. R. Gholami, R. M. Vaghefi, and E. G. Strm, "Rss-based sensor localization in the presence of unknown channel parameters," IEEE Transactions on Signal Processing, vol. 61, no. 15, pp. 3752-3759, Aug 2013.

[90] I. Guvenc, C. T. Abdallah, R. Jordan, and O. Dedeoglu, "Enhancements to RSS based indoor tracking systems using Kalman filters," in Proc. GSPx 83 International Signal Processing Conference, 2003, pp. 91-102. 
[91] J. Yim, C. Park, J. Joo, and S. Jeong, "Extended Kalman filter for wireless LAN based indoor positioning," Decision support systems, vol. 45, no. 4, pp. 960-971, 2008.

[92] J. Hartikainen, A. Solin, and S. Särkkä, "Optimal filtering with Kalman filters and smoothers," Department of Biomedica Engineering and Computational Sciences, Aalto University School of Science: Greater Helsinki, Finland, vol. 16, 2011.

[93] L. Demir, "Wi-Fi tracking: what about privacy," MS Thesis, 2013. [Online]. Available: https://hal.inria.fr/hal-00859013

[94] N. H. Motlagh, T. Taleb, and O. Arouk, "Low-altitude unmanned aerial vehicles-based internet of things services: Comprehensive survey and future perspectives," IEEE Internet of Things Journal, vol. 3, no. 6, pp. 899-922, 2016.

[95] C. Du and S. Zhu, "Research on urban public safety emergency management early warning system based on technologies for the internet of things," Procedia Engineering, vol. 45, pp. 748-754, 2012.

[96] E. Vattapparamban, B. S. Çiftler, İ. Güvenç, K. Akkaya, and A. Kadri, "Indoor occupancy tracking in smart buildings using passive sniffing of probe requests," in Communications Workshops (ICC), 2016 IEEE International Conference on. IEEE, 2016, pp. 38-44.

[97] F. Koohifar, A. Kumbhar, and I. Guvenc, "Receding horizon multi-uav cooperative tracking of moving rf source," IEEE Communications Letters, 2016.

[98] W. Wang, R. Joshi, A. Kulkarni, W. K. Leong, and B. Leong, "Feasibility study of mobile phone wifi detection in aerial search and rescue operations," in Proceedings of the 4th Asia-Pacific Workshop on Systems. ACM, 2013, p. 7.

[99] N. Atanasov, J. Le Ny, N. Michael, and G. J. Pappas, "Stochastic source seeking in complex environments," in Robotics and Automation (ICRA), 2012 IEEE International Conference on. IEEE, 2012, pp. 3013-3018.

[100] J. Fink and V. Kumar, "Online methods for radio signal mapping with mobile robots," in Robotics and Automation (ICRA), 2010 IEEE International Conference on. IEEE, 2010, pp. 1940-1945. 
[101] T. Hester and P. Stone, "Texplore: real-time sample-efficient reinforcement learning for robots," Machine learning, vol. 90, no. 3, pp. 385-429, 2013.

[102] E. U. T. R. Access, "Further advancements for e-utra physical layer aspects (rel. 9), 3rd generation partnership project (3gpp)," Sophia-Antipolis, France, vol. 13, 2010.

[103] A. Konar, I. G. Chakraborty, S. J. Singh, L. C. Jain, and A. K. Nagar, "A deterministic improved q-learning for path planning of a mobile robot," IEEE Transactions on Systems, Man, and Cybernetics: Systems, vol. 43, no. 5, pp. 1141-1153, 2013.

[104] C. J. Watkins and P. Dayan, "Q-learning," Machine learning, vol. 8, no. 3-4, pp. 279-292, 1992.

[105] C. Chen, D. Dong, H.-X. Li, J. Chu, and T.-J. Tarn, "Fidelity-based probabilistic q-learning for control of quantum systems," IEEE transactions on neural networks and learning systems, vol. 25, no. 5, pp. 920-933, 2014. 
VITA

\section{BEKIR SAIT CIFTLER}

October 16, 1989

2011

2013
Born, Iskenderun, TURKEY

B.Sc., Electrical

Middle East Technical University

Ankara, TURKEY

M.Sc., Electrical

TOBB University of Economics and Technology Ankara, TURKEY

\section{PUBLICATIONS AND PRESENTATIONS}

J1) H.U. Yildiz, B.S. Ciftler, B. Tavli, K. Bicakci, and D. Incebacak, "The Impact of Incomplete Secure Connectivity on the Lifetime of Wireless Sensor Networks" published in IEEE Systems Journal (Feb. 2016).

J2) B.S. Ciftler, A. Kadri, and I. Guvenc, "IoT Localization for Bistatic Passive UHF RFID Systems with 3D Radiation Pattern" to be published in IEEE Internet of Things Journal (Accepted: Apr. 2017).

J3) B.S. Ciftler, S. Dikmese, K. Akkaya, A. Kadri, and I. Guvenc, "Indoor Occupancy Tracking based on passive sniffing of burst WiFi probe requests" in review for IEEE Internet of Things Journal Special Issue on Smart Cities (Submitted Jan. 2017).

J4) B.S. Ciftler, I. Guvenc, and K. Akkaya "A survey on IoT-based Occupancy Monitoring" in preparation for IEEE Communications Surveys and Tutorials (Planned June 2017).

J5) Z. Sahinoglu, B.S. Ciftler, and I. Guvenc, "IoT-Based Device-free Occupancy Detection for Energy Efficiency" in review for IEEE Communications Magazine Featured Topic on Internet of Things and Information Processing in Smart Energy Applications (Submitted in Mar. 2017).

L1) B.S. Ciftler, A. Tuncer, and I. Guvenc, "Indoor UAV Navigation to a Rayleigh Fading Source Using Q-Learning" in review for IEEE Internet of Things Journal (Submitted: Nov. 2016)

L2) B.S. Ciftler, N. Rupasinghe, M. Ruble, and I. Guvenc, "mmWave Positioning and Tracking" in preparation for an IEEE Journal (Planned Apr. 2017). 
C1) K. Vasudeva, B.S. Ciftler, A. Altamar and I. Guvenc, "An Experimental Study on RSS Based Wireless Localization with Software Defined Radio," in Proc. of IEEE Wireless and Microwave Technology Conf. (WAMICON), June 2014.

C2) B.S. Ciftler, A. Kadri, and I. Guvenc. "Fundamental bounds on RSS-based wireless localization in passive UHF RFID systems," in Proc. of IEEE Wireless Communications and Networking Conference (WCNC), Mar. 2015.

C3) P. Armengol, R. Tobkes, K. Akkaya, B.S. Ciftler and I. Guvenc, "Efficient Privacy Preserving Fingerprint-based Indoor Localization Using Crowdsourcing," presented at the National Workshop for REU Research in Netw. and Syst. (REUNS), Oct. 2015.

C4) B. S. Ciftler, A. Kadri, and I. Guvenc, "Experimental performance evaluation of passive UHF RFID systems under interference," in Proc. of IEEE RFID Technology and Applications (RFID-TA), Sept. 2015.

C5) E. Vattapparamban, B.S. Ciftler, I. Guvenc, K. Akkaya, and A. Kadri, "Indoor Occupancy Tracking in Smart Buildings Using Passive Sniffing of Probe Requests," in Proc. of IEEE ICC 2016 Workshop on Advances in Network Localization and Navigation (ANLN), May 2016. 